\title{
An annotated checklist of fishes of the family Sciaenidae
}

\author{
Paolo Parenti \\ Department of Earth and Environmental Sciences, University of Milano-Bicocca Piazza della Scienza, 1, \\ 20126 Milano, Italy \\ Corresponding author ${ }^{凶}$ :paolo.parenti@unimib.it
}

Received: 17 January 2020 Accepted: 8 March 2020 Published online: 31 March 2020

\begin{abstract}
A checklist of the croakers of the world, family Sciaenidae, is presented. A total of 584 nominal species belonging to 289 valid species and 69 genera is included. Four genera, Johnius with 32 species, Cynoscion 25, Stellifer 24, and Umbrina 17 contains $30 \%$ of the species, whereas $43 \%$ of the genera (31) are monotypic. Eques is a valid genus-name and should be used instead of Equetus. Fourteen nominal species remain unidentifiable and are placed in incertae sedis, whereas 12 nominal species currently described in sciaenid genera lay outside the family. Among this latter group two of them represent senior synonyms of wellestablished species: Sciaena guttata Bloch and Schneider, 1801 and Sciaena pallida Walbaum, 1792 predate Giuris margaritaceus (Valenciennes, 1837) and Cymolutes praetextatus (Quoy and Gaimard, 1824), respectively, and they are here declared nomina oblita.
\end{abstract}

Key words: Croakers, Eques, nomina oblita, nominal species

\section{Introduction}

Sciaenidae are marine, brackish and freshwater fishes. They can be diagnosed as follows: body oblong to slightly elongate, compressed and some with moderately high body (Eques, Pogonias, Cheilotrema); head and body scaled except the tip of the snout. Scales cycloid or ctenoid covering the soft dorsal, anal and caudal fins; lateral line to end of caudal fin and with pored scales often with intercalated small scales, which make the lateral line appear much thicker. Two nostrils; mouth variable from strongly inferior to oblique with small, sharp, and conical teeth in rows on jaws; enlarged teeth always form outer series in upper jaw, inner series in lower jaw; well-developed canines may be present at front of upper jaw (Cynoscion) or both jaws (Macrodon); vomer and palatine toothless; gill membranes free from isthmus. Barbel on the chin present or absent. Otoliths exceptionally large. Skull cavernous with a series of bridge-like bony struts; infraorbitals and preopercle with variously developed ridges which fold over canals of cephalic lateral-line system. A series of sensory pores are present at the tip of the snout (3-7 rostral pores), on the lower margin of the snout (2-5 marginal pores) and on the tip of the chin (2-6 mental pores); chin with a single barbel (Menticirrhus), or in pairs along median edges of lower jaw (Micropogonias) or subopercles (Paralonchurus, Pogonias). Branchiostegal rays 7. Dorsal fin continuous with VII-XIV spines and 18-46 soft rays, deeply notched before the last spine; anal fin with II spines (very strong in Bairdiella; one spine in Menticirrhus americanus), 6-9 soft rays; pectoral fin with 6-20 rays; pelvic fin I, 5. Caudal fin emarginated to pointed in juveniles, rounded to rhomboid in adult, with 15 
branched rays. The swim bladder is thick walled, typically occupies the abdominal cavity and varies from simple (e.g. Sciaena trewavasae) or two chambered without appendages (e.g., Corvula macrops) to divided into an anterior and posterior chamber with or without bud-like, horn-like, tube-like or arborescent appendages. The drumming muscle is typically found in males and weak or absent in females. The coloration varies from silvery to yellowish or dark brown; dark spots, vertical bars and longitudinal stripes are often present; tip of spinous dorsal fin is dark edged in many species; abdominal and lower fins are yellowish in many instances; a dark blotch is often present at pectoral-fin bases; roof of mouth and lining of gill cavity is often black.

Their common name derives from their ability to produce drumming or croaking sounds through specialized body muscles connected to the swim bladder, which acts as a resonating chamber. The sound production could play a role during spawning season. Widespread in the Atlantic, Indian and Pacific oceans, they are generally bottom dwellers that inhabit sandy and muddy areas of near continental region, frequently off beaches, estuaries, sheltered bays and mouths of rivers (Nelson et al., 2016).

Others occur offshore at greater depth (Protosciaena bathytatos to $600 \mathrm{~m}$ ), whereas a number are strictly freshwater, particularly in South America (Pachypops, Pachyurus, Plagioscion). Others inhabit coral reefs (Eques). Members of the Sciaenidae are absent from the islands of the central Indian and Pacific oceans. They are generally voracious carnivores feeding on small fishes and benthic invertebrates. Croakers represent an important component of nearshore bottom trawl catches. Nearly all are excellent food. The swim bladders of Cynoscion are used in the industrial production of isinglass.

\section{Material and Methods}

Genera and species are listed alphabetically together with synonyms, type localities, type material and distribution. Authorship, date of publication, and details of subsequent designation of type species of the genera are given by Fricke et al. (2019). Misidentifications are included only when a later author, upon noting that a misidentification is a new species, named it for the author who made the misidentification. The list of institutions where the type material is located is in Appendix.

\section{Results and Discussion}

Trewavas (1962) revised the West African species of sciaenids and Trewavas (1977) also revised the Indo-West Pacific species, while Chao (1978) reviewed the western Atlantic species. A key to the eastern Pacific species, representing about one third of the world Sciaenidae, has been prepared first by McPhail (1958) and later by Chao (1995). The FAO species identification guides cover the sciaenid species because of their interest to fisheries of the major marine resource groups exploited in the Western Central Pacific (Sasaki, 2001), Western Indian Ocean (Lal Mohan, 1983), Eastern Central Atlantic (Chao, 2016) and Western Central Atlantic (Chao, 2002b).

About 265 species distributed in 10 subfamilies are reported in the revision of the family prepared by Sasaki (1989) while 67 genera with 283 species are recorded in a more recent account by Nelson et al. (2016). Allen and Robertson (1994) following the opinion of Chao (pers. comm. in Bauchot and Desoutter, 1987) estimate there are 80 genera with about 300 species. After recent contributions (Sasaki, 1995b, 1996, 1997, 1999; Casatti, 2002b; Iwatsuki et al., 2012; Azpelicueta et al., 2019; Chao et al., 2019; Marceniuk et al., 2019) there are 289 species in 69 genera. 
In the present checklist, information about the status of 584 nominal species is included. Thirty percent of the sciaenid species are included in four genera (Johnius with 32 species, Cynoscion 25, Stellifer 24, and Umbrina 17) whereas $43 \%$ of the genera (31) are monotypic. Eques is a valid genus-name and should be used instead of Equetus. Two nominal species represent senior synonyms of well-established species: Sciaena guttata Bloch and Schneider, 1801 is a senior synonym of the eleotrid Giuris margaritaceus (Valenciennes, 1837) and Sciaena pallida Walbaum, 1792 predates Cymolutes praetextatus (Quoy and Gaimard, 1824). Sciaena guttata and Sciaena pallida are here declared nomina oblita.

\section{Species accounts}

Class Actinopterygii Cope, 1887

Order Perciformes Bleeker, 1863

Family Sciaenidae Cuvier, 1829

(Croakers; drummers; kobs)

Scienoides Cuvier, 1829: 171 (proposed as family). Type genus Sciaena. Family name latinized to Sciaenidae by Bonaparte, 1831: 156, 170.

\section{Genus Aplodinotus Rafinesque, 1819}

Aplodinotus Rafinesque, 1819: 418. Masculine. Type species Aplodinotus grunniens Rafinesque, 1819. Type by original designation.

Haploidonotus Gill, 1861d: 101 (unjustified emendation).

Eutychelithus Jordan, 1876: 242 Masculine. Corvina richardsonii Cuvier, 1830. Type by monotypy.

Freshwaters of North and Central America on bottoms of medium to large river and lakes. Monotypic.

\section{Aplodinotus grunniens Rafinesque, 1819}

Aplodinotus grunniens Rafinesque, 1819: 419 (Ohio River, U.S.A.). No types known.

Sciaena grisea Lesueur, 1822: 254 (Ohio River, U.S.A.). Syntypes: (2) whereabouts unknown.

Sciaena oscula Lesueur, 1822: 252, unnumbered plate (apparently 13) (Lake Erie or Lake Ontario, North America). Syntypes: MNHN A-5696 (1, dry, poor condition), A-7536 (lost).

Corvina richardsonii Cuvier in Cuvier and Valenciennes, 1830: 100 (Lake Huron, North America). Holotype (unique): not found at MNHN.

Amblodon concinnus Agassiz, 1854: 307 (Tennessee River at Huntsville, Alabama, U.S.A.). Lectotype: MCZ 21970, selected by Chao (1978: 42).

Amblodon lineatus Agassiz, 1854: 307 (Osage R., Missouri, U.S.A.). Syntypes: MCZ 569 (2), 569a (5), 573 (1); SMNS 1198 (2).

Amblodon neglectus Girard, 1858b: 167 (Mouth of Rio Grande del Norte, Rio Bravo, Texas, U.S.A., and Province of Tamaulipas, Mexico). Syntypes: UMMZ 160256 (1); USNM 636 (1), 637 (orig. 24, now 12).

Distribution: East of Rocky Mountains from Hudson Bay to Mississippi river basin southward to eastern Mexico and Guatemala.

\section{Genus Argyrosomus De la Pylaie, 1835}

Argyrosomus De la Pylaie, 1835: 532 Masculine. Type species Argyrosomus procerus De la Pylaie, 1835 (= Cheilodipterus aquila Lacepède, 1803). Type by monotypy. On Official List (Opinion 988).

Pseudosciaena Bleeker, 1863a: 142. Feminine. Type species Pseudosciaena amblyceps Bleeker, 1863. Type by subsequent designation. Bleeker, 1874: 18 and 1876: 329 listed Sciaena aquila Risso = Lacepède, 1803 as type (accepted by Trewavas, 1977: 387), but 
aquila was not a named included species. Type apparently validly designated first by Jordan, 1919: 321.

Afroscion Trewavas, 1977: 337. Masculine. Type species Argyrosomus thorpei Smith, 1977. Type by original designation.

Eastern Atlantic and Indo-West Pacific, generally nearshore on hard and soft bottoms and in estuaries. Seven species according to Sasaki (1994); the species from Africa and Madagascar have been reviewed by Griffiths and Heemstra (1995); 9 species are listed here.

\section{Argyrosomus amoyensis (Bleeker, 1863)}

Pseudosciaena amoyensis Bleeker, 1863a: 144 (Amoy, China). Holotype (unique): RMNH 740.

Sciaena bleekeri Day, 1876: 185, Pl. 45 (fig. 4) (Mumbai and Gwadur, India). Syntypes: ZSI F968 (1), 988 (1, figured).

Pseudosciaena indica Tang, 1937: 64 (Mumbai and Gwadur, India). Syntypes: ZSI F968 and 988 (2). Nomen novum, replacement name for Sciaena bleekeri Day 1876, secondarily preoccupied by Pseudotolithus bleekeri Steindachner 1866.

Nibea miichthioides Chu, Lo and $\mathrm{Wu}, 1963$, figs. 30, 56, 82 (Cha-po, Kwangtung Province, China). Holotype: SFC 57-0337. Paratypes: SFC 60-9080 (1).

Distribution: Persian Gulf, also entering Tigris-Euphrates basin, to China.

\section{Argyrosomus beccus Sasaki, 1994}

Argyrosomus beccus Sasaki, 1994: 35, figs. 1-2 (Harbor Channel Islands, sand bars off the Channel, now disappeared, 1.6 kilometers west of Harbor Inlet, Durban, South Africa). Holotype: USNM 325504. Paratypes: USNM 325505 (2).

Distribution: Southern Africa.

\section{Argyrosomus coronus Griffiths and Heemstra, 1995}

Sciaena aquila Poll (nec Lacepède), 1954: 234, fig.71, Pl. II (figs. 2, 6-7) (off Angola).

Argyrosomus coronus Griffiths and Heemstra, 1995: 28, figs. 35-36 (4 kilometers south of Hoarusib River, northern Namibia, surf zone). Holotype: SAIAB formerly RUSI 48468. Paratypes: IRSNB 9723-25 (2, 1, 1); SAIAB formerly RUSI 48458-67 (1 ea.), 48469-72 (1 ea.), 48473 (1, skeleton).

Distribution: Namibia and Angola.

\section{Argyrosomus heinii (Steindachner, 1902)}

Sciaena heinii Steindachner, 1902: 317 (Quishn [Gischen], se. Arabia). Holotype (unique): NMW. Illustrated and described in detail in Steindachner 1903: 141, Pl. 1 (fig. 4).

Distribution: Persian Gulf, Pakistan.

\section{Argyrosomus hololepidotus (Lacepède, 1801)}

Labrus hololepidotus Lacepède, 1801: 448, 518, Pl. 21 (fig. 2) (Grand Océan équatorial). Based on a drawing and description in manuscript by Philibert Commerson (1727-1773) from Madagascar. Neotype: SAIAB formerly RUSI 41433 designated by Griffiths and Heemstra, 1995: 17.

Distribution: Madagascar.

\section{Argyrosomus inodorus Griffiths and Heemstra, 1995}

Sciaena hololepidota Cuvier (nec Lacepède) in Cuvier and Valenciennes, 1830: 53 (Cape of Good Hope).

Argyrosomus inodorus Griffiths and Heemstra, 1995: 21, figs. 21-22 (False Bay, South Africa, depth 10 meters). Holotype: SAIAB formerly RUSI 44705. Paratypes: AMS I.34965001 (2), I.34965-002 (2); BMNH 1994.11.30.2-3 (1, 1); MNHN 1994-0568 to 0569 (1, 1); NSMT-P 46548 (1) 56549 (parts); SAIAB formerly RUSI 11924 (1), 15257 (1), 38464 (1), 38466-67 (1, 1), 38469 (1), 38471-72 (1, 1), 38475-76 (1, 1), 38478-81 (1 ea.), 41128 (1), 
41133 (1), 41138 (1), 41141 (1), 41143 (1), $41146-47$ (1, 1), 41149 (1), 41153 (1), 41155-56 (1, 1), 41160-61 (1, 1), 41164 (1); SAM 33473-74 (1, 1); USNM 331636 (2).

Distribution: Namibia to Cape of Good Hope northward to Key River mouth (Transkei).

\section{Argyrosomus japonicas (Temminck and Schlegel, 1843)}

Sciaena japonica Temminck and Schlegel, 1843: 58, Pl. 24 (fig. 1) (Meitsu, Nango-Cho, Miyazaki Pref., se. coast of Kyushu, Japan, 50 m [orig. loc. Near Nagasaki, Japan]). Neotype: SAIAB formerly RUSI 44704. Based on a figure and description of Burger (Boeseman, 1947: 62). Neotype designated by Griffiths and Heemstra 1995: 11.

Sciaena antarctica Castelnau, 1872: 100, Pl. 1 (Melbourne market, Bass Strait, Australia). No types known.

Sciaena margaritifera Haly, 1875: 269 (off Kwa Zulu-Natal, South Africa, southwestern Indian Ocean). Syntypes: BMNH 1862.11.9.13-14 (2).

Corvina axillaris De Vis, 1884: 538 (Brisbane River, Queensland, Australia). Objectively invalid, nomen praeoccupatum, preoccupied by Corvina axillaris Cuvier, 1830.

Sciaena neglecta Ramsay and Ogilby, 1887: 941 (Broken Bay, New South Wales, Australia). Holotype: AMS I.822.

Distribution: Cape of Good Hope to southern Mozambique; southern seabord of Australia from north of Shark Bay to Queensland; Pakistan and northwest coast of India; Hong Kong to southern Korea and Japan.

\section{Argyrosomus regius (Asso, 1801)}

Sciaena umbra var. Brünnich, 1768: 99 (Adriatic Sea).

Perca luth Walbaum, 1792: 334 (In Mari rubro). No types known.

Perca regia Asso, 1801: 42, Pl. 35 (fig. 3) (La Rochelle, Département de la CharenteMaritime, France, Bay of Biscay, Northeastern Atlantic). Neotype: MNHN 0000-7511, designated by Trewavas, 1966: 4 .

Cheilodipterus aquila Lacepède, 1803: 684, 685, Pl. 21 (La Rochelle, France, Bay of Biscay, northeastern Atlantic). Neotype: MNHN 0000-7511.

Perca vanloo Risso, 1810: 298, Pl. 9 (fig. 30) (Nice, France, northwestern Mediterranean Sea). No types known.

Trachurus aguilus Rafinesque, 1810: 42, Pl. 11 (fig. 3) (Sicily, Italy). No type known.

Seriola rafinesquii Risso, 1827: 425 (Mediterranean Sea). Nomen novum, unneeded replacement name for Trachurus aguilus Rafinesque 1810.

Argyrosomus procerus De la Pylaie, 1835: 532 (France). No types known.

Distribution: Eastern Atlantic from southern Norway and Sweden to the Mediterranean and Black Sea; Gulf of Guinea; migrated to the Red Sea via the Suez Canal.

Comments: Perca luth Walbaum, 1792 is nomen oblitum not used as valid after 1899; therefore, reversal of precedence apply (Parenti, 2013). The type locality is erroneous as description is based on Perca (luth) of Hasselquist, 1762: 402 who reported Damiatae as type locality (=Damietta or Domyāt in Arabic, a city of Egypt on Mediterranean Sea. Trachurus aguilus Rafinesque, 1810 is a synonym according to Parenti (2019b).

\section{Argyrosomus thorpei Smith, 1977}

Argyrosomusthorpei Smith, 1977: 562, Pls. 1 (fig. C), 2 (Off Kwa Zulu-Natal, South Africa, southwestern Indian Ocean). Holotype: SAIAB formerly RUSI 674. Paratypes: BMNH 1976.10.18.1 (10); RUSI 675-680 (1, 1, 1, 1, 1, 1), 2587 (1), 2651 (2).

Distribution: Port Elizabeth (southern Africa) to Mozambique and west coast of Madagascar.

\section{Genus Aspericorvina Fowler, 1934}

Aspericorvina Fowler, 1934: 153. Feminine. Subgenus of Johnius, type species Johnius melanobrachium Fowler, 1934. Type by original designation.

West Pacific; monotypic. 


\section{Aspericorvina jubata (Bleeker, 1855)}

Corvina jubata Bleeker, 1855b: 160 (Bandjarmasin, Borneo, Indonesia) Holotype (unique): whereabouts unknown. Bleeker specimens: BMNH 1880.4.21.150 (1).

Johnius (Aspericorvina) melanobrachium Fowler, 1934: 154, fig. 122; Bangkok, Thailand. Holotype (unique): ANSP 59956.

Distribution: Indo-Australian Archipelago shallow coastal waters, estuaries and rivers.

\section{Genus Atractoscion Gill, 1862}

Atractoscion Gill, 1862: 18. Masculine. Type species Otolithus aequidens Cuvier 1830. Type by original designation.

Zeluco Whitley, 1931: 317. Masculine. Type species Otolithus atelodus Günther 1867. Type by original designation.

Atlantic, Indo-West Pacific and eastern Pacific. Found in coastal waters, often in schools over rocky bottom and in kelp beds or on sandy bottoms. Five species.

\section{Atractoscion aequidens (Cuvier, 1830)}

Otolithus aequidens Cuvier in Cuvier and Valenciennes, 1830: 66 (Cape of Good Hope, South Africa). Syntypes: MNHN A-5586 (1, dry), A-8154 (1, dry, not found).

Distribution: South Africa and Mozambique (following Song et al., 2017).

\section{Atractoscion atelodus (Günther, 1867)}

Otolithusatelodus Günther, 1867b: 60 (Australia). Holotype (unique): BMNH 1866.6.19.9.

Otolithus teraglin MacLeay, 1880: 48 (Sydney market, N.S.W., Australia). Holotype: AMS I.9773.

Distribution: Endemic to south-eastern coast of Australia.

\section{Atractoscion macrolepis Song, Kim and Kim, 2017}

Atractoscion macrolepis Song, Kim and Kim, 2017 in Song, Kim, Kang and Kim, 2017: 228, fig. 2B (Tombua Beach, Angola, $15.7795^{\circ} \mathrm{S}, 11.8703^{\circ} \mathrm{E}$ ). Holotype: SAIAB 187117-2. Paratypes: SAIAB 205157-1, 187117-6, 187117-7.

Distribution: Angola and Namibia.

\section{Atractoscion microlepis Song, Kim and Kang, 2017}

Atractoscion microlepis Song, Kim and Kang in Song, Kim, Kang and Kim, 2017: 231, fig. 2C (Gulf of Oman). Holotype: PKU 9315. Paratypes: PKU 9312-9314, 9316, PKU1077410783.

Distribution: Gulf of Oman, Arabian Sea.

\section{Atractoscion nobilis (Ayres, 1860)}

Johnius nobilis Ayres, 1860: 77, fig. 20 (San Francisco Bay, California, U.S.A.; Baja California, $27^{\circ} \mathrm{N}$, Mexico). No types known. Any types at CAS would have been lost in 1906.

Otolithus californiensis Steindachner, 1876a: 59 (San Diego, California; Magdalena Bay, Baja California). Syntypes: (several) MCZ 10898 (1), NMW 31128-29 (2).

Distribution: Alaska to southern Baja California and northern Gulf of California.

\section{Genus Atrobucca Chu, Lo and Wu, 1963}

Atrobucca Chu, Lo and Wu, 1963: 64, 93. Feminine. Type species Sciaena nibe Jordan and Thompson, 1911. Type by original designation.

Indo-West Pacific. Ten species.

Atrobucca adusta Sasaki and Kailola, 1988

Atrobucca adusta Sasaki and Kailola, 1988: 270, figs. 2C, 6, 7 (Mouth of Markham River, near Lae, Papua New Guinea). Holotype: QM I.19180. Paratype: KFRS F03381. 
Distribution: New Guinea. Known only from two specimens.

Atrobucca alcocki Talwar, 1980

Atrobucca alcocki Talwar, 1980: 23, fig. 1 (Arabian Sea off Mumbai, India, depth about 60 meters). Holotype (unique): ZSI F7591/2.

Distribution: Gulf of Oman, Arabian Sea off Bombay, India and Sind, Pakistan.

\section{Atrobucca antonbruun Sasaki, 1995}

Atrobucca antonbruun Sasaki, 1995b: 269, figs. 1, 3-5 (Off Myanmar, 1504' N, 9551' E, depth 41-46 meters). Holotype: USNM 324670. Paratypes: USNM 324158 (2), 324576 (6), 325470 (2).

Distribution: Burma to east coast of India.

\section{Atrobucca bengalensis Sasaki, 1995}

Atrobucca bengalensis Sasaki, 1995b: 272, figs. 2-5 (St. John's fish market, Trincomalea, Sri Lanka). Holotype: USNM 32463. Paratypes: USNM 325473 (2).

Distribution: Sri Lanka.

\section{Atrobucca brevis Sasaki and Kailola, 1988}

Atrobucca brevis Sasaki and Kailola, 1988: 268, figs. 2B, 4, 5 (Off northern Australia, 13 ${ }^{\circ} 25^{\prime}$ S, $126^{\circ} 19^{\prime}$ E, depth 60-63 meters). Holotype: AMS I. 26447-001. Paratypes: AMS I.26448001 (1); CSIRO H 748-01 (1); HUMZ 107483-84 (1, 1); KFRS F04034 (1).

Distribution: Northern Australia and New Guinea.

\section{Atrobucca geniae Ben-Tuvia and Trewavas, 1987}

Atrobucca geniae Ben-Tuvia and Trewavas, 1987: 17, figs. 1-2 (Gulf of Aqaba, Red Sea, depth 750 meters). Holotype: HUJ 11398. Paratypes: BMNH 1985.3.19.1 (1), HUJ 10604 (1). Distribution: Red Sea.

\section{Atrobucca kyushini Sasaki and Kailola, 1988}

Atrobucca kyushini Sasaki and Kailola, 1988: 263, figs. 1, 2A, 3 (Off Kuching, Borneo, Malaysia). Holotype: HUMZ 107200. Paratypes: BMNH 1987.6.4.1 (1), 1987.6.4.2 (1); HUMZ 33633 (1), 46810 (1), 46945 (1), 50132 (1), 107201-02 (1, 1), 107205-06 (1, 1); NSMT-P 50538-39 $(1,1)$.

Distribution: Off northwestern coast of Borneo, South China Sea.

\section{Atrobucca marleyi (Norman, 1922)}

Sciaena marleyi Norman, 1922: 319 (Off St. John's R., Natal, South Africa). Holotype (unique): BMNH 1922.1.13.37.

Distribution: Mozambique south to Natal.

\section{Atrobucca nibe (Jordan and Thompson, 1911)}

Pseudotolithus brunneolus Jordan and Richardson, 1909: 191, Pl. 71 (Kaohsiung, Taiwan). Holotype: FMNH 52174 [ex CM 327]. Paratypes: FMNH 59521 (1), CAS-SU 21185 (1).

Sciaena nibe Jordan and Thompson, 1911: 258, fig. 4 (Wakanoura, Japan). Holotype: USNM 67331. Paratypes: CAS-SU 25010 (2).

Nibea pingi Wang, 1935: 448, fig. 32 (Chefoo, Shantung Prov., China). Holotype (unique): Mus. Biol. Lab. Sci. Soc. China, Nanking 10320.

Distribution: Indo-West Pacific: Mozambique and Natal, South Africa, India; China, Japan, Philippines, Indonesia, and off northern Australia.

Comments: Pseudotolithus brunneolus Jordan and Richardson, 1909 has been suppressed to maintain Sciaena nibe (Opinion 1272; Bull. Zool. Nom. 1984, 41 p. 26-27).

Atrobucca trewavasae Talwar and Sathiarajan, 1975 
Atrobucca trewavasae Talwar and Sathiarajan, 1975: 575, fig. 1 (Bay of Bengal off Madras, India, depth 250 meters). Holotype: ZSI F7131/2. Paratypes: (9) BMNH 1974.7.5.4-5; ZSI F7133/2-7137/2 and 7139-7140/2 (7).

Distribution: Bay of Bengal.

\section{Genus Austronibea Trewavas, 1977}

Austronibea Trewavas, 1977: 361, 373. Feminine. Type species Austronibea oedogenys Trewavas, 1977. Type by original designation.

Northern Australia and New Guinea in shallow coastal waters and estuaries; monotypic.

\section{Austronibea oedogenys Trewavas, 1977}

Austronibea oedogenys Trewavas, 1977: 374, Pl. 4 (fig. 25) (Repulse Bay, Queensland, Australia). Holotype: QM I.12691. Paratypes: BMNH 1977.4.5.1 ex CSIRO A 4076 (1); CSIRO A 4076 (11), C 3415 (1), C 4532 (1); RMNH 27284 (1).

Distribution: Northern and northwestern Australia and southern New Guinea.

\section{Genus Bahaba Herre, 1935}

Bahaba Herre, 1935: 603. Feminine. Subgenus of Otolithes, Type species Otolithes (Bahaba) lini Herre, 1935. Type by original designation.

West Pacific. Three species.

\section{Bahaba chaptis (Hamilton, 1822)}

Bola chaptis Hamilton, 1822: 77, 368, Pl. 10 (fig. 25) (estuaries of the Ganges). Neotype: ZSI F6229/2. Neotype designated by Talwar and Datta (1972: 171).

Bola chaptis var. nuria Hamilton, 1822: 78, 368, Jassore, India. No types known.

Distribution: Western Bengal and Burma in coastal waters and lower parts of rivers.

\section{Bahaba polykladiskos (Bleeker, 1852)}

Corvina polykladiskos Bleeker, 1852: 420 (Bandjarmasin, Borneo, Indonesia). Holotype (unique): RMNH 5983.

Distribution: Mekong basin and Borneo.

\section{Bahaba taipingensis (Herre, 1932)}

Nibea taipingensis Herre, 1932: 436 (Market at Taiping, Kwangtung, China). Holotype: CASSU 25723. Paratypes: CAS-SU 69834 ex CAS-SU 25723 (2).

Nibea flavolabiata Lin, 1935: 14, fig. 7 (Chusan, China, East China Sea). Holotype (unique): Chekiang Fisheries Exp. Station, mounted specimen.

Otolitheslini Herre, 1935: 603 (Canton market, China [caught below delta region, not far from Hong Kong]). Holotype: CAS-SU 13984. Paratypes: FMNH 47086 ex CM (1), CAS-SU $16703(1)$.

Distribution: South China only. From the Yangtze River southwards to Hong Kong.

\section{Genus Bairdiella Gill, 1861}

Bairdiella Gill, 1861a: 33. Feminine. Type species Bodianus argyroleucus Mitchill, 1815. Type by monotypy.

Amphi-American inshore waters, estuaries, and coral reefs over sandy to muddy bottoms.

Eight species according to Chao (1978) with the inclusion of Corvula. Marceniuk et al. (2019) revised the western Atlantic species. Seven species.

\section{Bairdiella armata Gill, 1863}

Bairdiella armata Gill, 1863b: 164 (Western coast of Central America). Holotype (unique): USNM 35134. 
Corvina (Homoprion) acutirostris Steindachner, 1875b: 56, Pl. 4 (Panama, Atlantic coast). Syntypes: NMW 72848 (1), 78573 (2).

Corvina fulgens Vaillant and Bocourt, 1883: 164, Pl. 6 (fig. 2) (La Union, El Salvador eastern Pacific). Syntypes: MNHN A-0975 (2).

Distribution: Eastern Pacific from Gulf of California to Panama.

\section{Bairdiella chrysoura (Lacepède, 1801)}

Perca punctatus Linnaeus (ex Garden), 1766: 482 (South Carolina, U.S.A.). Syntypes: LS 108-109 (left half-skins). Objectively invalid, nomen preoccupatum, preoccupied by Perca punctata Linnaeus 1758 [= Cephalopholis fulva (Linnaeus, 1758)].

Dipterodon chrysourus Lacepède, 1801: 166, 174 (Carolina, U.S.A.). No types known.

Bodianus argyroleucus Mitchill, 1815: 417, Pl. 6 (fig. 9) (New York, U.S.A.). No types known.

Bodianus exiguus Mitchill, 1815: 419 (New York, U.S.A.). No types known.

Bodianus pallidus Mitchill, 1815: 420 (New York, U.S.A.). No types known.

Homoprion xanthurus Holbrook, 1855: 170, pl. 24 (not Leiostomus xanthurus Lacepède, 1802).

Homoprion subtruncatus Gill, 1861a: 33 (South Carolina, U.S.A.). Based on Leiostomus xanthurus of Storer (1846: 69).

Distribution: New York to southern Florida and northern Gulf of Mexico.

\section{Bairdiella ensifera (Jordan and Gilbert, 1882)}

Sciaena ensifera Jordan and Gilbert, 1882a: 313 (Pacific Panama; Punta Arenas, Costa Rica). Syntypes: USNM 29316 (1), 29442 (1), 29464 (1), 29506 (1), 29526 (1).

Distribution: Eastern Pacific from Mexico to Peru.

\section{Bairdiella goeldi Marceniuk, Molina, Caires, Rotundo, Wosiacki and Oliveira, 2019}

Bairdiella goeldi Marceniuk, Molina, Caires, Rotundo, Wosiacki and Oliveira, 2019: 6, fig. 4a (Furo da Ostra, Bragança, Pará, Brazil). Holotype: MPEG 33641. Paratypes: MPEG 32860 (1); MPEG 33653 (1); MPEG 33654 (4); MPEG 34510(2); MPEG 33627 (2); MPEG 33628 (1); MPEG 33615(1); AZUSC 4926(9); LBP 19376(4).

Distribution: Widely distributed on the Atlantic coast of Brazil, from at least the equatorial northern state of Pará to Santa Catarina. The southern limit of occurrence is apparently determined by the absence of mangrove forests in estuaries.

\section{Bairdiella icistia (Jordan and Gilbert, 1882)}

Sciaena icistia Jordan and Gilbert, 1882d: 356 (Mazatlán, Sinaloa, w. Mexico). Syntypes: USNM 28182 (1), 28228 (1), 28275 (1, lost), 28368 (1), 29566 (1), 29613 (1, lost), 29615 (1), 29775 (1), 29790 (1).

Distribution: Gulf of California to Chiapas, Mexico.

\section{Bairdiella ronchus (Cuvier, 1830)}

Corvina ronchus Cuvier in Cuvier and Valenciennes, 1830: 107 (Lake Maracaibo, Venezuela; Dominican Republic; Cuba; Suriname). Lectotype: MNHN 7634. Paralectotypes: MNHN 0095 (1) Dominican Republic, 5345 (2) Suriname, 7637 (1), A-5543 (1) Martinique. Lectotype selected by Marceniuk et al. (2019: 13).

Corvina subaequalis Poey, 1875: 58 (Cuba). Holotype (unique): ?ZMB lost.

Sciaena (Bairdiella) bedoti Regan, 1905: 391, Pl. 6 (fig. 1) (Cuba). Syntypes: BMNH 1905.3.18.2 ex MHNG (1), MHNG 678.01 (1).

Distribution: Greater Antilles and Central America to Brazil.

\section{Bairdiella veraecrucis Jordan and Dickerson, 1908}

Bairdiella veraecrucis Jordan and Dickerson, 1908: 16, fig. 1 (Veracruz, Mexico). Holotype: USNM 61676. Paratypes: CAS-SU 20654 (2). 
Distribution: Northern Greater Caribbean Province, Gulf of Mexico.

Genus Boesemania Trewavas, 1977

Boesemania Trewavas, 1977: 309. Feminine. Type species Johnius microlepis Bleeker, 1858. Type by original designation.

West Pacific, in flowing waters of large rivers and in the deep-water pools of the Mekong River and rivers of Indonesia. Monotypic.

\section{Boesemania microlepis (Bleeker, 1858)}

Johnius microlepis Bleeker, 1858: 11 (Palembang, Mussi River mouth, Sumatra, Indonesia). Holotype (unique): RMNH 6043 (125 mm TL spec. of 2, poor condition).

Otolithoides aeneocorpus Fowler, 1935: 150, fig. 119 (Bangkok, Thailand). Holotype: ANSP 62510. Paratypes: ANSP 62511-24 (14), BPBM 10591 ex ANSP 62525 (1).

Distribution: From Thailand to Vietnam and Indonesia.

\section{Genus Callaus Jordan, 1889}

Callaus Jordan in Jordan and Eigenmann, 1889: 395, 401. Feminine. Subgenus of Sciaena, type species Corvina deliciosa Tschudi, 1846. Type by original designation.

Eastern Pacific.

Comments: The genus is here regarded as valid following Aguilera et al. (2016).

\section{Callaus deliciosa (Tschudi, 1846)}

Corvina deliciosa Tschudi, 1846: 9 (Market at Lima, Peru). Syntypes: (several) ZMB 894 (1).

?Umbrina imberbis Günther, 1873: 101 (Iquique, Chile). Holotype (unique): whereabouts unknown.

Sciaena suavis de Buen, 1961: 17 (Arica, Chile). Holotype: EBMC 10377 (lost). Paratypes: EBMC 10378 (1) (lost).

Distribution: Peru and Chile.

Comments: Synonymy from Kong and Valdés (1990).

\section{Genus Cheilotrema Tschudi, 1846}

Cheilotrema Tschudi, 1846: 13. Neuter. Type species Cheilotrema fasciatum Tschudi, 1846. Type by monotypy.

Rhinoscion Gill, 1861b: 78. Masculine. Type species Amblodon saturnus Girard, 1858. Type by monotypy.

Eastern Pacific, near sandy bottoms, often in caves and crevices of exposed coasts and open bays; two species.

\section{Cheilotrema fasciatum Tschudi, 1846}

Cheilotremafasciatum Tschudi, 1846: 13, Pl. 1 (Between Callao and Huacho, Peru). Holotype (unique): ZMB 1022 or ZMB 1023.

Distribution: Along the coast of Peru and Chile.

\section{Cheilotrema saturnum (Girard, 1858)}

Amblodon saturnus Girard, 1858a: 98 (San Diego, California, U.S.A.). Syntypes: USNM 638 (orig. 3, now 2).

Corvina (Johnius) jacobi Steindachner, 1879: 121 (San Diego, California). Syntypes: (several) NMW.

Distribution: Point Conception to central Baja California and upper Gulf of California.

Comments: Synonyms as reported by Jordan et al. (1930). 


\section{Genus Chrysochir Trewavas and Yazdani, 1966}

Chrysochir Trewavas and Yazdani, 1966: 250. Feminine. Type species Otolithus aureus Richardson, 1846. Type by original designation

Indo-West Pacific in shallow coastal waters; monotypic.

\section{Chrysochir aureus (Richardson, 1846)}

Otolithus aureus Richardson, 1846: 224 (Canton, China). Lectotype: BMNH 1975.11.17.1 (half skin). Paralectotypes: USNM (1, apparently lost). Lectotype selected by Trewavas 1977: 345 .

Sciaena ophiceps Alcock, 1889: 300 (Off Mahanadi delta, depth 5-9 fathoms; off Godavari delta, depth 4-5 fathoms; Orissa coast). Syntypes: BMNH 1890.12.4.6-7 ex ZSI (2); ZSI F12099 to 12101 (3), 12103 (1), 12170-71 (1, 1), 12336 (1), 12381 (1).

Sciaena incerta Vinciguerra, 1926: 578 (Sarawak state, Borneo, East Malaysia). Syntypes: (2) MSNG 23308 (1).

Johnius birtwistlei Fowler, 1931: 446, fig. (Clyde Terrace market, Singapore). Holotype: ANSP 53467 (not separated, in jar with paratypes). Paratypes: ANSP 53468-69 (2).

Distribution: Bay of Bengal to China Sea.

\section{Genus Cilus Delfin, 1900}

Cilus Delfin, 1900: 55. Masculine. Type species Cilus montti Delfin, 1900. Type by monotypy.

Eastern Pacific; monotypic.

\section{Cilus gilberti (Abbott, 1899)}

Sciaena gilberti Abbott, 1899: 355 (Callao, Peru). Holotype: CAS-SU 11978 not 11918 as published. Paratypes: CAS-SU 6199 (1).

Cilus montti Delfin, 1900: 56, fig. 1 (Talcahuano, San Vicente, Concepción, Chile). No types known.

Distribution: Peru to Chile and Galápagos Islands.

Comments: Previously placed in Haemulidae (Fowler, MS), family position has been established by Oyarzún et al. (1985).

\section{Genus Collichthys Günther, 1860}

Collichthys Günther, 1860: 312. Masculine. Type species Sciaena lucida Richardson, 1844. Type by subsequent designation. Type designated by Jordan and Eigenmann, 1889: 348.

Hemisciaena Bleeker, 1863a: 140. Feminine. Type species Sciaena lucida Richardson, 1844. Type by monotypy.

West Pacific in sublittoral zone and in estuaries over sandy-muddy bottoms; two species.

\section{Collichthys lucidus (Richardson, 1844)}

Sciaena lucida Richardson, 1844: no p., Pl. 44 (figs. 3-4) (China Seas). Syntypes: BMNH 1848.3.18.107-108 (1 of 2).

Sciaena meygun Basilewsky, 1855: 222 (Beijing, China). No types at ZIN.

Collichthys chinensis Steindachner, 1866: 475. Appears as "Collichthys lucidus Richds. An nov. spec.? (C. chinensis)."

Collichthys fragilis Jordan and Seale, 1905a: 522, fig. 4 (Shanghai, China). Holotype (unique): USNM 52080.

Distribution: South China Sea to Lüshunkou (=Port Arthur), Liaodong Peninsula.

\section{Collichthys niveatus Jordan and Starks, 1906}

Collichthys niveatus Jordan and Starks, 1906: 519, fig. 2 (Port Arthur, Manchuria, China).

Holotype: USNM 55632. Paratypes: CAS-SU 9997 not 9884 as published (16).

Distribution: Yellow Sea to East China Sea. 


\section{Genus Corvula Jordan and Eigenmann, 1889}

Corvula Jordan and Eigenmann, 1889: 377. Feminine. Type species Johnius batabanus Poey, 1860. Type by original designation.

Vacuoqua Jordan and Evermann, 1927: 506. Feminine. Type species Corvina macrops Steindachner, 1876. Type by original designation.

A junior synonym of Bairdiella according to Chao (1978). Valid according to Allen and Robertson (1994). Amphi-American in rocky coastal areas. Three species.

Corvula batabana (Poey, 1860)

Johnius batabanus Poey, 1860: 184 (Batabano, south coast of Cuba). Syntypes: MCZ 10926$27(1,1), 21957(1)$.

Corvula sialis Jordan and Eigenmann, 1889: 379 (Key West, Florida, U.S.A.). Holotype (unique): USNM 26575.

Distribution: South Florida, Bay of Campeche, Greater Antilles and Virgin Islands.

\section{Corvula macrops (Steindachner, 1875)}

Corvina macrops Steindachner, 1875b: 52, Pl. 2 (Panama [Pacific]). Syntypes: NMW 78572 (1).

Distribution: Eastern Pacific from Gulf of California to Ecuador and Galapagos Islands.

\section{Corvula sanctaeluciae Jordan, 1890}

Corvula sanctaeluciae Jordan, 1890: 649 (St. Lucia, West Indies). Holotype (unique): USNM 41732.

Distribution: Eastern Florida, Cuba, Bay of Campeche to Guianas.

\section{Genus Ctenosciaena Fowler and Bean, 1923}

Ctenosciaena Fowler and Bean, 1923: 15. Feminine.Subgenus of Sciaena, type species Sciaena (Ctenosciaena) dubia Fowler and Bean, 1923. Type by original designation.

Amphi-American in offshore waters over hard sandy bottom; two species.

\section{Ctenosciaena gracilicirrhus (Metzelaar, 1919)}

Umbrina gracilicirrhus Metzelaar, 1919: 72, fig. 24 (Coast of Venezuela). Holotype (unique): ZMA 113103.

Sciaena dubia Fowler and Bean, 1923: 16 (No locality). Holotype (unique): USNM 83309.

Distribution: Nicaragua along the Caribbean coast and Atlantic coasts of South America to southern Brazil.

\section{Ctenosciaena peruviana Chirichigno, 1969}

Ctenosciaena peruviana Chirichigno, 1969: 1, fig. (Coast of Peru, $5^{\circ} 41^{\prime} \mathrm{S}, 81^{\circ} 14^{\prime} \mathrm{W}$, depth 187 meters). Holotype: IMARPE 1248. Paratypes: IMARPE 1193-98 (1, 2, 5, 2, 1, 1).

Distribution: Off Ecuador and Peru.

\section{Genus Cynoscion Gill, 1861}

Cynoscion Gill, 1861c: 81. Masculine. Type species Johnius regalis Bloch and Schneider, 1801. Type by original designation.

Cestreus Gronow in Gray, 1854: 49. Masculine. Type species Cestreus carolinensis Gronow, 1854. Type by monotypy. Objectively invalid; preoccupied by Cestreus McClelland, 1842 in fishes; apparently not replaced.

Apseudobranchus Gill, 1862: 18. Masculine. Type species Otolithus toeroe Cuvier, 1830. Type by original designation.

Archoscion Gill, 1862: 18. Masculine. Type species Otolithus analis Jenyns, 1842. Type by original designation. 
Buccone Jordan and Evermann, 1896: 394 Neuter. Subgenus of Cynoscion, type species Cestreus praedatorius Jordan and Gilbert, 1889. Type by original designation.

Symphysoglyphus Miranda-Ribeiro, 1913: 43. Masculine. Type species Ancylodon bairdii Steindachner, 1879.

Paralarimus Fowler and Bean, 1923: 18. Masculine. Type species Paralarimus patagonicus Fowler and Bean, 1923. Type by original designation.

Eriscion Jordan and Evermann, 1927: 506. Masculine. Subgenus of Cynoscion, type species Otolithus nebulosus Cuvier, 1830. Type by original designation.

Eastern Pacific (12 species) and western Atlantic (13 species) in estuarine and inshore waters, with 25 species.

\section{Cynoscion acoupa (Lacepède, 1801)}

Cheilodipterus acoupa Lacepède, 1801: 540, 546 (Cayenne, French Guinea). Holotype (unique): MNHN 0000-5502.

Johnius thorax Bloch and Schneider, 1801: 77 (Suriname). Holotype (unique): MNHN 00005502.

Lutjanus cayanensis Lacepède, 1802: 196, 240 (Cayenne, French Guiana). Syntypes: MNHN 0000-5502 (1, holotype of Bauchot), A-4562 (2), A-5617 (2).

Otolithus rhomboidalis Cuvier, 1829: 173 (Cayenne, French Guiana). No types known.

Otolithus toeroe Cuvier in Cuvier and Valenciennes, 1830: 72, Pls. 103, 138 (Cayenne, French Guiana; Suriname; Brazil; Lake Maracaibo).Syntypes: MNHN 0000-4616 (1) Cayenne, 0000-5500 (1) Brazil, 0000-5502 (1) Cayenne, A-0456 (1, dry) Brazil, A-0528 (1, dry) Brazil, A-5617 (1, dry) Lake Maricaibo; SMF 2167 ex MNHN in 1830 (1).

Otolithus cayennensis Vaillant and Bocourt, 1883: 156 (La Union, El Salvador) Holotype (unique): whereabouts unknown. New name or emendation of Lutjanus cayanensis Lacepède. Paralarimus patagonicus Fowler and Bean, 1923: 18 (Northern Patagonia). Holotype (unique): USNM 83222.

Cynoscion maracaiboensis Schultz, 1949: 160, fig. 20 (Río Agua Caliente, 2-3 kilometers above Lake Maracaibo, Venezuela). Holotype: USNM 121742. Paratypes: MCZ 37219 [ex USNM 121744] (1); UMMZ 157676 (1); USNM 121743 (1), 121744 (orig. 2, now 1), 121745 (1).

Distribution: Tropical western Atlantic to Argentina.

Comments: Under the account of Paralarimus patagonicus Chao (2002a: 8) wrote: "The specimen was collected during the Wilkes expedition and catalogued on 1 March 1919. I suspect the stated locality of the holotype was incorrectly registered." Johnius thorax Bloch and Schneider, 1801 was published the same year as Cheilodipterus acoupa Lacepède, 1801, but its actual date of publication is unknown and therefore placed at 31 December 1801. Lacepède published his work the 18 October 1801.

\section{Cynoscion albus (Günther, 1864)}

Otolithus albus Günther, 1864: 149 (Chiapam, Guatemala). Holotype (unique): BMNH 1864.1.26.240.

Distribution: Southern Mexico to Peru.

\section{Cynoscion analis (Jenyns, 1842)}

Otolithus analis Jenyns, 1842: 164 (Callao, Peru). Holotype (unique): BMNH 1917.7.14.44.

Otolithus peruanus Tschudi, 1846: 10 (coast of Peru). Holotype: ZMB 864.

Distribution: Ecuador, Peru and Chile. Inhabits coastal waters. Juveniles penetrate estuaries and shallow bays. Feeds principally on shrimps and fishes. Also caught with encircling nets. Comments: Taxonomic decision of Hildebrand (1946). 


\section{Cynoscion arenarius Ginsburg, 1930}

Cynoscion arenarius Ginsburg, 1930: 83, fig. 7 (Off entrance to harbor, Galveston, Texas, U.S.A.). Holotype (unique): USNM 89395.

Distribution: Florida and Gulf of Mexico to Bay of Campeche.

\section{Cynoscion guatucupa (Cuvier, 1830)}

Otolithus guatucupa Cuvier in Cuvier and Valenciennes, 1830: 75, Pl. 104 (Brazil and Montevideo, Uruguay). Syntypes: MNHN 0000-7517 (2) Montevideo, MNHN “holotype” (1, lost) Brazil.

Distribution: Surinam to Brazil.

Comments: This species is known by several authors as Cynoscion striatus (Chao, 1987; Figuereido, 1992).

\section{Cynoscion jamaicensis (Vaillant and Bocourt, 1883)}

Otolithus jamaicensis Vaillant and Bocourt, 1883: 156, Pl. 6 (figs. 1a-d) (Jamaica). Holotype (unique): MNHN A-0557.

Archoscion petranus Miranda Ribeiro, 1913: 42 (Campo Grande, Brazil). No types known.

Distribution: Antilles to Brazil.

\section{Cynoscion leiarchus (Cuvier, 1830)}

Otolithus leiarchus Cuvier in Cuvier and Valenciennes, 1830: 78 (Brazil; French Guiana). Syntypes: MNHN 0000-5503 (2) Brazil, A-2690 (1, dry) Brazil, A-5422 (1, dry) Brazil; ?NMW 31096 (1).

Distribution: Central western Atlantic. Nicaragua and Panama to Santos, Brazil.

\section{Cynoscion microlepidotus (Cuvier, 1830)}

Otolithus microlepidotus Cuvier in Cuvier and Valenciennes, 1830: 79 (Suriname). Holotype (unique): ZMB.

Ancylodon bairdii Steindachner, 1879b: 33 (Santos, Brazil). Syntypes: (several) NMW 51130 (1). Species first appeared as above as Ancylodon (?) bairdii, later as Otolithus (?) bairdi when illustrated and described in more detail in Steindachner 1879: 40, Pl. 1 (fig. 2).

Distribution: Southwestern Atlantic: Venezuela south to Santos, Brazil.

\section{Cynoscion nannus Castro-Aguirre and Arvizu-Martinez, 1976}

Cynoscion nannus Castro-Aguirre and Arvizu-Martinez, 1976: 323, fig. 1 (18 mile southwest

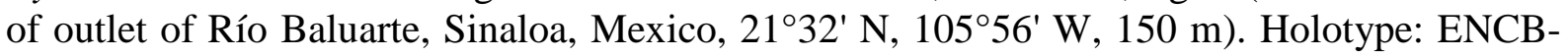
IPN 4109 ex 6982. Paratypes: ENCB-IPN 4108 ex 6082 (9).

Distribution: Central eastern Pacific: offshore Mexico between 100-812 m.

\section{Cynoscion nebulosus (Cuvier, 1830)}

Labrus squeteague var. maculatus Mitchill, 1815: 396 (New York, U.S.A.). No types known. Objectively invalid, nomen praeoccupatum, preoccupied by Labrus maculatus Bloch, 1792 (=Labrus bergylta Ascanius, 1767).

Otolithus nebulosus Cuvier in Cuvier and Valenciennes, 1830: 79 (no locality stated). Holotype (unique): MNHN 0000-7527.

Otolithus carolinensis Cuvier in Cuvier and Valenciennes, 1830: 475 (Charleston, South Carolina, U.S.A.). Holotype (unique): MNHN 0000-7507 (poor condition).

Otolithus drummondii Richardson, 1836: 70, footnote (New Orleans, Louisiana, U.S.A.). Holotype (unique): whereabouts unknown.

Distribution: New York to entire Gulf of Mexico.

\section{Cynoscion nortoni Béarez, 2001}


Cynoscion nortoni Béarez, 2001: 60, figs. 1-3 (Puerto López, Manabí, Ecuador, 1³3' S, 8049' W, depth 100-200 meters, fish market). Holotype: MNHN 1999-0961. Paratypes: CAS 208889 (1), MCZ 156126 (1), MNHN 1999-0962 (7), USNM 357280 (1).

Distribution: Coasts of Ecuador and Peru.

\section{Cynoscion nothus (Holbrook, 1848)}

Otolithus nothus Holbrook, 1848: 46, Pl. 8 (South Carolina, U.S.A.). No types known. Also appeared in Holbrook 1855: 134, Pl. 19 (fig. 1) and in the second edition, 1860: 134, Pl. 19 (fig. 1).

Distribution: Maryland to entire Gulf of Mexico.

\section{Cynoscion othonopterus Jordan and Gilbert, 1882}

Cynoscion othonopterum Jordan and Gilbert, 1882c: 274 (Punta San Felipe, Gulf of California, Mexico). Holotype (unique): USNM 29385.

Cynoscion squamipinnis Streets, 1877: 48 (Gulf of California).

Distribution: Gulf of California to Panama.

\section{Cynoscion parvipinnis Ayres, 1861}

Cynoscion parvipinnis Ayres, 1861: 156, fig. 48 (Coast of Baja California, $27^{\circ} \mathrm{N}$, Mexico). Holotype (unique): No types known.

Otolithus magdalenae Steindachner, 1875b: 62 (Magdalena Bay, Baja California, Mexico). Syntypes: (several) MCZ 10880 (4), 10897 (2); NMW 31152 (1), 32256 (1), 32268 (1); USNM 153617 ex MCZ 10880 (1); ZMUC 26 (1).

Distribution: Gulf of California to Mazatlán, Mexico.

Comments: Otolithus magdalenae is a synonym of Cynoscion parvipinnis following Chao (1995). Typographical error in Chao (2002), meant to be a synonym. Not in alphabetical order and the species does not appear in the key.

\section{Cynoscion phoxocephalus Jordan and Gilbert, 1882}

Cynoscion phoxocephalum Jordan and Gilbert, 1882a: 318 (Pacific Panama). Syntypes: USNM 29296 (1), 29339 (1), 29389 (1), 29724 (1).

Distribution: El Salvador to Peru.

\section{Cynoscion praedatorius (Jordan and Gilbert, 1889)}

Cestreus praedatorius Jordan and Gilbert in Jordan and Eigenmann, 1889: 363 Panama. Syntypes: MCZ 10901-02 (3).

Distribution: From Costa Rica and Panama. Inhabits coastal waters.

\section{Cynoscion regalis (Bloch and Schneider, 1801)}

Johnius regalis Bloch and Schneider, 1801: 75(New York, U.S.A.). Holotype (unique): ZMB 8700 (stuffed).

Roccus comes Mitchill, 1814: 25 (New York, U.S.A.). No types known.

Labrus squeteague Mitchill, 1815: 396, Pl. 2 (fig. 6) (New York, U.S.A.). No types known.

Otolithus thallapinus Holbrook, 1848: Pl. 5 (fig. 2) (South Carolina). No types known.

Cestreus carolinensis Gronow in Gray, 1854: 49 (Off Carolina, U.S.A., western North Atlantic). Holotype: BMNH 1853.11.12.42 Gronovius coll. (skin).

Otolithus thalassinus Holbrook, 1855: 1: 132, Pl. 18 (fig. 2) (Charleston, South Carolina, U.S.A.). No types known.

Otolithus obliquatus Sauvage (ex Valenciennes), 1879: 209 (Martinique Island, West Indies). Lectotype: MNHN 0000-7632. Paralectotypes: MNHN 1987-0151 ex MNHN 0000-7632 (1). Lectotype selected by Randall and Cervigón (1968: 1: 182).

Distribution: Nova Scotia to northern Florida. 


\section{Cynoscion reticulatus (Günther, 1864)}

Otolithus reticulatus Günther, 1864: 1: 149 (San José and Chiapam, Guatemala). Holotype (unique): BMNH 1864.1.26.324.

Distribution: Gulf of California to Panama. Inhabits coastal waters and estuaries with high salinities.

\section{Cynoscion similis Randall and Cervigón, 1968}

Cynoscion similis Randall and Cervigón, 1968: 1: 179, fig. 2 (Porlamar fish market, Isla de Margarita, Nueva Esparta, Venezuela). Holotype: USNM 201382. Paratypes: UF ex UMML 4299 (1), 212502 ex UMML 12502 (1); UPR 2868 (1); MOBR-EDIMAR-P-1978 (4).

Distribution: Isla de Margarita (Venezuela).

\section{Cynoscion squamipinnis (Günther, 1867)}

Otolithus squamipinnis Günther, 1867a: 601 (Pacific Panama). Syntypes: BMNH 1865.7.20.20-21 (2). Described in more detail in Günther 1868: 429.

Distribution: Gulf of California to northern Peru.

\section{Cynoscion steindachneri (Jordan, 1889)}

Cestraeus steindachneri Jordan in Jordan and Eigenmann, 1889: 372 (Ponta Curuçá, Pará, Brazil). Holotype (unique): MCZ 10922.

Distribution: Central America and Brazil.

\section{Cynoscion stolzmanni (Steindachner, 1879)}

Otolithus stolzmanni Steindachner, 1879b: 33(Tumbes, Peru). Syntypes: (several) NMW. Species later illustrated and described in more detail in Steindachner 1879c: 35, Pl. 2 (fig. 1).

Distribution: Mexico to Peru.

\section{Cynoscion striatus (Cuvier, 1829)}

Otolithus striatus Cuvier, 1829: 1: 173 (Brazil). No type known.

Perca argentea Larrañaga, 1923: 384 (Uruguay). Also appeared as P. argentea vel 10-vittata in Devincenzi, 1925: 305, 321. Objectively invalid, nomen preoccupatum, preoccupied by Perca argentea Linnaeus, 1758 (=Terapon theraps Cuvier, 1829) but nomen oblitum and preoccupied by Perca argentea Bennet, 1830 [=Kuhlia mugil (Forster, 1801)].

Perca vigintiuniradiata Larrañaga, 1923: 384 (Uruguay). No types known.

Distribution: Southern Brazil to central Argentina.

\section{Cynoscion virescens (Cuvier, 1830)}

Otolithus virescens Cuvier in Cuvier and Valenciennes, 1830: 72 (Suriname). Holotype (unique): ZMB.

Otolithus microps Steindachner, 1879b: 33 (Porto Alegre, Brazil). Holotype (unique): NMW 31111. Species later illustrated and described in more detail in Steindachner 1879c: 38, Pl. 8. Distribution: Southwestern Atlantic.

\section{Cynoscion xanthulus Jordan and Gilbert, 1882}

Cynoscion xanthulum Jordan and Gilbert, 1882e: 460 (Mazatlán, Sinaloa, w. Mexico). Holotype (unique): USNM 28109.

Distribution: Gulf of California to Acapulco, Mexico; also found in the Salton Sea, South California, a shallow and highly saline lake.

\section{Genus Daysciaena Talwar, 1971}

Daysciaena Talwar, 1971a: 1: 192. Feminine. Type species Corvina albida Cuvier, 1830. Type by original designation.

Northern Indian Ocean, in coastal waters and estuaries; monotypic. 


\section{Daysciaena albida (Cuvier, 1830)}

Corvina albida Cuvier in Cuvier and Valenciennes, 1830: 93 (Puducherry and Mahé, India). Syntypes: MNHN 0000-7523 (1) Mahé, 0000-7524 (1) Puducherry, A-8825 (1, poor condition) Mahé, 0000-9805 (1, skin) Puducherry.

Corvina neilli Day, 1865: 55 (Cochin, Malabar, India). No types known.

Dendrophysa hooghliensis Sinha and Rao, 1969: 77, figs. 1-4 (Hooghly River Estuary, West Bengal, India). Holotype: ZSI F5843/2. Paratypes: BMNH 1967.7.31.12-15 (4); USNM 203256 (2); ZSI F5844/2 (2).

Distribution: India, Sri Lanka (marine and freshwaters).

Comments: Placed in the genus Nibea by de Bruin et al. (1995).

\section{Genus Dendrophysa Trewavas, 1964}

Dendrophysa Trewavas, 1964: 1: 110. Feminine. Type species Umbrina russellii Cuvier, 1829. Type by original designation.

Indo-West Pacific on shallow coastal mud banks. Monotypic.

\section{Dendrophysa russelii (Cuvier, 1829)}

Umbrina russelii Cuvier, 1829: 174 (Vishakhapatnam [Vizagapatam], India). Neotype: BMNH 1889.2.1.3087 designated by Trewavas 1964: 111. Name usually spelled russellii or russelli, but the correct original spelling is russelii.

Umbrina kuhlii Cuvier in Cuvier and Valenciennes, 1830: 179 (Java, Indonesia). Holotype (unique): RMNH 682.

Distribution: Pakistan, India, Sri Lanka, and Andaman Islands to China Sea south to Australia.

\section{Genus Elattarchus Jordan and Evermann, 1896}

Elattarchus Jordan and Evermann, 1896: 397. Masculine. Type species Odontoscion archidium Jordan and Gilbert, 1882. Type by original designation.

Eastern Pacific in shallow coastal waters. Uncommon species. Inhabits shallow waters, often along sandy bays and in lagoons; rarely found in estuaries. Monotypic.

\section{Elattarchus archidium (Jordan and Gilbert, 1882)}

Odontoscion archidium Jordan and Gilbert, 1882a: 317 (Panama Bay, Pacific Panama). Syntypes: USNM 29266 (1), 29480 (1, lost), 29518 (1).

Odontoscion australis Hildebrand, 1946: 280, fig. 59 (Lobos de Tierra Bay, Peru). Holotype: USNM 128023. Paratypes: USNM 128024 (3), Gov't of Peru ex USNM 128024.

Distribution: Gulf of California to Peru.

\section{Genus Eques Bloch, 1793}

Eques Bloch, 1793: 90. Masculine. Type species Eques americanus Bloch, 1793. Type by monotypy.

Equetus Rafinesque, 1815: 86. Masculine. Type species Eques americanus Bloch, 1793. Type by being a replacement name.

Endemic to the tropical western Atlantic mainly on coral reefs. Monotypic according to Sasaki (1989); two species according to Chao (1978) and Acero et al. (1984); four species according to Robins et al. (1986).

Comments: The Eschmeyer's Catalog of Fishes reports Eques Bloch, 1793 preoccupied by Eques Linnaeus 1758 in Lepidoptera. However, Balletto et al. (2014: 79) wrote: "unfortunately enough, when ICZN (1957) issued its Op. 450, thereby changing Op. 124 and 279, it validated at subgenus rank all Linné's subdivisions of Phalaena (itself suppressed under the Plenary Powers), i.e. Alucita, Bombyx, Geometra, Noctua, Pyralis, Tinea and Tortrix, but failed to do the same for many other similarly published names. More in 
particular, all butterflies described by Linnaeus in 1758 (Syst. Nat., ed x), still fall under the provisions of Op. 124, 279 and are considered to have been published under the Genus Papilio, so that none of its Linnean divisions (Barbarus, Eques, Heliconius, Danaus, Nymphalis, Plebejus) are deemed nomenclaturally valid under that author and date. In other words, such names are to be treated as "interpolated names" devoid of any nomenclatural status." Therefore, Eques Bloch, 1793 is a valid genus of fishes. Reversal of precedence (art. 23.9.1) cannot be applied because Eques was used as valid name after 1899 in several instances (e.g. Miranda Ribeiro 1915; Fowler 1919; Beebe and Tee-Van 1928). The combination Eques lanceolatus is also used in the Merriam-Webster dictionary. In order to continue the usage of Equetus Rafinesque the matter must be referred to the ICZN Commission to rule under the plenary power (art. 23.9.3 and art. 81).

\section{Eques lanceolatus (Linnaeus, 1758)}

Chaetodon lanceolatus Linnaeus, 1758: 277 (India). No types known.

Eques americanus Bloch, 1793: 91, Pl. 347 (fig. 1) (Western Atlantic). Holotype (unique): ZMB 905.

Eques balteatus Cuvier, 1829: 175 (America). No types known. Cuvier specimens: MNHN 0000-7449 (1) Martinique.

Sciaena edwardi Gronow in Gray, 1854: 53 (Indian Sea). No types known.

Distribution: North Carolina, Bermuda, northern Gulf of Mexico to Brazil.

Comments: The type locality Chaetodon lanceolatus Linnaeus, 1758 is the West Indies, as description is based on the Ribband-Fish of Edwards (1751) described and illustrated from the Caribbean Islands.

\section{Eques punctatus Bloch and Schneider, 1801}

Eques punctatus Bloch and Schneider, 1801: 106, Pl. 3 (fig. 2) (Cuba). No types known.

Distribution: Bermuda, Florida, and Bahamas to Brazil.

\section{Genus Genyonemus Gill, 1861}

Genyonemus Gill, 1861c: 87. Neuter. Type species Leiostomus lineatus Ayres, 1855. Type by original designation.

Eastern Pacific in inshore shallow waters over sandy bottoms. Monotypic.

\section{Genyonemus lineatus (Ayres, 1855)}

Leiostomus lineatus Ayres, 1855: [2] col. 3 (San Francisco Bay, California, U.S.A.). Syntypes: ?USNM 368 (2).

Distribution: British Columbia to southern Baja California.

\section{Genus Isopisthus Gill, 1862}

Isopisthus Gill, 1862: 18 Masculine. Type species Ancylodon parvipinnis Cuvier, 1830. Type by original designation.

Amphi-American in inshore waters over sandy or muddy bottoms. Two species.

\section{Isopisthus parvipinnis (Cuvier, 1830)}

Ancylodon parvipinnis Cuvier in Cuvier and Valenciennes, 1830: 84, Pl. 105 (Cayenne, French Guiana). No types known. Syntypes: MNHN 0000-7453 (3).

Isopisthus affinis Steindachner, 1879b: 34 (Porto Alegre, Brazil). Syntypes: NMW 15190 (2). Species later illustrated and described in more detail in Steindachner 1879c: 43, Pl. 2 (fig. 2). Isopisthus harroweri Fowler, 1916: 402, fig. 3 (Colón, Panama). Holotype (unique): ANSP 45236.

Distribution: Western Atlantic: Costa Rica to southern Brazil. 


\section{Isopisthus altipinnis (Steindachner, 1866)}

Ancylodon altipinnis Steindachner, 1866a: 19 (West coast of South America). Holotype (unique): NMW 73479. Species first described in the abstract above, later illustrated and described in more detail in Steindachner 1866c: 209, Pl. 1 (fig. 3).

Isopisthus remifer Jordan and Gilbert, 1882a: 320 (Panama). Syntypes: ?BMNH 1903.5.15.166 (1); USNM 29169 (3), 29312 (1), 29324 (1), 29336 (1), 29429 (1).

Distribution: Baja California to Chile.

Comments: Pequeño (1989: 61) regarded Ancylodon altipinnis Steindachner, 1866 valid as Isopisthus altipinnis; synonymy follows Chao in Fischer et al. (1995: 1466). The Eschmeyer's Catalog of Fishes use Isopisthus remifer as the valid name for this species following recent literature (e.g. Chirichigno and Vélez, 1998: 314; Castro-Aguirre et al., 1999: 387; Nelson et al., 2004: 147; McMahan et al., 2013: 451; and Page et al., 2013: 152).

\section{Genus Johnius Bloch, 1793}

Johnius Bloch, 1793: 132. Masculine. Type species Johnius carutta Bloch, 1793. Type by subsequent designation. Type designated by Gill, 1861c: 85, also by Bleeker, 1876: 327.

Bola Hamilton, 1822: 75, 368. Feminine. Type species Bola coitor Hamilton, 1822. Type by subsequent designation. Type designated by Jordan and Thompson 1911: 244.

Apeches Gistel, 1848:IX. Masculine. Type species Johnius carutta Bloch, 1793. Type by being a replacement name. Unneeded replacement for Johnius Bloch, 1793.

Pseudomycterus Ogilby, 1908: 94. Masculine. Type species Pseudomycterus maccullochi Ogilby, 1908. Type by monotypy.

Wak Lin, 1938: 378. Masculine. Type species Bola coitor Hamilton, 1822. Type by being a replacement name. Unneeded substitute for Bola Hamilton, 1822.

Blythia Talwar, 1971b: 23. Feminine. Type species Umbrina dussumieri Valenciennes, 1833. Type by original designation. Objectively invalid; preoccupied by Blythia Theobald, 1868 in Colubridae, Serpentes.

Johnieops Lal Mohan, 1972b: 85 Masculine. Type species Sciaena osseus Day, 1876. Type by original designation.

Blythsciaena Talwar, 1975: 17. Feminine. Type species Umbrina dussumieri Valenciennes, 1833. Type by being a replacement name. Replacement for Blythia Talwar, 1971.

Coastal waters and estuaries of the Indo-West Pacific. Talwar and Joglekar (1992) recognized 10 species in the genus Johnius and 8 species in Johnieops; here there are 32 species.

\section{Johnius amblycephalus (Bleeker, 1855)}

Umbrina amblycephalus Bleeker, 1855c: 412 (Ambon Island, Moluccas Islands, Indonesia). Syntypes: RMNH 8289 (2).

Umbrina dussumieri Valenciennes in Cuvier and Valenciennes, 1833: 481 (Coromandel, India). Holotype (unique): MNHN 0000-9623.

Umbrina muelleri Klunzinger, 1879: 256 (Endeavour Strait, 1050' S, 142 15' E, Queensland, Australia). Syntypes: SMNS 2440 (2).

Distribution: Indo-West Pacific: east of Pakistan through the coastal waters of the Indian Ocean and the Indo-Australian Archipelago to the Philippines, New Guinea and to Repulse Bay, Queensland; through the South China Sea to Hainan, Taiwan, Hong Kong and Kwangtung. Replaced in the southwestern Indian Ocean by J. fuscolineatus (von Bonde, 1923).

\section{Johnius australis (Günther, 1880)}

? Sciaena novaehollandiae Steindachner, 1866e: 51 (Port Jackson, New South Wales, Australia). Holotype (unique): NMW 14618. Appeared first in the abstract as above, then in Steindachner 1866f: 445, Pl. 5 (fig. 2) as Sciaena (Corvina) novae hollandiae. 
Corvina australis Günther, 1880: 33 (Mary River near Tiaro, Queensland, Australia). Syntypes: (2) BMNH 1879.5.14.290 (1).

Corvina comes De Vis, 1884: 538 (Brisbane River, Queensland, Australia). Holotype (unique): QM I.949 (dry mount).

Pseudomycterus maccullochi Ogilby, 1908: 94 (Logan River, southern Queensland, Australia). Holotype (unique): QM I.1535 ex AFAQ.

Distribution: Known along the coasts of northern and northeastern Australia and New Guinea. Comments: Further investigations are needed to confirm Sciaena novaehollandiae Steindachner, 1866 as a senior synonym of Corvina australis Günther, 1880.

\section{Johnius belangerii (Cuvier, 1830)}

Corvina belangerii Cuvier in Cuvier and Valenciennes, 1830: 120 (Malabar, India). Syntypes: MNHN 0000-7613 (2, poor condition).

Corvina kuhlii Cuvier in Cuvierand Valenciennes, 1830: 121 (Labouane River, Java, Indonesia). No types known.

Corvina lobata Cuvier in Cuvier and Valenciennes, 1830: 122, Pl. 107 (Malabar, India). Syntypes: MNHN 0000-7587 (2).

Corvina nallakatshelee Richardson, 1846: 226 (Canton, China). Syntypes: (2) BMNH (1, half skin).

Sciaena (Corvina) nasus Steindachner, 1866d: 771, Pl. 15 (fig. 1) (Calcutta, India). Holotype (unique): NMW 76435.

Johnius fasciatus Chu, Lo and $\mathrm{Wu}, 1963$ : 23, figs. 11, 44, 70 (Kwanghai, Kwangtung Province, China). Holotype: SFC 56-20. Paratypes: SFC 56-19 (1), 56-74 (1).

Distribution: Arabian Sea to eastern Australia northward to southern China.

\section{Johnius borneensis (Bleeker, 1851)}

Otolithus borneensis Bleeker, 1851a: 268 (Bandjarmasin, Borneo, Indonesia). Holotype (unique): RMNH 5980.

Otolithus vogleri Bleeker, 1853: 253 (Benculen, Sumatra, Indonesia). Syntypes: RMNH 5979 (2).

Corvina goldmanni Bleeker, 1855a: 371 (Soengi Puan, [Soengi-Rass] Batjan, Indonesia). Holotype (unique): RMNH 5977. As goldmani in the Eschmeyer's Catalog of Fishes.

Corvina canina De Vis, 1884: 538 (Queensland, Australia). Holotype (unique): QM I.951 (dry, delicate condition).

Corvina papuensis Hase, 1914: 531, fig. 4 (Tami, northeastern New Guinea). Syntypes: ZMB 19138 (3).

Sciaena siamensis Hora, 1924: 487, fig. 4 (Singgora, Talé Sap, Thailand). Holotype (unique): ZSI.

Distribution: Persian Gulf east to Philippines and New Guinea, north to northern Vietnam and southern China.

Comments: In the taxonomic decisions of Sasaki $(1996$; 1999) the species of the subgenus Johnieops Sasaki (1999: 276) it was noted that: "Further studies may show that Philippines borneensis represents an undescribed species or necessitates resurrection of goldmani". Otolithus vogleri (with Corvina canina De Vis as synonym) has been regarded as a valid Johnius species in a number of works (Trewavas, 1977: 441; Allen and Swainston, 1988: 92; Randall, 1995: 231; Allen, 1997: 144; Carpenter et al., 1997: 190; Johnson, 1999: 737) and as a synonym of Johnius borneensis according to Sasaki (1996: 90; 1999: 275; 2001: 3154) and Kottelat (2013: 358).

\section{Johnius cantori Bleeker, 1874}

Johnius cantori Bleeker, 1874: 51 (Penang, Malaysia). Holotype (unique): BMNH 1860.3.19.223 (half skin, stuffed). 
Distribution: Known only from the holotype from Malaysia.

Comments: Species resurrected by Sasaki (1992).

Johnius carouna (Cuvier, 1830)

Corvina carouna Cuvier in Cuvier and Valenciennes, 1830: 125 (Malabar, India). Syntypes: MNHN 0000-7529 (1).

Sciaena glauca Day, 1876: 192, Pl. XLVI (fig. 2) (seas of India). Syntypes or Day specimens: AMS B.8236 (1, syntype), B.8237 (1, syntype); ZMB 9160 (1); ZSI 984-985 (2), 3025 (1, lost).

Distribution: Gulf of Thailand, Sumatra and Borneo; north to southern China, west to India.

Johnius carutta Bloch, 1793

Johnius carutta Bloch, 1793: 133, Pl. 356 (Hooghly Estuary near Diamond Harbor, India). Neotype: ZSI F6273/2.

Distribution: Pakistan, India, Sri Lanka, Malay Peninsula and Thailand.

\section{Johnius coitor (Hamilton, 1822)}

Bola coitor Hamilton, 1822: 75, 368, Pl. 27 (fig. 24) (Ganges River as far as Kanpur and Jumna River at Angra and at Visakhapatum, India). No types known.

Distribution: Bay of Bengal to Eastern Australia.

\section{Johnius distinctus (Tanaka, 1916)}

Sciaena distincta Tanaka, 1916: 26 (Nagasaki fish market, Japan). Holotype (unique): ZUMT 6811.

Pseudosciaena tingi Tang, 1937: 54, fig. 1 (Amoy; China). Holotype: ZMUA 14367. Paratypes: ZMUA 21421-24 (4).

Distribution: Japan and Taiwan, coasts of China south to Hong Kong.

Comments: Taxonomic decision of Sasaki and Amaoka (1989).

\section{Johnius dorsalis (Peters, 1855)}

Corvina dorsalis Peters, 1855: 434 (Quillimane, Mozambique, $18^{\circ}$ S). Syntypes: ZMB 896 (2).

Sciaena parva Gilchrist and Thompson, 1908: 183 (South Head Tugela River, north by west 4.5 mile, South Africa, depth 24 fathoms). Syntypes: (3) ?BMNH 1927.12.6.25 ex SAM (1), SAM 9961 (3).

Distribution: East African coast from Kenya to South Africa and Madagascar.

Johnius dussumieri (Cuvier, 1830)

Corvina dussumieri Cuvier in Cuvier and Valenciennes, 1830: 119 (Malabar, India). Syntypes: MNHN 0000-7614 (1).

Sciaena osseus Day, 1876: 193, Pl. 46 (fig. 3) (Malabar coast of India). Lectotype: ZSI F1340 (figured). Paralectotypes: AMS B.8249 (1).

Distribution: Pakistan to Malay Peninsula and northern Australia; records from East Africa are misidentification with $J$. dorsalis (Peters).

Comments: Taxonomic decision of Sasaki (1996; 1997; 1999). Johnius dussumieri has been considered a valid species by many authors (Daget and Trewavas, 1984; Talwar and Joglekar, 1992). Sciaena ossea is treated as valid by de Bruin et al. (1995).

\section{Johnius elongatus Lal Mohan, 1976}

Johnius elongatus Lal Mohan, 1976: 19, fig. 1 (Veraval, India, Arabian Sea). Holotype: FMRI 187. Paratypes: FMRI 188 (7).

Distribution: Arabian Sea, west coast of India and Sri Lanka. 
Johnius fuscolineatus (von Bonde, 1923)

Umbrina striata Gilchrist and Thompson, 1908: 181 (Natal, South Africa; objectively invalid, nomen preoccupatum, preoccupied by Umbrina striata Boulenger, 1887). Holotype (unique): whereabouts unknown.

Umbrina fuscolineata von Bonde, 1923: 15, Pl. 4 (Natal, South Africa, Pickle sta. 180, 28 fms). Holotype (unique): whereabouts unknown.

Distribution: Coasts of Mozambique, South Africa and Madagascar.

Comments: Umbrina fuscolineata was removed from synonymy with $J$. amblycephalus by Sasaki (1997).

\section{Johnius gangeticus Talwar, 1991}

Johnius novaehollandiae (nec Steindachner) Babu Rao and Sinha, 1963.

Johnius gangeticus Talwarin Talwar and Jhingran 1991: 859, fig. 261 (Ganges River at Allahabad, Uttar Pradesh, India). Holotype: ZSI FF 2605. Paratypes: ZSI FF 2606 (26).

Distribution: Ganges River, including its estuary in India.

\section{Johnius grypotus (Richardson, 1846)}

Corvina grypota Richardson, 1846: 225 (Canton, China). No types known.

Distribution: West Pacific: along the coasts of China and Taiwan, from the Gulf of Pohai to Hainan.

Comments: Resurrected species (Sasaki 1990).

\section{Johnius heterolepis Bleeker, 1873}

Johnius heterolepis Bleeker, 1873: 456, Pl. 21 (upper figure) (Suriname). Syntypes: RMNH 6042 (2).

Distribution: Indo-West Pacific: Gulf of Thailand, Malaysia and Indonesia (type locality is evidently erroneous).

Johnius hypostoma (Bleeker, 1853)

Corvina hypostoma Bleeker, 1853: 499 (Padang, Sumatra, Indonesia). Syntypes: (3) 5976 (3 of 5).

Distribution: Sumatra.

Johnius laevis Sasaki and Kailola, 1991

Johnius (Johnius) laevis Sasaki and Kailola, 1991: 119, figs. 1-2 (off Port Essington, $11^{\circ} 07^{\prime}$ S, $131^{\circ} 44^{\prime}$ E, Northern Territory, Australia, depth 45 meters). Holotype: NTM S.11452-012. Paratypes: AMS I.20402-023 (1), I.21632-002 (1); CSIRO A 3131 (1), CA 2797 (1); NTM S.11452-002 (28).

Distribution: Northern Australia, New Guinea.

\section{Johnius latifrons Sasaki, 1992}

Johnius (Johnius) latifrons Sasaki, 1992: 195, figs. 2C, 4 (Songkhla, Thailand). Holotype: NSMT-P 44942. Paratypes: AMS I.21033-006 (1), I.28995-008 (1); BPBM 18605 (1), 18620 (1); HUMZ 101707-08 (1, 1); NSMT-P 44940 (2).

Distribution: Thailand and Indonesia.

\section{Johnius macropterus (Bleeker, 1853)}

Umbrina macropterus Bleeker, 1853: 254 (Priaman, Sumatra, Indonesia). Syntypes: (2) RMNH 5993 (now 3).

Distribution: India and Sri Lanka eastward to Thailand and Malaysia, southward to New Guinea.

\section{Johnius macrorhynus (Mohan, 1976)}

Johnieops macrorhynus Mohan, 1976: 20, fig. 2 (Mumbai, India). Holotype: FMRI 189. Paratypes: FMRI 190/1-19 (19). 
Distribution: Arabian Sea, Andaman Sea, Singapore.

Johnius majan Iwatsuki, Jawad and Al-Mamry, 2012

Johnius (Johnius) majan Iwatsuki, Jawad and Al-Mamry, 2012: 152, figs. 1-2 (Madrakah, southern Oman, $\left.18^{\circ} 58^{\prime} 09.17^{\prime \prime} \mathrm{N}, 5^{\circ} 47^{\prime} 10.41^{\prime \prime} \mathrm{E}\right)$. Holotype: MUFS 33607. Paratypes: BSKU 105320-21 (2); MUFS 33727 (1), 33728 (1); OMMSFC 1074-1076 (3).

Distribution: Northern Indian Ocean: Arabian Peninsula and Gulf of Oman.

\section{Johnius mannarensis Lal Mohan, 1971}

Johnius mannarensis Lal Mohan, 1971: 320, fig. 1 (Off Pamban, Gulf of Mannar, India, depth 15 fathoms). Holotype: FMRI 149. Paratypes: FMRI 150A-B (2).

Distribution: Gulf of Mannar.

Comments: Removed from synonymy with J. macropterus by Sasaki (1994).

\section{Johnius novaeguineae (Nichols, 1950)}

Collichthys novaeguineae Nichols, 1950: 2 (Murauke River, New Guinea). Holotype: AMNH 18729. Paratypes: AMNH 17568 (4).

Distribution: Lower Rivers of southern New Guinea and northern Australia.

\section{Johnius pacificus Hardenberg, 1941}

Johnius pacificus Hardenberg, 1941: 229 (mouth of the river Mamberamo, northern New Guinea). Holotype (unique): not researched.

Distribution: Northern Pacific coast of New Guinea.

\section{Johnius philippinus Sasaki, 1999}

Johnius (Johnieops) philippinus Sasaki, 1999: 271, figs. 1-2 (Agdao fish market, Davao City, Mindanao, Philippines). Holotype: NSMT-P 55912. Paratypes: BSKU 86010-14 (10, 9, 10, 2 , 1); NSMT-P 55913 (9).

Distribution: Known only from the Davao Gulf, Mindanao, Philippines.

\section{Johnius plagiostoma (Bleeker, 1849)}

Corvina plagiostoma Bleeker, 1849a: 10 (Madura Straits near Surabaya and Kammal, Java, Indonesia). Syntypes: RMNH 5984 (2).

Distribution: Java westward to Burma and Bangladesh; Borneo and Vietnam.

\section{Johnius sina (Cuvier, 1830)}

Corvina sina Cuvier in Cuvier and Valenciennes, 1830: 122 (Puducherry and Malabar, India; Japan). Syntypes: MNHN 0000-7521 (2), 7633 (1, dry), B-2648 ex MNHN 0000-7521 (1, poor condition); ZMB 892 (1), 8737 (dry) Japan.

Wak menoni Talwar and Joglekar, 1970a: 33, fig. 1 (Diamond harbor, Hooghly Estuary, West Bengal, India). Holotype: ZSI F6136/2. Paratypes: ZSI F6137/2 (7).

Distribution: Indo-west Pacific: Natal to India and Malay Peninsula (not in the Red Sea)

Comments: Regarded either as valid as Johnius sina (Cuvier, 1830) (Trewavas, 1977: 439; Daget and Trewavas, 1986: 334; Krishnan and Mishra, 1993: 228; Randall, 1995: 230; Khan, 2003: 9) or as a synonym of Johnius dussumieri (Cuvier, 1830) (Sasaki, 1996: 92; Carpenter et al., 1997: 189; Sasaki, 1999: 274).

\section{Johnius taiwanensis Chao, Chang, Chen, Guo, Lin, Liou, Shen and Liu, 2019}

Johnius taiwanensis Chao, Chang, Chen, Guo, Lin, Liou, Shen and Liu, 2019: 262, figs. 3b-9 (Jiangjun Port, Tainan, Taiwan). Holotype: NMMBP 23063. Paratypes: CAS 244474 (1) 244631 (2), MCZ 172776 (1), 172798 (2); NMMBP 8464 (1), 19365 (3), 19373 (9), 23065 (2) 23067 (1), 23070 (1), 23580 (1), 24335 (1), 24925 (2), 25882 (3), 27962 (2), 31330, (3), 31331 (1) 23023 (2), 24237 (4, 27621 (1), 27964 (4), 27965 (3), 30143 (1); USNM 244361 (2).

Distribution: Taiwan Strait, China and Taiwan, western Pacific. 


\section{Johnius trachycephalus (Bleeker, 1851)}

Corvina trachycephalus Bleeker, 1851a: 269 (Bandjarmasin, Borneo, Indonesia). Holotype (unique): RMNH 5973 ?5975 (1 of 4).

Otolithoides siamensis Fowler, 1934: 153, fig. 121 (Bangkok, Thailand). Holotype (unique): ANSP 60171.

Distribution: Sumatra, Borneo, Thailand and South China Sea.

\section{Johnius trewavasae Sasaki, 1992}

Johnius trewavasae Sasaki, 1992b: 191, figs. 1, 2A (Taiwan Strait). Holotype: HUMZ 109504. Paratypes: ANSP 52863 (1), 53478 (2); CAS-SU 60859 (1), 60870 (1), 60918 (1), 61045 (3, 2 cands), 61080 (2), 61484 (2); HUMZ 109501 (1).

Distribution: West Pacific: South China Sea, East China Sea.

\section{Johnius weberi Hardenberg, 1936}

Johnius weberi Hardenberg, 1936: 251 (Telok Pekadai, Kapaus River, Borneo; mouth of Pekadai; mouth of Peniti River; Pedang Tukar Bay). Syntypes: (6) ZMA 113119 (2), 113120 (2).

Distribution: Indo-Australian Archipelago: reported from Thailand to Vietnam, Hong Kong, Indonesia and Papua New Guinea.

\section{Genus Kathala Lal Mohan, 1969}

Kathala Lal Mohan, 1969: 295. Masculine. Type species Corvina axillaris Cuvier, 1830. Type by original designation.

Dhoma Talwar and Joglekar, 1970: 361. Feminine. Type species Corvina axillaris Cuvier, 1830. Type by original designation.

Northern Indian Ocean in shallow coastal waters. Monotypic.

Comments: The article of Talwar and Joglekar was prepared for a volume expected for December 1968 but was actually issued on July 1970. The authors cannot be aware of the genus name Kathala erected by Lal Mohan (1969) for the same nominal species.

\section{Kathala axillaris (Cuvier, 1830)}

Corvina axillaris Cuvier in Cuvier and Valenciennes, 1830: 113 (Malabar, India). Syntypes: MNHN 0000-7682 (2) Malabar, 0000-7484 (4, poor condition) Puducherry, 0000-5314 (1)

Malabar.

Distribution: Persian Gulf, India, Sri Lanka.

\section{Genus Larimichthys Jordan and Starks, 1905}

Larimichthys Jordan and Starks, 1905: 204. Masculine. Type species Larimichthys rathbunae Jordan and Starks, 1905. Type by monotypy.

Othonias Jordan and Thompson, 1911: 244. Masculine. Subgenus of Sciaena, type species Sciaena manchurica Jordan and Thompson, 1911. Type by original designation.

West Pacific; three species.

\section{Larimichthys crocea (Richardson, 1846)}

Sciaena crocea Richardson, 1846: 224 (Canton, China). Holotype: BMNH 1968.3.11.9.

Pseudosciaena amblyceps Bleeker, 1863a: 142 (Amoy, China). Lectotype: RMNH 735. Paralectotypes: RMNH 24929 ex RMNH 735 (5). Lectotype selected by Trewavas 1977: 393. Pseudosciaena undovittata Jordan and Seale, 1905b: 11, Pl. 6 (Market at Hong Kong, China). Holotype (unique): CAS-SU 9065.

Distribution: South China Sea, East China Sea, Yellow Sea, Bohai Sea.

Comments: Often valid as Pseudosciaena crocea (e.g. Trewavas, 1977: 392; Okamura in Masuda et al., 1984: 162). 


\section{Larimichthys pamoides (Munro, 1964)}

Pseudosciaena pamoides Munro, 1964: 176, fig. 18 (Mouth of Fly River, Gulf of Papua, Papua New Guinea). Holotype (unique): CSIRO A 1838.

Distribution: Along coasts of northwestern Australia and southern Papua New Guinea.

\section{Larimichthys polyactis (Bleeker, 1877)}

? Sciaena chuanchua Basilewsky, 1855: 221 (in Mari orientali et sinu Tschiliensi [= China Sea and Gulf of China]). No types at ZIN.

Pseudosciaena polyactis Bleeker, 1877: 2 (Shanghai, China). Holotype (unique): ZMH 2408. Described in more detail in Bleeker 1879: 5, Pl. 1 (fig. 1).

Larimichthys rathbunae Jordan and Starks, 1905: 204, fig. 8 (Coast of Korea). Holotype (unique): USNM 45299.

Sciaena manchurica Jordan and Thompson, 1911: 255, fig. 3 (Port Arthur, Manchuria, China). Holotype: USNM 67330. Paratypes: CAS-SU 9989 (7).

Sciaena ogiwara Nichols, 1913: 180, fig. 2 (Shimonoseki, Yamaguchi, Honshu, Japan). Holotype (unique): AMNH 3958.

Othonias brevirostris Wang, 1935: 452, fig. 34 (Chefoo, Shantung Province, China). Holotype (unique): SSCN 10333.

Sciaena aurea Tanaka, 1916: 27 (Nagasaki fish market, Japan). Syntypes: ZUMT 6818-19 (2, lost).

Distribution: Japan and China Sea.

\section{Genus Larimus Cuvier, 1830}

Larimus Cuvier in Cuvier and Valenciennes, 1830: 145. Masculine. Type species Larimus breviceps Cuvier, 1830. Type by monotypy.

Amblyscion Gill, 1863b: 165. Masculine. Type species Amblyscion argenteus Gill, 1863. Type by monotypy.

Monosira Poey, 1881: 326. Feminine. Type species Monosira stahli Poey, 1881. Type by monotypy.

Western Atlantic (two species) and eastern Pacific in tropical estuarine and inshore open waters over sandy and muddy bottoms. Seven species.

\section{Larimus acclivis Jordan and Bristol, 1898}

Larimus acclivis Jordan and Bristol in Jordan and Evermann, 1898: 1422 (San Juan Lagoon, Sonora, Pacific coast of Mexico). Syntypes: CAS-SU 45 (3).

Distribution: Gulf of California to northern Peru.

\section{Larimus argenteus (Gill, 1863)}

Amblyscion argenteus Gill, 1863b: 165 (Western coast of Central America). Holotype (unique): USNM lost.

Distribution: Gulf of California to northern Peru. Inhabits coastal waters and lagoons.

\section{Larimus breviceps Cuvier, 1830}

Larimus breviceps Cuvier in Cuvier and Valenciennes, 1830: 146, Pl. 111 (Brazil and Dominican Republic). Syntypes: MNHN 0000-7578 (1) Dominican Republic, 0000-7636 (1) Brazil.

Monosirastahli Poey, 1881: 326, Pl. 6 (Puerto Rico). No types known.

Distribution: Antilles to Brazil.

\section{Larimus effulgens Gilbert, 1898}

Larimus effulgens Gilbert in Jordan and Evermann, 1898: 1421 (Pacific Panama). Lectotype: USNM (not located). Paralectotypes: BMNH 1903.5.15.123-126 from Jordan (4), CAS-SU 5520 (1). SU 5520 as holotype in Böhlke 1953: 78. Also appeared as Gilbert in Gilbert and 
Starks 1904: 123, Pl. 16 (fig. 33), with lectotype established by use of "type specimen" in caption to figure 33 .

Distribution: Gulf of California to northern Peru. Inhabits coastal waters and lagoons.

\section{Larimus fasciatus Holbrook, 1855}

Larimus fasciatus Holbrook, 1855: 153, Pl. XXII (fig. 1) (South Carolina). No types known.

Distribution: Massachusetts to Texas, except southern Florida.

\section{Larimus gulosus Hildebrand, 1946}

Larimus gulosus Hildebrand, 1946: 298, fig. 64 (Lobos de Tierra I., Peru). Holotype (unique): USNM 77694.

Distribution: Off Peru and Chile.

Comments: Larimus gulosus Hildebrand is a probable synonym of L. pacificus according to Chao (1995: 1470), but a valid species according to Pequeño (1989: 61), Chirichigno and Vélez (1998: 328).

\section{Larimus pacificus Jordan and Bollman, 1890}

Larimus pacificus Jordan and Bollman, 1890: 161 (Panama Bay, ca. 5 nautical mile northeast of Isal Otoque, $8^{\circ} 38^{\prime} \mathrm{N}, 79^{\circ} 31^{\prime} 30^{\prime \prime} \mathrm{W}$, Albatross sta. 2802, 16 fathoms). Holotype (unique): USNM 41168.

Distribution: Gulf of California to Chile. Very common in Ecuador. Inhabits coastal waters up to a depth of $50 \mathrm{~m}$.

\section{Genus Leiostomus Lacepède, 1802}

Leiostomus Lacepède, 1802: 438. Masculine. Type species Leiostomus xanthurus Lacepède, 1802. Type by monotypy.

Liostomus Agassiz 1846: 204, 212 (unjustified emendation).

Western Atlantic in estuarine and inshore waters. Monotypic.

\section{Leiostomus xanthurus Lacepède, 1802}

Leiostomus xanthurus Lacepède, 1802: 438, 439, Pl. 10 (fig. 1) (Carolina, U.S.A.). Syntypes: MNHN 0000-7599 (2).

Mugil obliquus Mitchill, 1814: 16 (New York, U.S.A.). No types known.

Labrus obliquus Mitchill, 1815: 405 (New York, U.S.A.). No types known.

Johnius humeralis Cuvier, 1829: 173 (New York, U.S.A.). No types known.

Sciaena multifasciata Lesueur, 1822: 255 (Eastern Florida, U.S.A.). Holotype (unique): whereabouts unknown.

Leiostomus humeralis Cuvier in Cuvier and Valenciennes, 1830: 141, Pl. 110 (Philadelphia and New York, U.S.A.). Syntypes: MNHN 0000-7501 (1), 0000-7593 (1), 0000-7597 (1), 0000-9733 (1, dry); Philadelphia specimen lost; SMF 1437 [ex MNHN in 1830] (1) New York.

Distribution: Massachusetts to northern Mexico, except southern Florida.

\section{Genus Lonchurus Bloch, 1793}

Lonchurus Bloch, 1793: 143. Masculine. Type species Lonchurus barbatus Bloch, 1793. Type by monotypy.

Lonchiurus Bloch, 1797: 120 (misspelling or unjustified emendation)

Western Atlantic in coastal and estuarine waters over sandy or muddy bottoms. Monotypic.

\section{Lonchurus lanceolatus (Bloch, 1788)}

Perca lanceolata Bloch, 1788: 383 (Suriname). Holotype (unique): ZMB 927.

Lonchurus barbatus Bloch, 1793: 144, Pl. 360 (Suriname). Holotype (unique): ZMB 927. As

Lonchiurus barbatus Bloch, 1797: 120. P1. 360. 
Lonchurus depressus Bloch and Schneider, 1801: 102 (Suriname). Syntypes: ZMB 926 (2).

Lonchurus dianema Lacepède, 1801: 413, 414 (Suriname). Nomen novum, unneeded replacement name for Lonchurus barbatus Bloch, 1788.

Distribution: Southwestern Atlantic.

\section{Genus Macrodon Schinz, 1822}

Ancylodon Cloquet in Cuvier, 1816a: 44. Masculine. Type species Ancylodon surinamense Cuvier, 1816). Objectively invalid; preoccupied by Ancylodon Illiger, 1811 in Ziphiidae, Cetacea, Mammalia.

Ancylodon Oken (ex Cuvier) 1817: 1182. Masculine. Type species Lonchurus ancylodon Bloch and Schneider, 1801. Type apparently by subsequent absolute tautonymy. Objectively invalid; preoccupied by Ancylodon Illiger 1811 in Ziphiidae, Cetacea, Mammalia.

Macrodon Schinz, 1822: 482. Masculine. Type species Lonchurus ancylodon Bloch and Schneider, 1801. Type by being a replacement name. Replacement for Ancylodon Cloquet, 1816.

Nomalus Gistel, 1848:VIII. Feminine. Lonchurus ancylodon Bloch and Schneider, 1801. Type by being a replacement name. Unneeded replacement for Ancylodon Cloquet, 1816 or Oken, 1817 already replaced by Macrodon Schinz, 1822.

Sagenichthys Berg, 1895: 52. Masculine. Type species Lonchurus ancylodon Bloch and Schneider, 1801. Type by being a replacement name. Unneeded replacement for Ancylodon Bosc 1816 or Oken (Cuvier) 1817, already replaced by Macrodon Schinz, 1822 and Nomalus Gistel, 1848.

Amphi-American in inshore waters over sandy or muddy bottoms. Three species.

\section{Macrodon ancylodon (Bloch and Schneider, 1801)}

Lonchurus ancylodon Bloch and Schneider, 1801: 102, Pl. 25 (Suriname). Syntypes: ZMB 868 (4).

Ancylodon surinamense Cloquet in Cuvier, 1816a: 45 (Suriname). No types known. Nomen novum, unneeded replacement name for Lonchurus ancylodon Bloch and Schneider, 1801.

Ancylodon jaculidens Cuvier in Cuvier and Valenciennes, 1830: 81 (Cayenne, French Guiana). Syntypes: MNHN 0000-7451 (1), 0000-7454 (1).

Stellifer mercatoris Delsman, 1941: 71, fig. 10 (Mouth of Amazon River, $1^{\circ} 06^{\prime} \mathrm{N}, 47^{\circ} 53^{\prime} \mathrm{W}$, Brazil, depth 20-30 fathoms). Syntypes: IRSNB 56 (7).

Distribution: Venezuela to Argentina.

\section{Macrodon atricauda (Günther, 1880)}

Ancylodon atricauda Günther, 1880: 12 (Río de la Plata mouth, Challenger station 321, depth 13 fathoms). Holotype (unique): BMNH 1879.5.14.258.

Percamollis Larrañaga, 1923: 380, 385 (Uruguay). No types known.

Distribution: Brazil to Argentina.

Comments: Resurrected from synonymy with Macrodon ancylodon by Carvalho-Filho et al. (2010).

\section{Macrodon mordax (Gilbert and Stark, 1904)}

Sagenichthys mordax Jordan and Starks, 1904: 121, Pl. 16 (fig. 32) (Panama Bay, Pacific Panama). Holotype: CAS-SU 6809. Paratypes: ?BMNH 1903.5.15.163-165 from Jordan (3), CAS-SU 6656 (4), USNM 50401 (1), ZMB 15967 ex SU (1).

Distribution: Panama to Colombia. Inhabits coastal waters, bays and estuaries.

\section{Genus Macrospinosa Lal Mohan, 1969}

Macrospinosa Lal Mohan, 1969: 295. Feminine. Type species Bola cuja Hamilton, 1822. Type by original designation. 
Cantor Talwar, 1970: 68. Masculine. Type species Bola cuja Hamilton, 1822. Type by original designation.

India. Monotypic.

\section{Macrospinosa cuja (Hamilton, 1822)}

?Johnius serratus Bloch and Schneider, 1801: 76 (Tranquebar, India). Holotype (unique): ZMB 8723 (dry, missing).

Bola cuja Hamilton, 1822: 81, 369, Pl. 12 (fig. 27) (Ganges River estuaries, India). No types known.

Sciaenoides asper Blyth, 1860: 140 (mouth of the Gangetic Rivers, India). No types known.

Distribution: Ganges River estuary (India).

Comments: Johnius serratus Bloch and Schneider is a doubtful species, placement uncertain (Trewavas, 1977: 448).

\section{Genus Megalonibea Chu, Lo and Wu, 1963}

Megalonibea Chu, Lo and Wu, 1963: 34. Feminine. Type species Megalonibea fusca Chu, Lo and $\mathrm{Wu}, 1963$. Type by original designation.

Indo-West Pacific in coastal waters over muddy bottoms; monotypic.

\section{Megalonibea fusca Chu, Lo and Wu, 1963}

Megalonibea fusca Chu, Lo and Wu, 1963: 35 (English p. 90, figs. 19, 48, 74) (Shanghai fish market, China). Holotype (unique): SFC ? now at Amoy.

Distribution: East China Sea.

\section{Genus Menticirrhus Gill, 1861}

Menticirrhus Gill, 1861c: 86. Masculine. Type species Perca alburnus Linnaeus, 1766. Type by original designation.

Cirrimens Gill, 1862: 17. Feminine. Type species Umbrina ophicephala Jenyns, 1840. Type by original designation.

Umbrula Jordan and Eigenmann, 1889: 423, 426. Feminine. Subgenus of Menticirrhus, type species Umbrina littoralis Holbrook, 1847. Type by original designation.

Amphi-American in shallow coastal waters with sandy bottoms; three species in the western Atlantic and six species in the eastern Pacific.

\section{Menticirrhus americanus (Linnaeus, 1758)}

Cyprinus americanus Linnaeus, 1758: 321 (America Carolinas). No types known. Based on a figure and short account of Catesby 1743: 12.

Perca alburnus Linnaeus, 1766: 482 (Carolina [=South Carolina, U.S.A.]). Syntypes: Linn. Soc. London 111 (left half-skin).

Umbrina martinicensis Cuvier in Cuvier and Valenciennes, 1830: 186 (Martinique Island, West Indies). Syntypes: MNHN 0000-7498 (1).

Umbrina gracilis Cuvier in Cuvier and Valenciennes, 1830: 189 (Brazil). Lectotype: MNHN 0044 (dry). Paralectotypes: MNHN 0000-9622 (1). Lectotype selected by Chao 1978: 30.

Umbrina arenata Cuvier in Cuvier and Valenciennes, 1830: 190 (Brazil). Syntypes: MNHN 0000-7472 (1, dry), 0000-7500 (1).

Umbrina phalaena Girard, 1858b: 167 (Indianola and Brazos Santiago, Texas). Syntypes: MCZ 482 ex USNM 620 (1); USNM 619 (1, not found in 1993), 620 (1), 128273 ex USNM 620 (2), ?154721 ?ex USNM 620 (1).

Umbrina januaria Steindachner, 1876a: 170 (Rio de Janeiro, Brazil). Syntypes: (several) not yet found at NMW.

Distribution: New York to Texas and Bay of Campeche to Argentina; rare in Antilles and southern Florida. 
Comments: Cyprinus americanus Linnaeus, 1766 is a different species: the name is invalid and synonym of Notemigonus crysoleucas (Mitchill 1814), family Leuciscidae.

\section{Menticirrhus elongatus (Günther, 1864)}

Umbrina elongata Günther, 1864: 148 (Chiapam, Guatemala). Holotype (unique): BMNH 1864.1.26.243.

Menticirrhus rostratus Hildebrand, 1946: 265, fig. 58 (Paita Bay, Peru). Holotype: USNM 128002. Paratypes: USNM 128003 (1).

Distribution: Baja California to southern Peru.

\section{Menticirrhus littoralis (Holbrook, 1847)}

Umbrina littoralis Holbrook, 1847: 10, Pl. 1 (South Carolina, U.S.A.). Holotype: ANSP 11576. Also appeared in Holbrook 1855: 142, Pl. 20 (fig. 2) and in the second edition, 1860: 144, Pl. 20 (fig. 2).

Distribution: Chesapeake Bay to northern Gulf of Mexico and Brazil, except southern Florida.

\section{Menticirrhus nasus (Günther, 1868)}

Umbrina nasus Günther, 1868: 426, figs. (Pacific Panama). Holotype (unique): BMNH 1867.9.23.17.

Menticirrhus simus Jordan and Eigenmann, 1889: 427 (Mazatlán, Sinaloa, western Mexico; Pacific Panama). Holotype: USNM 28292.

Distribution: Baja California to northern Peru.

\section{Menticirrhus ophicephalus (Jenyns, 1840)}

Umbrina ophicephala Jenyns, 1840: 45 (Coquimbo, Chile). Syntypes: BMNH (2).

Menticirrhus cokeri Evermann and Radcliffe, 1917: 107, Pl. 10 (fig. 2) (Ancon, Peru). Holotype: USNM 77533. Paratypes: (1).

Distribution: Chile and Peru.

\section{Menticirrhus paitensis Hildebrand, 1946}

Menticirrhus paitensis Hildebrand, 1946: 263, fig. 57 (South shore of Paita Bay, Peru). Holotype (unique): USNM 128004.

Distribution: Baja California to southern Peru.

\section{Menticirrhus panamensis (Steindachner, 1876)}

Umbrina panamensis Steindachner, 1876b: 559, Pl. 9 (Pacific Panama). Syntypes: (several) MCZ 520 (1), 8623 (1), 8624 (2); NMW 82663 (1); USNM 153615 ex MCZ 8624 (1).

Menticirrhus agassizi Jordan in Jordan and Eigenmann, 1889: 429 (Caldera, Chile). Holotype (unique): MCZ 99493 ex MCZ 8603A.

Distribution: Baja California to Chile.

\section{Menticirrhus saxatilis (Bloch and Schneider, 1801)}

Johnius saxatilis Bloch and Schneider, 1801: 75 (New York, U.S.A.). Holotype (unique): ZMB 8792 (stuffed).

Sciaena nebulosa Mitchill, 1815: 408, Pl. 3 (New York, U.S.A.). No types known. Objectively invalid, nomen preoccupatum, preoccupied by Sciaena nebulosa Forsskål, 1775, Kuhliidae;

Menticirrhus focaliger Ginsburg, 1952: 97, fig. 8 (St. Joseph Bay, near its entrance, Florida, U.S.A.). Holotype: USNM 144161. Paratypes: USNM 144162-63 (19, 5); FMNH 50102 (2). Menticirrhus atlanticus McFarland, 1963: 98 (Mustang Id, Texas)

Distribution: Massachusetts to southern Florida and Gulf of Mexico to Yucatan.

\section{Menticirrhus undulatus (Girard, 1854)}

Umbrina undulata Girard, 1854: 148 (San Diego, California, U.S.A.). Syntypes: USNM 370 (1). 
Distribution: Punta Concepcion to Gulf of California.

\section{Genus Micropogonias Bonaparte, 1831}

Micropogon Cuvier in Cuvier and Valenciennes, 1830: 213. Masculine. Type species Micropogon lineatus Cuvier, 1830. Type by subsequent designation. Type designated by Bleeker, 1876: 326 as $M$. costatus DeKay not an included species = M. lineatus. Objectively invalid; preoccupied by Micropogon Boie, 1826 in Bucconidae, Piciformes, Aves.

Micropogonias Bonaparte, 1831: 170. Masculine. Type species Micropogon lineatus Cuvier 1830. Type by being a replacement name. Replacement for Micropogon Cuvier 1830.

The number of species varies from 5 according to Chao (1978) to 7 (see for example Pequeño, 1989 and Allen and Robertson, 1994). Six species are listed here.

\section{Micropogonias altipinnis (Günther, 1864)}

Micropogon altipinnis Günther, 1864: 149 (Chiapam, Guatemala; San José, Guatemala). Syntypes: (2) BMNH 1864.1.26.325 (1) San José.

Distribution: Gulf of California to Peru.

\section{Micropogonias cevegei (Cervigón, 1982)}

Pachypops cevegei Cervigón, 1982: 237, figs. 17-18 (Caño Mánamo, near Tucupita, Venezuela). Holotype: FCLR 641. Paratypes: FCLR 642 (1), 643 (1).

Distribution: Orinoco River, Venezuela.

Comments: Placed in Micropogonias by Casatti (2002).

\section{Micropogonias ectenes (Jordan and Gilbert, 1882)}

Micropogon ectenes Jordan and Gilbert, 1882d: 355 (Mazatlán, Sinaloa, w. Mexico). Syntypes: USNM 28295 (1), 28336 (1), 28361 (1), 29538 (1).

Distribution: Gulf of California, Mexico.

Comments: Based on mitochondrial DNA analysis, Sánchez-Pinedo et al. (2017: 136) proposed the species be a junior synonym of $M$. altipinnis. This decision needs to be substantiated by a more comprehensive morphological analysis.

\section{Micropogonias furnieri (Desmarest, 1823)}

Umbrina furnieri Desmarest, 1823: 182, P1. 2 (fig. 3) (Havana, Cuba). Syntypes: MNHN 0000-4968 (1), 0000-7577 (2).

Sciaena opercularis Quoy and Gaimard, 1825: 347 (Rio de Janeiro, Brazil). Holotype (unique): not found at MNHN.

Micropogon argenteus Cuvier in Cuvier and Valenciennes, 1830: 218 (Suriname). Holotype (unique): RMNH 685.

Micropogon lineatus Cuvier in Cuvier and Valenciennes, 1830: 215, Pl. 119 (Havana, Cuba). Lectotype: MNHN 0000-4968. Paralectotypes: MNHN 0000-7457 (2) New York, 0000-7577 (2) Havana, 0000-7459 (1) Montevideo; 1987-154 ex MNHN 0000-6459 (1) Montevideo, 1987-0153 ex MNHN 0000-7457 (1). Lectotype selected by Chao 1978: 32.

Corvina crawfordi Regan, 1903: 627 (Montevideo, Uruguay). Holotype (unique): BMNH 1903.10.16.7.

Micropogon patagonensis MacDonagh, 1931: 409 (Richo de San Blas, Argentina). Holotype: MLP.

Micropogon barretoi MacDonagh, 1934: 70 (Río La Plata, Bay of Samborombón, Punta Piedras, Argentina). Holotype (unique): MLP 12.VII.33.15.

Ophioscion woodwardi Fowler, 1937: 311, fig. (Port-au-Prince, Haiti, West Indies). Holotype: ANSP 68257. Paratypes: ANSP 68258-60 (3).

Micropogon manni Moreno, 1970: 5, fig. 1 (Vichuquén and Torca lagoons, near Llico, Curicó Prov., Chile, $34^{\circ} 45^{\prime}$ S, $72^{\circ} 07^{\prime}$ W). Holotype: MNHNC P.5622. Paratypes: MNHNC P.5623$27(1,1,1,1,1)$, DBFFEUCS (3). 
Distribution: Western Atlantic: Greater Antilles and from Costa Rica to Argentina. Eastern Pacific: off Chile.

Comments: Sciaena opercularis is reported as valid by Bauchot and Desoutter (1987); Chao (1978) considered this species a synonym of M. undulatus, whereas it is synonym of Micropogonias furnieri according to Cousseau and Perrotta (2000: 112). Again, valid as Micropogon opercularis by López et al. (2002: 65). Micropogon manni was regarded as valid by Pequeño (1989: 62), but a synonym of Micropogonias furnieri according to Kong and Valdés (1990: 50) and Meléndez et al. (1993: 148). However, M. furnieri is a western Atlantic species.

\section{Micropogonias megalops (Gilbert, 1890)}

Micropogon megalops Gilbert, 1890: 64 (Gulf of California, Mexico, 3047'00" N, $113^{\circ} 13^{\prime} 00^{\prime \prime} \mathrm{W}$, Albatross sta. 3021, 14 fms). Syntypes: (4) USNM 46503 (2).

Distribution: Gulf of California to Acapulco, Mexico.

Comments: Based on mitochondrial DNA analysis, Sánchez-Pinedo et al. (2017: 136) proposed Micropogon megalops be a junior synonym of M. altipinnis.

\section{Micropogonias undulatus (Linnaeus, 1766)}

Perca undulata Linnaeus, 1766: 483 (Carolina South Carolina and Chesapeake Bay, Virginia, U.S.A.) Syntypes: LS 112 (right half-skin), 113 (left half-skin).

Sciaena croker Lacepède, 1802: 309, 314 (Carolinas, U.S.A.). No types known.

Bodianus costatus Mitchill, 1815: 417 (New York, U.S.A.). No types known.

Distribution: Massachusetts and northern Gulf of Mexico to Brazil and Argentina.

\section{Genus Miichthys Lin, 1938}

Miichthys Lin, 1938: 165. Masculine. Type species Sciaena miiuy Basilewsky, 1855. Type by original designation (also monotypic).

West Pacific in coastal waters, with mud to sandy mud bottoms. Monotypic.

\section{Miichthys miiuy (Basilewsky, 1855)}

Sciaena miiuy Basilewsky, 1855: 221 (Seas off Beijing [Peking], China). No types at ZIN. Otolithus fauvelii Peters, 1881: 922 (Ningpo, China). Syntypes or holotype: ZMB 11155 (1). Nibea imbricata Matsubara, 1937: 38, fig. 8 (China Sea). Holotype (unique): FAKU 3512 (apparently lost).

Distribution: Japan to East China Sea.

\section{Genus Miracorvina Trewavas, 1962}

Miracorvina Trewavas, 1962: 170. Feminine. Sciaena angolensis Norman, 1935. Type by original designation.

Southeastern Atlantic, over sand and rock bottoms in slope and shelf waters, in temperatures which are below the thermocline. Monotypic.

\section{Miracorvina angolensis (Norman, 1935)}

Sciaena angolensis Norman, 1935: 14, fig. 4 (Off St. Paul de Loanda, Angola). Syntypes: BMNH 1935.5.11.117-120 (4).

Distribution: Guinea to southern Angola.

\section{Genus Nebris Cuvier, 1830}

Nebris Cuvier in Cuvier and Valenciennes, 1830: 149. Feminine. Nebris microps Cuvier, 1830. Type by monotypy.

Cheironebris Fowler, 1944: 179. Feminine. Nebris occidentalis Vaillant, 1897. Type by original designation. 
Western Atlantic and eastern Pacific in coastal and estuarine waters over sandy and muddy bottoms; two species.

Nebris microps Cuvier, 1830

Nebris microps Cuvier in Cuvier and Valenciennes, 1830: 149, Pl. 112 (Suriname). Holotype (unique): ZMB.

Distribution: Northern South America and Brazil.

\section{Nebris occidentalis Vaillant, 1897}

Nebris occidentalis Vaillant, 1897: 124 (Suriname). Holotype (unique): ZMB.

Nebris zestus Jordan and Stark in Jordan and Evermann, 1898: 1417 (Pacific Panama). Syntypes: CAS-SU 433 (2).

Distribution: Gulf of California and southern Mexico to Peru.

Comments: Synonymy according to Castro-Aguirre (1978) and Chao et al. (2001).

\section{Genus Nibea Jordan and Thompson, 1911}

Nibea Jordan and Thompson, 1911: 244, 246 Feminine. Subgenus of Sciaena, type species Pseudotolithus mitsukurii Jordan and Snyder, 1900. Type by original designation.

Japan to northern Australia and New Guinea. Eleven species.

\section{Nibea acuta (Tang, 1937)}

Pseudosciaena acuta Tang, 1937: 62, fig. 5 (Hainan Island, Gulf of Tonkin, Kwangtung Province, China). Holotype (unique): ZMUA 13210.

Distribution: South China Sea.

Comments: Regarded as synonym of Chrysochir aureus (Richardson 1846) by Trewavas (1977: 345), and Talwar (1995: 54). Valid as Nibea acuta (Tang, 1937) according to Chen et al. (1997: 72).

\section{Nibea albiflora (Richardson, 1846)}

Corvina albiflora Richardson, 1846: 226 (Canton, China). Neotype: BMNH 1905.6.6.152 designated by Trewavas 1977: 383.

Otolithus reevesii Richardson, 1846: 224 (Canton, China). Holotype (unique): BMNH uncat. (dry).

Sciaena tenlo Basilewsky, 1855: 220, Pl. 3 (fig. 1) not Pl. 1 (fig. 3) (Gulf of Pechili; Beijing Peking, China). No types at ZIN.

Corvina fauvelii Sauvage, 1881: 105 (Swatow, China). Holotype (unique): MNHN A-2920.

Distribution: China and Japan.

\section{Nibea chui Trewavas, 1971}

Pseudosciaena albida (nec Cuvier) Bleeker, 1863a: 145 (Amoy).

Nibea chui Trewavas, 1971: 456 (Hong Kong). Syntypes: BMNH 1939.1.17.7-8 or 17-18 (2). Distribution: China and Japan.

\section{Nibea coibor (Hamilton, 1822)}

Bola coibor Hamilton, 1822: 78, 368 (Ganges River estuaries, India). No types known. Not the same as Bola coitor Hamilton in same work.

Distribution: Ganges River estuaries, Sri Lanka, Sumatra, Philippines, China, Viet Nam, and Australia.

\section{Nibea leptolepis (Ogilby, 1918)}

Sciaena leptolepis Ogilby, 1918: 87, Pl. 25 (Croker Island, Northern Territory, Australia). Holotype (unique): QM I.1534.

Distribution: Along coasts of northern Australia. 
Comments: Previously regarded as a synonym of Nibea soldado (Lacepède, 1802), it was resurrected by Sasaki (2001: 3159). Tentatively placed in genus Austronibea by Lo et al. (2017: 11).

\section{Nibea maculata (Bloch and Schneider, 1801)}

Johnius maculatus Bloch and Schneider, 1801: 75 (Tranquebar [=Tharangambadi], India). Possible syntypes: ZMB 8732 (1, dry), 8798 (1, stuffed), 8805 (1, left skin).

Distribution: Coasts of Pakistan, India and Sri Lanka, probably extending to Thailand and Malaysia.

\section{Nibea microgenys Sasaki, 1992}

Nibea microgenys Sasaki, 1992: 5, figs. 2-5 (Embley River, Weipa, northern Queensland, Australia). Holotype: QM I.26784. Paratypes: AMS I.26864-002 (1); NTM S.10177-002 (3), S.11142-001 (1), S.12162-002 (1), S.12182-001 (2); QM I.22693 (3), I.23792 (1), I.26548 (3); WAM P.13689-001 (1).

Distribution: Northern Australia and New Guinea.

\section{Nibea mitsukurii (Jordan and Snyder, 1900)}

Pseudotolithus mitsukurii Jordan and Snyder, 1900: 356, Pl. 13 (Bay of Tokyo, Japan). Holotype (unique): USNM 49407.

Distribution: Japan and the East China Sea.

\section{Nibea semifasciata Chu, Lo and Wu, 1963}

Nibea semifasciata Chu, Lo and Wu, 1963: 51 [English p. 91], figs. 28, 54, 80 (Cha-po, Kwangtung Prov., China). Holotype (unique): SFC 57-0337.

Distribution: Japan to New Guinea westward to Sri Lanka.

\section{Nibea soldado (Lacepède, 1802)}

Holocentrus soldado Lacepède, 1802: 344, 389 ("Cayenne" erroneous, should be Indo-West Pacific). Syntypes: MNHN 0000-7606 (1, dry) no locality.

Corvina miles Cuvier, 1829: 173 (Vizagapatam, India). No types known. Cuvier specimens: MNHN A-5550 (1) Puducherry, 7607 or 7606 (1, dry). Bauchot and Desoutter (1987: 9) treated MNHN A-5550 as holotype.

Sciaena miles Cuvier in Cuvier and Valenciennes, 1830: 94 Puducherry and Vizapatam, India; Java, Indonesia. Nomen novum, unneeded replacement name for Holocentrus soldado Lacepède, 1802.

Corvina wolffii Bleeker, 1851b: 66 (Bandjarmasin, Borneo, Indonesia. Holotype (unique): RMNH 5982 (1 of 20).

Corvina sampitensis Bleeker, 1852: 421 (Sampit, South Borneo, Indonesia). Holotype (unique): RMNH 5982 (1 of 20). Bleeker specimens: MNHN 0000-5982 (2).

Corvina celebica Bleeker, 1854b: 244 (Makassar, Sulawesi, Indonesia. Holotype (unique): RMNH 5982 (1 of 20).

Sciaena muelleri Steindachner, 1879b: 29 (Cleveland Bay, near Townsville, Queensland, Australia, 19¹6' S, 14648' E). Holotype (unique): SMNS 2267.

Corvina argentea Macleay, 1883: 204 (Lower Burdekin River, Queensland, Australia). Lectotype: AMS I.14578. Paralectotypes: AMS I.14579-81 (1, 1, 1), A.18241 (1), A.18243 (1), A.18245 (1).

Distribution: India, Sri Lanka, Indonesia, Hainan, Queensland, and Purari System, New Guinea north to Philippines.

\section{Nibea squamosa Sasaki, 1992}

Nibea semifasciata (nec Chu et al.) Roberts, 1978: 50, fig. 33 (Fly River, Papua New Guinea). Nibea sp. Allen, 1991: 139, fig. 22A (New Guinea). 
Nibea squamosa Sasaki, 1992: 1, figs. 1-4 (Lake Herbert Hoover, main stream of Middle Fly R., Fly R., Papua New Guinea, $7^{\circ} 14^{\prime}$ S, $141^{\circ} 08^{\prime} 18^{\prime \prime}$ E). Holotype: USNM 217221. Paratypes: AMS I.21481-001 ex USNM 217223 (1), I.22081-015 (1); NTM S.11197-001 (1), S.11400001 (2), S.11401-001 (1), S.11566-001 (3), S.12585-001 (1); QM I.11560 (1), I.20043-44 (1, 1), I.20066 (1), I.25443 (1), I.26267 (1); USNM 217222 (2).

Distribution: Northern Australia and New Guinea.

\section{Genus Odontoscion Gill, 1862}

Odontoscion Gill, 1862: 18. Masculine. Type species Corvina dentex Cuvier, 1830. Type by original designation.

Amphi-American in coral reefs and offshore waters over sandy bottoms; three species.

Comments: The genus name Larimodon Bleeker (ex Kaup), 1876: 329 often appears in synonymy with Odontoscion Gill, 1862 and then in Bleeker, 1876 as above; however it was never used as an available name, and therefore is not available and with no type validly established.

\section{Odontoscion dentex (Cuvier, 1830)}

Corvina dentex Cuvier in Cuvier and Valenciennes, 1830: 139, Pl. 109 (Dominican Republic). Holotype (unique): MNHN 0000-0144.

Distribution: Florida, Cuba, and Brazil; absent from Bahamas and most West Indies.

\section{Odontoscion eurymesops (Heller and Snodgrass, 1903)}

Corvula eurymesops Heller and Snodgrass, 1903: 195 (Santa María Island Charles Island, Galápagos Islands). Holotype: CAS-SU 6361. Paratypes: CAS-SU 8553 not 3553 (3) = 12305-07 as originally given.

Distribution: Galápagos Islands.

\section{Odontoscion xanthops Gilbert, 1898}

Odontoscion xanthops Gilbert in Jordan and Evermann, 1898: 1426 (Pacific Panama). Holotype (unique): CAS-SU 5519.

Distribution: Gulf of California to Peru.

\section{Genus Ophioscion Gill, 1863}

Ophioscion Gill, 1863b: 164. Masculine. Type species Ophioscion typicus Gill, 1863. Type by monotypy.

Sigmurus Gilbert in Jordan and Evermann, 1898: 1446. Masculine. Subgenus of Ophioscion, type species Corvina vermicularis Günther, 1867. Type by original designation.

Nine species, seven of which in the eastern Pacific and two in the western Atlantic. The identity of several species is doubtful and the genus is in need of revision.

\section{Ophioscion adustus (Agassiz, 1831)}

Corvina adusta Agassiz in Spix and Agassiz, 1831: 126, Pl. 70 (Atlantic off Brazil). Possible syntypes: MHNN 312 (1).

?Corvina gillii Steindachner, 1867: 335 (Rio de la Plata, Argentina). Holotype (unique): NMW.

Sciaena unicirrata Larrañaga, 1923: 384 (Uruguay). No types known.

Distribution: Brazil, Uruguay, Argentina.

\section{Ophioscion gomezi Cervigón, 2011}

Ophioscion gomezi Cervigón, 2011: 96, fig. 1 (La Restinga, Isla de Margarita, Venezuela). Holotype: MMM 250. Paratypes: MMM 251 (16).

Distribution: Venezuela. 


\section{Ophioscion imiceps (Jordan and Gilbert, 1882)}

Sciaena imiceps Jordan and Gilbert, 1882a: 309 (Bay of Panama). Syntypes: USNM 29432 (1), 29481 (1), 29489 (1).

Distribution: Southern Mexico to northern Ecuador.

\section{Ophioscion punctatissimus Meek and Hildebrand, 1925}

Ophioscion punctatissimus Meek and Hildebrand, 1925: 644, Pl. 68 (Cristobal, Canal Zone, Panama). Holotype: USNM 81766. Paratypes: FMNH 21670-71 (1, 1); USNM 80765-66 (1, 1).

Ophioscion panamensis Schultz, 1948: 126, 134, fig. 8 (Fox Bay, Colón, Atlantic Panama). Holotype: USNM 122612. Paratypes: USNM 81204-07 (3, 4, 1, 1), 128260 (1).

Distribution: Central western Atlantic: Puerto Rico and from Panama to Brazil.

\section{Ophioscion scierus (Jordan and Gilbert, 1884)}

Sciaena sciera Jordan and Gilbert, 1884: 480 (Mazatlán, Sinaloa, Mexico; or Panama). Syntypes: USNM 29499 (?).

Ophioscion obscurus Hildebrand, 1946: 294, fig. 63 (Lobos de Tierra Bay, Peru). Holotype: USNM 128029. Paratypes: USNM 128030 (1).

Distribution: Gulf of California to Peru.

Comments: I follow the opinion of Chao (1995) in regarding O. obscurus as a junior synonym of $O$. scierus.

\section{Ophioscion simulus Gilbert, 1898}

Ophioscion simulus Gilbert in Jordan and Evermann, 1898: 1449 (Pacific Panama). Holotype (unique): CAS-SU 5516.

Distribution: Costa Rica and Panama.

\section{Ophioscion strabo Gilbert, 1897}

Ophioscion strabo Gilbert, 1897: 444, Pl. 50 (San Juan Lagoon, south of Guaymas, western coast of Mexico). Holotype: USNM 47742. Paratypes: (5) CAS-SU 436 (4).

Corvina miacanthus Boulenger, 1899: 5 (Port of Guayaquil, Ecuador). Syntypes: BMNH 1898.12.31.13 (1), MZUT 1574 (2).

Distribution: Gulf of California to Peru.

Comments: Corvina miacanthus Boulenger, 1899 represents a new synonym of Ophioscion strabo Gilbert, 1897; the two syntypes at the Regio Museo di Torino (Tortonese, 1940) have been examined. Not listed in the checklists of the fishes of Ecuador (Cobo and Massay, 1969; Béarez, 1996; Jiménez-Prado and Béarez, 2004), but present in Ecuador.

\section{Ophioscion typicus Gill, 1863}

Ophioscion typicus Gill, 1863b: 165 (Pacific Panama). Holotype (unique): USNM $5163=$ 22861.

Corvina ophioscion Günther, 1868: 428 (Panama). Syntypes:probably BMNH 1866.1.19.18 (1).

Distribution: Costa Rica to Ecuador.

Ophioscion vermicularis (Günther, 1867)

Corvina vermicularis Günther, 1867: 601 (Pacific Panama). Holotype (unique): BMNH 1865.7.20.13. Described in more detail in Günther 1868: 427, Pl. 67 (fig. 2).

Distribution: Panama to northern Peru.

\section{Genus Otolithes Oken, 1817}

Otolithes Oken (ex Cuvier), 1817: 1182. Masculine. Type species Johnius ruber Bloch and Schneider, 1801. Type by subsequent designation by Gill 1861c: 80 .

Otolithus by Cuvier 1829: 172 (unjustified emendation). 
Indian Ocean; three species.

Otolithes arabicus Lin, Qurban, Shen and Chao, 2019

Otolithes arabicus Lin, Qurban, Shen and Chao, 2019: 14, figs. 6-7 (Jubail Fish Market,

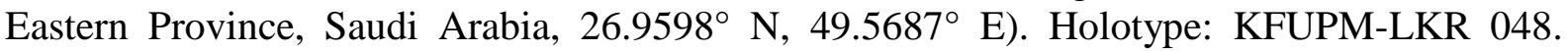
Paratypes: KFUPM-LKR-047, KFUPM-LKR-113 to KFUPM-149 (47), NNMB-P 29769.

Distribution: Western Persian Gulf to the Gulf of Oman, northwestern Indian Ocean.

\section{Otolithes cuvieri Trewavas, 1974}

Otolithus ruber Cuvier in Cuvier and Valenciennes (nec Bloch and Schneider), 1830: 60. (Puducherry, India).

Otolithes cuvieri Trewavas, 1974: unnumbered (Malabar, India). Syntypes: BMNH 1898.11.18.29 (1) "paratype"; MNHN 0000-7617 (1) "holotype" and MNHN 1987-0159 ex MNHN 0000-7617 (1) "paratype".

Distribution: North Indian Ocean.

Comments: This nominal species was proposed as a new name for Otolithus ruber (not Bloch and Schneider, 1801) Cuvier, 1830, with 2 literature sources provided; and Trewavas' name was not technically a replacement name. This species was later illustrated and described in more detail in Trewavas (1977: 351, Pl. 2). Type specimens considered syntypes with no holotype established in the 1974 description, but a holotype and two paratypes are listed in Trewavas (1977). Valid (Sasaki, 1996: 88).

\section{Otolithes ruber (Bloch and Schneider, 1801)}

Johnius ruber Bloch and Schneider, 1801: 75, Pl. 17 (Indian Ocean). Holotype (unique): ZMB 5528 (right skin).

Otolithus versicolor Cuvier, 1829: 173 (Vishakhapatnam [Vizagapatam], India). No types known.

Otolithus argenteus Cuvier (ex Kuhl and Van Hasselt) in Cuvier and Valenciennes, 1830: 62 (Batavia [=Jakarta], Indonesia). Syntypes: MNHN 0000-7510 (1) Malabar, 0000-7513 (1) Malabar.

Otolithus tridentifer Richardson, 1846: 225 (Canton, China). Holotype (unique) (?): BMNH 2004.11.24.3 (left half skin).

Otolithus orientalis Seale, 1910: 281, Pl. 4 (Sandakan, Sabah state, Borneo, East Malaysia). Holotype (unique): BSMP 2744.

Distribution: South and East Africa, Madagascar and Persian Gulf east to Philippines, north to northern Vietnam and southern China, south to Queensland (Australia).

\section{Genus Otolithoides Fowler, 1933}

Sciaenoides Blyth, 1860: 139. Masculine. Type species Otolithus biauritus Cantor, 1849. Type by subsequent designation. Type designated by Bleeker 1876: 330 .

Otolithoides Fowler, 1933: 364. Masculine. Type species Otolithus biauritus Cantor, 1849. Type by original designation.

Pama Fowler, 1933: 360. Feminine. Type species Bola pama Hamilton, 1822. Type by original designation.

Indo-West Pacific in coastal and inshore waters; two species.

Comments: Sciaenoides Richardson, 1843: 8 is an available name; therefore, Otolithoides is an unneeded replacement name. Sciaenoides Blyth, 1860 has been regarded as valid by Kottelat (2013: 360) but it has never been used in current literature.

\section{Otolithoides biauritus (Cantor, 1849)}

Otolithus biauritus Cantor, 1849: 1039 (Chusan. Orig. localities: Sea of Pinang [Penang], Malayan Peninsula, Singapore, Lancavy, Tenasserim Provinces). Lectotype: BMNH 
1860.3.19.171 (skin). Paralectotypes: BMNH 1860.3.19.172-3 (2, skins) Chusan. Lectotype selected by Trewavas 1977: 297.

Otolithus brunneus Day, 1873: 524 (Bombay, India). Lectotype: ZSI F906. Paralectotypes and/or Day specimens: AMS B.8193-94 (1, 1, both paralectotypes), MNHN 0000-1004 (1), RMNH 670 (1), ZSI F1024-26 (3).

Distribution: Pakistan, India, Sri Lanka to Sumatra, Borneo, and Vietnam.

\section{Otolithoides pama (Hamilton-Buchanan, 1822)}

Bola pama Hamilton-Buchanan, 1822: 79, 368, Pl. 32 (fig. 26) (Ganges tidal estuaries, Calcutta, India). No types known.

Sciaenoides hardwickii Blyth, 1860: 139 (mouth of the Ganges River). Holotype: possibly ZSI 884.

Distribution: India.

\section{Genus Pachypops Gill, 1861}

Pachypops Gill, 1861c: 87. Masculine. Type species Micropogon trifilis Müller and Troschel, 1849. Type by original designation.

Freshwaters of South America; genus revised by Casatti (2002) with three species.

\section{Pachypops fourcroi (Lacepède, 1802)}

Perca fourcroi Lacepède, 1802: 398, 424 (no locality). Holotype (unique): MNHN 00007539.

Corvina biloba Cuvier in Cuvier and Valenciennes, 1830: 112 (no locality). Holotype (unique): MNHN 0000-7683.

Corvina furcraea Cuvier in Cuvier and Valenciennes, 1830: 111 (misspelling).

Pachyurus nattereri Steindachner, 1863: 171, Pl. 3 (Rio Branco or Rio Negro, Brazil). Lectotype: NMW 15178: 1. Paralectotypes: NMW 15178: 2-3 (2). Spelled natteri on p. 171, as nattereri on p. 175 and on Pl. 3; nattereri regarded as intended. Lectotype selected by Casatti (2002a: 5).

Distribution: Amazon and Orinoco River basin, and rivers of the Guianas.

Comments: Taxonomic decision of Bauchot and Desoutter (1987) and Casatti (2002a).

Pachypops pigmaeus Casatti, 2002

Pachypops pigmaeus Casatti 2002a: 14, fig. 7 (Jamarizinho, Rio Machado, Rondônia, Brazil). Holotype: MZUSP 34108. Paratypes: MZUSP 34105 (7), 34113-14 (1, 1).

Distribution: Amazon River basin in Branco and Madeira River drainages, Brazil.

\section{Pachypops trifilis (Müller and Troschel, 1848)}

Micropogon trifilis Müller and Troschel, 1848: 622 (Guyana). Holotype: ZMB 936.

Pachypops camposi Fowler, 1954: 252, fig. 843 (Rupununi River, Guyana). Holotype (unique): ANSP 39773.

Distribution: Amazon River basin and rivers of the Guianas.

Comments: Taxonomic decision of Chao (pers. comm. 1982 in Böhlke, 1984).

\section{Genus Pachyurus Agassiz, 1831}

Lepipterus Cuvier in Cuvier and Valenciennes, 1830: 151 Masculine. Type species Lepipterus francisci Cuvier, 1830. Type by monotypy.

Pachyurus Agassiz in Spix and Agassiz, 1831: 125, 127. Masculine. Type species Pachyurus squamipennis Agassiz, 1831.

Freshwaters of South America; genus revised by Casatti (2001) with 10 species.

Comments: Lepipterus Cuvier is not preoccupied by Lepipterus Rafinesque, 1815 in fishes (nomen nudum). Casatti (2001: 135) recognizes Pachyurus over the older name Lepipterus on the basis of Art. 23.9.1.1. of the ICZN. 


\section{Pachyurus adspersus (Steindachner, 1879)}

Pachyurus (Lepipterus) adspersus Steindachner, 1879a: 123 (Rio Santo Antonio, Estado de Minas Gerais, Brazil). Lectotype: NMW 31450. Paralectotypes: NMW 15179 (2). Lectotype selected by Casatti (2001: 150).

Distribution: Atlantic Versant Rivers of Brazil, including Mucuri, Doce, and Paraíba do Sul River basins.

\section{Pachyurus bonariensis Steindachner, 1879}

Pachyurus (Lepipterus) bonariensis Steindachner, 1879a: 126 (Río de la Plata, Argentina). Lectotype: NMW 15181. Paralectotypes: should be only 3, originally 4 syntypes NMW 15193 (1), 78598 (2), 84794 (1). Lectotype selected by Casatti (2001: 154).

Pachyurus paranensis Daneri, 1956: 6 (Río Paraná, frente Est. Hidrobiol. Rosario, Argentina). Holotype: MACN 4234. Paratypes: MACN 473 (3), 489 (4), 4050 (10), 4234 (9).

Distribution: Amazonas, Rio Grande do Sul, Rio Uruguay, Rio Paraguay.

\section{Pachyurus calhamazon Casatti, 2001}

Pachyurus calhamazon Casatti, 2001: 167, fig. 8C (Rio Branco, Estado de Roraima, Brazil). Holotype (unique): MZUSP 66684.

Distribution: Branco River, Brazil.

\section{Pachyurus francisci (Cuvier, 1830)}

Lepipterus francisci Cuvier in Cuvier and Valenciennes, 1830: 152, Pl. 113. (Rio São Francisco, Brazil). Holotype (unique): MNHN A-5600 (dry).

Lepipterus corvina Reinhardt, 1851: 30 (Rio das Velhas, Brazil). Lectotype: ZMUC 5. Paralectotypes: (1) ZMUC 3 (1) or 4 (1). Lectotype selected by Casatti 2001: 140.

Distribution: Rio São Francisco, Rio Parana.

\section{Pachyurus gabrielensis Casatti, 2001}

Pachyurus gabrielensis Casatti, 2001: 163, fig. 8B (Cachoeira São Gabriel, Rio Negro, Estado do Amazonas, Brazil). Holotype: MZUSP 34093. Paratypes: MZUSP 49721 (8, 1 $\mathrm{d} \& \mathrm{c})$.

Distribution: Amazon and Orinoco River basins: Brazil, Colombia, Peru and Venezuela.

\section{Pachyurus junki Soares and Casatti, 2000}

Pachyurus junki Soares and Casatti, 2000: 500, fig. 1 (Rio Solimões, Coari, Estado do Amazonas, Brazil). Holotype: INPA 619. Paratypes: CAS 32058 (1), INPA 4049 (5), MCP 15121 (2), MZUSP 37572 (1).

Distribution: Amazon River basin, Brazil and Colombia.

\section{Pachyurus paucirastrus Aguilera, 1983}

Pachyurus paucirastrus Aguilera, 1983a: 121, fig. 1 (Rio Tocantins, Capuerana, Brazil). Holotype: MNRJ 11176. Paratypes: MBUCV-V-13869 (1), V-14130 (4).

Distribution: Amazonia: Tocantins River basin.

\section{Pachyurus schomburgkii Günther, 1860}

Pachyurus schomburgkii Günther, 1860: 282 (Rio Capin = Capim, Pará State, and Caripe Pará, Brazil). Syntypes: (2) BMNH 1849.11.8.22 (1) Rio Capin = Capim.

Distribution: Amazon and Orinoco River basins: Bolivia, Brazil, Colombia, Peru and Venezuela.

\section{Pachyurus squamipennis Agassiz, 1831}

Pachyurus squamipennis Agassiz in Spix and Agassiz, 1831: 128, Pl. 71 (São Francisco River basin, Brazil). No types known.

Pachyurus lundii Reinhardt, 1875: 111 (Rio das Velhas, Brazil). Lectotype: ZMUC 2. Paralectotypes: ZMUC 1 (1). Lectotype selected by Casatti 2001: 142. 
Distribution: Rio Sao Francisco.

\section{Pachyurus stewarti Casatti and Chao, 2002}

Pachyurus stewarti Casatti and Chao, 2002: 2, fig. 1 (Río Aguarico, in the confluence with Río Pushino, 0².6' N, 7654.4' W, Sucumbíos, Ecuador). Holotype: FMNH 101957. Paratypes: FMNH 94410 (2), 94116 (1), 101956 (1).

Distribution: Napo River drainage, Ecuador.

\section{Genus Panna Lal Mohan, 1969}

Panna Lal Mohan, 1969: 296. Feminine. Type species Otolithus microdon Bleeker, 1849. Type by original designation.

Indo-West Pacific in coastal waters, also in river mouths and estuaries. The genus has been revised by Sasaki 1995 recognizing three species.

\section{Panna heterolepis Trewavas, 1977}

Panna heterolepis Trewavas, 1977: 308, fig. 6 (Calcutta, India). Holotype (unique): BMNH 1889.2.1.3137.

Distribution: Sri Lanka, India, Bangladesh and Burma.

\section{Panna microdon (Bleeker, 1849)}

Otolithus microdon Bleeker, 1849a: 10 (Jakarta [Batavia]; Samarang [Semarang]; Surabaya, Java, Indonesia). Syntypes and/or Bleeker specimens: (at least 4) ?BMNH 1880.4.21.134-135 (2), RMNH 5978 (some of 6), SMNS 10630 (1).

Distribution: Western Indonesia, Malaysia, southern Vietnam, India, and Burma.

\section{Panna perarmatus (Chabanaud, 1926)}

Sciaenoides perarmatus Chabanaud, 1926: 266 (Gulf of Thailand; Indo-China). Syntypes: MNHN 0000-8998 (1), A-4214 (4), A-4215 (1), MNHN Lab. Pêches Coloniales now Pêches Outre-mer (1).

Distribution: Gulf of Thailand and western Borneo.

\section{Genus Paralonchurus Bocourt, 1869}

Paralonchurus Bocourt, 1869: 21. Masculine. Type species Paralonchurus petersii Bocourt, 1869. Type by monotypy.

Polycirrhus Bocourt, 1869: 23. Masculine. Type species Polycirrhus dumerilii Bocourt, 1869. Type by monotypy.

Polyclemus Berg, 1895: 54. Masculine. Type species Polycirrhus dumerilii Bocourt, 1869. Type by being a replacement name. Unneeded replacement for Polycirrhus Bocourt, 1869, thought to be preoccupied by Polycirrus Grube, 1850 in Polycirridae, Polychaeta, Terebellida. Zonoscion Jordan and Evermann, 1896: 401. Masculine. Subgenus of Paralonchurus, type species Polycirrhus rathbuni Jordan and Bollman, 1890. Type by original designation.

Zaclemus Gilbert in Jordan and Evermann, 1898: 1480. Masculine. Subgenus of Paralonchurus, type species Paralonchurus goodei Gilbert, 1898. Type by original designation.

Six species according to Chao (1978); seven species included by Sasaki (1989) and they are listed here.

\section{Paralonchurus brasiliensis (Steindachner, 1875)}

Genyanemus brasiliensis Steindachner, 1875a: 476 (Para and Santos, Brazil). Syntypes: (several) BMNH 1879.5.14.289 (1) R. Plata; MCZ 8626 (1) Rio, MCZ 8627 (1) Santos, NMW 60468 (2).

Micropogon ornatus Günther, 1880: 13, Pl. 7 (fig. A) (Rio de la Plata mouth, Challenger station 321, depth 13 fathoms). Holotype (unique): BMNH 1879.5.14.289. 
Distribution: Brazil to Argentina.

\section{Paralonchurus dumerilii (Bocourt, 1869)}

Polycirrhus dumerilii Bocourt, 1869: 23 (Union, El Salvador). Holotype (unique): MNHN A1001.

Genyonemus fasciatus Steindachner, 1875a: 473 (Panama Bay). Syntypes: (several) NMW 137 (2), 77762 (1).

Distribution: El Salvador to northern Chile.

\section{Paralonchurus elegans Boeseman, 1948}

Paralonchurus elegans Boeseman, 1948: 363, figs. 1a-b (Bokelbere Creek, Coronie sea coast, Suriname). Holotype (unique): RMNH 390.

Distribution: Northern South America.

\section{Paralonchurus goodei Gilbert in Jordan and Evermann, 1898}

Paralonchurus (Zaclemus) goodei Gilbert in Jordan and Evermann, 1898: 1480 (Pacific Panama). Lectotype: CAS-SU 5517. Paralectotypes: BMNH 1903.5.15.100 (1), CAS-SU 6814 (4), USNM 50350 (1). Appeared first as nomen nudum in Jordan and Evermann 1896: 401. As Polyclemus goodei in Gilbert in Gilbert and Starks, 1904: 135, Pl. 20 (figs. 40-40a), with lectotype established by use of "type specimen" in caption to figure 40.

Distribution: Gulf of California to Peru.

\section{Paralonchurus peruanus (Steindachner, 1875)}

Genyanemus peruanus Steindachner, 1875a: 471 (Paita and Callao, Peru). Syntypes: (several) NMW 15182 (4), 58138 (2), 78571 (2), 84795 (1); USNM 153619 [ex MCZ 8625] (2), 53620 [ex MCZ 8620] (2).

Micropogon fasciatus de Buen, 1961: 20 (Arica, Chile). Holotype (unique): EBMC 10374 (lost).

Distribution: Panama Bay to northern Chile.

Comments: Placement of M. fasciatus in the genus Paralonchurus follows the opinion of Kong and Valdés (1990: 50).

\section{Paralonchurus petersii Bocourt, 1869}

Paralonchurus petersii Bocourt, 1869: 22 (Union, El Salvador). Holotype (unique): MNHN A-0979.

Distribution: El Salvador to Ecuador.

Comments: A synonym of $P$. dumerilii according to Bauchot and Desoutter (1987); valid according to many authors (e.g. Allen and Robertson, 1994: 166; Bussing and López, 1994: 126; Chao, 1995: 1493).

\section{Paralonchurus rathbuni (Jordan and Bollman, 1890)}

Polycirrhus (Zonoscion) rathbuni Jordan and Bollman, 1890: 162 (Pacific Panama). Syntypes: CAS-SU 408 (1), USNM 41170 (2).

Distribution: Panama to Peru.

\section{Genus Paranebris Chao, Béarez and Robertson, 2001}

Paranebris Chao, Béarez and Robertson, 2001: 82. Feminine. Type species Paranebris bauchotae Chao, Béarez and Robertson, 2001 by original designation.

Eastern Pacific on muddy and sandy bottoms; monotypic.

\section{Paranebris bauchotae Chao, Béarez and Robertson, 2001}

Paranebris bauchotae Chao, Béarez and Robertson 2001: 82, figs. 1-3, 4a (Golfo de San Miguel, Panama, $8^{\circ} 18^{\prime} \mathrm{N}, 78^{\circ} 28^{\prime} \mathrm{W}$, depth 12 meters). Holotype: USNM 360918. Paratypes: MCZ 157272 (1), MNHN 1988-261 (1). 
Distribution: Gulf of Panama.

Genus Paranibea Trewavas, 1977

Paranibea Trewavas, 1977: 370. Feminine. Type species Corvina semiluctuosa Cuvier, 1830. Type by original designation.

Indo-west Pacific, in coastal waters; monotypic.

\section{Paranibea semiluctuosa (Cuvier, 1830)}

Corvina semiluctuosa Cuvier in Cuvier and Valenciennes, 1830: 97, Pl. 106 (Malabar, India). Lectotype: MNHN 0000-7537. Paralectotypes: MNHN 1987-0155 ex MNHN 0000-7537 (1), 0000-7592 (1) Goa, 7596 (2) Puducherry; ?SMF 510 ex MNHN in 1830 (1) Puducherry; ?ZMB 876 ex MNHN (1) Puducherry. Type catalog: Bauchot and Desoutter 1987: 9-10. Lectotype selected by Trewavas 1977: 370.

Distribution: Pakistan to western Indonesia.

\section{Genus Pareques Gill, 1876}

Pareques Gill in Goode, 1876: 50. Masculine. Type species Grammistes acuminatus Bloch and Schneider, 1801. Type by monotypy.

Eastern Pacific (four species) and western Atlantic (three species). Found on sandy and muddy bottoms of high salinity and coral reefs (Chao, 1978).

\section{Pareques acuminatus (Bloch and Schneider, 1801)}

Grammistes acuminatus Bloch and Schneider, 1801: 184 (no locality stated). No types known.

Eques lineatus Cuvier in Cuvier and Valenciennes, 1830: 169 (Brazil). Syntypes: MNHN 0043 (1), 7475 (1).

Eques pulcher Steindachner, 1867: 349 (Barbados, West Indies). Syntypes: NMW 75797 (3).

Distribution: Bermuda, Florida, and Bahamas to Brazil.

Pareques fuscovittatus (Kendall and Radcliffe, 1912)

Eques fuscovittatus Kendall and Radcliffe, 1912: 125, Pl. 5 (fig. 1) (Acapulco, Pacific coast of Mexico). Holotype (unique): USNM 65494.

Distribution: Gulf of California to Puerto Vallarta (Mexico).

\section{Pareques iwamotoi Miller and Woods, 1988}

Pareques iwamotoi Miller and Woods, 1988: 89, fig. 1 (South of Pensacola, Oregon sta. 698, 3003'36" N, 8655'36" W, Florida, U.S.A., $101 \mathrm{~m}$ ). Holotype: USNM 174972. Paratypes: FMNH 45531-35 (1, 2, 1, 1, 1), 64166-67 (4, 1), 65407 (1); UF 207133 ex UMML 7133 (1). Additional material: CAS (6 lots, 12); FMNH (4 lots, 9); UF ex UMML (25 lots, 93).

Distribution: Western Atlantic.

Comments: Status as determined by Boschung (1992).

Pareques lanfeari (Barton, 1947)

Eques lanfeari Barton, 1947: 1, fig. 1 (Off Talara, Piura Province, Peru, depth 250 feet). Holotype: AMNH 17081.

Distribution: Ecuador and Peru.

\section{Pareques perissa (Heller and Snodgrass, 1903)}

Sciaena perissa Heller and Snodgrass, 1903: 197 (Tagus Cove, Albermarle I., Galápagos Islands). Holotype: CAS-SU 6360. Paratypes: CAS-SU 8552 (4).

Distribution: Galápagos Islands.

Pareques umbrosus (Jordan and Eigenmann, 1889) 
Eques acuminatus umbrosus Jordan and Evermann, 1889: 440 (Charleston, South Carolina, U.S.A.). Holotype (unique): USNM 25981 or CAS 18958.

Distribution: Western Atlantic from Chesapeake Bay to Florida.

\section{Pareques viola (Gilbert, 1898)}

Eques viola Gilbert in Jordan and Evermann, 1898: 1486 (Bay of Panama, Pacific Panama). Lectotype: CAS-SU 5521. Paralectotypes: (2) and Gilbert specimens: BMNH 1903.5.15.157158 (2), CAS-SU 5909 (3), USNM 50420 (1), ZMB 15991 ex CAS-SU (1).

Distribution: Panama to Peru.

\section{Genus Pennahia Fowler, 1926}

Pennahia Fowler, 1926: 776. Feminine. Subgenus of Johnius, type species Otolithus macrophthalmus Bleeker 1849. Type designated by ICZN; on Official List (Opinion 1237). Indo-West Pacific in shallow coastal mud bunks; five species.

\section{Pennahia anea (Bloch, 1793)}

Johnius aneus Bloch, 1793: 135, Pl. 357 (Malabar, India). Syntypes: ZMB 8726 (1, dry, lost). Labrus cupreus Shaw, 1803: 527 (Indian Seas). Nomen novum, unneeded replacement name for Johnius aneus Bloch 1793.

Otolithus macrophthalmus Bleeker, 1849b: 16 (Jakarta, Java; Bantam; Semarang, Java; Pasuruan, Java, Indonesia). Syntypes: SMNS 10629 (1).

Johnius resplendens Hombron and Jacquinot in Jacquinot and Guichenot, 1853: 45, Pl. 5 (fig. 1) (no locality). Based on a picture of a fish captured by the Astrolabe or La Zélée; type apparently not available.

Otolithus leuciscus Günther, 1872: 398 (Manila Bay, Philippines). Syntypes: BMNH 1872.10.18.128-129 (2).

Distribution: Persian Gulf to Taiwan and south through the Philippines and Borneo to about the southern tip of Java.

Comments: Otolithus macrophthalmus Bleeker, 1850 is a junior synonym as recently established by Sasaki (1994)

\section{Pennahia argentata (Houttuyn 1782)}

Sparus argentatus Houttuyn, 1782: 319 (Nagasaki, Japan). Neotype: RMNH 26848 ex RMNH 5973.

Corvina argentata Cuvier in Cuvier and Valenciennes 1830: 114. An unjustified emendation of Sparus argenteus Houttuyn 1782.

Pseudosciaena schlegeli Bleeker, 1879: 9 (Nagasaki, Japan). Lectotype: RMNH 26848 ex RMNH 5973. Paralectotypes: RMNH 5973 (2). Lectotype designation not researched.

Sciaena iharae Jordan and Metz, 1913: 37, Pl. 7 (fig. 2) (Pusan, South Korea). Holotype (unique): FMNH 55629 ex CM 4371.

Distribution: South China Sea, East China Sea, Yellow Sea, Bohai Sea.

\section{Pennahia ovata Sasaki, 1996}

Pennahia ovata Sasaki, 1996b: 11, figs. 1-3 (Myanmar, 21 ${ }^{\circ} 00^{\prime}$ N, 90 $59^{\circ}$ E, depth 23-25 meters). Holotype: USNM 324181. Paratypes: USNM 324492 (1), 324525 (3), 324585 (50), 325068 (1), 325283 (1), 325472 (3).

Distribution: Bay of Bengal.

\section{Pennahia macrocephala (Tang, 1937)}

Pseudosciaena macrocephalus Tang, 1937: 70, Pl. 1 (fig. 2) (Amoy, China). Holotype (unique): ZMUA 20831.

Distribution: Taiwan to southern China, off Sarawak, eastern tip of the Malay Peninsula and southern Java. 


\section{Pennahia pawak (Lin, 1940)}

Argyrosomus pawak Lin, 1940: 248, fig. 3 (Hong Kong). Holotype: not researched. Paratypes: (3).

Distribution: Taiwan to southern China, Gulf of Thailand, and southwest Java.

\section{Genus Pentheroscion Trewavas, 1962}

Pentheroscion Trewavas, 1962: 170. Masculine. Type species Sciaena mbizi Poll, 1950. Type by original designation.

Eastern Atlantic, in deep shelf and upper slopes over mud, sand and rock bottoms; monotypic.

\section{Pentheroscion mbizi (Poll, 1950)}

Sciaena mbizi Poll, 1950: 8, fig. 2 (Atlantic, 37 mile west-southwest of Landana, $5^{\circ} 25^{\prime} \mathrm{S}$,

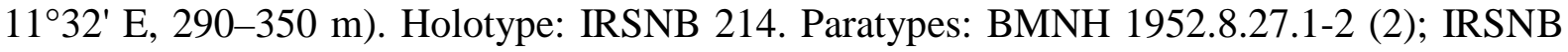
215-229 (1, 6, 5, 1, 3, 2, 2, 3, 2, 4, 3, 1, 3, 1, 1); MRAC 97691-709 (19).

Distribution: Guinea to southern Angola.

\section{Genus Petilipinnis Casatti, 2002}

Petilipinnis Casatti, 2002b: 171. Feminine. Type species Corvina grunniens Jardine and Schomburgk, 1843.

South America, in freshwater; monotypic.

\section{Petilipinnis grunniens (Jardine and Schomburgk, 1843)}

Corvina grunniens Jardine and Schomburgk in Schomburgk, 1843: 136, Pl. 2 (Commaca Island, Essequibo River, Guyana). No types known.

Distribution: Essequibo River, British Guiana.

\section{Genus Plagioscion Gill, 1861}

Plagioscion Gill, 1861c: 82. Masculine. Type species Type species Sciaena squamosissima Heckel, 1840 by subsequent designation by Jordan and Eigenmann, 1889: 80).

Diplolepis Steindachner, 1863: 164. Feminine. Type species Sciaena squamosissima Heckel, 1840 by original designation, name preoccupied in Hymenoptera by Diplolepis Geoffroy, 1762).

Endemic to freshwaters of South America, originally distributed in the Río Magdalena, Río Amazonas, Río Orinoco, lower Río Paraná basins and rivers of the Guianas; introduced into the upper Río Paraná and Río São Francisco basins and in artificial reservoirs of northeastern Brazil. Genus revised by Casatti 2005 recognizing five species.

\section{Plagioscion auratus (Castelnau, 1855)}

Johnius auratus Castelnau, 1855: 12, Pl. 4 (fig. 2) (Río Ucayali, Amazon basin, Peru). Holotype (unique): MNHN 0000-7622.

Corvina monacantha Cope, 1867: 402 (Near Paramaribo, Suriname). Holotype (unique): ANSP 11519.

Plagioscion auratus iquitensis Nakashima, 1941: 67 (Iquitos, Peru). Types: ?MHNJP = MUSM.

Distribution: Amazonia, Peru, Bolivia, Brazil (Rio Poti, Rio São Francisco), Venezuela, Suriname, and Guyana.

Comments: Corvina monacantha Cope is a valid Plagioscion according to Chao (1978: 44), synonym according to Casatti in Reis et al. (2003: 601).

\section{Plagioscion magdalenae (Steindachner, 1878)}

Sciaena magdalenae Steindachner, 1878b: 22, pl. 1, fig. 1 (Río Magdalena, Colombia). Holotype: material not located at NMW. Species later illustrated and described as "Sciaena 
Magdalenae n. sp. (an Sc. surinamensis Blkr. adult?)" in more detail in Steindachner 1879: 22, Pl. 1 (fig. 3).

Distribution: Colombia and Brazil.

\section{Plagioscion montei Soares and Casatti, 2000}

Plagioscion montei Soares and Casatti, 2000: 504, fig. 5 (Rio Solimões, Lago Janauacá, AM, Brazil). Holotype: INPA 15959. Paratypes: INPA 604 (18); MZUSP 34086 (1), 34090 (3).

Distribution: Amazon River basin: Brazil and Peru. Habitat: freshwater.

\section{Plagioscion squamosissimus (Heckel, 1840)}

? Sciaena flexilinea Walbaum, 1792: 320 (Suriname). No types known.

Sciaena squamosissima Heckel, 1840: 438, pl. 30 (figs. 26-28) (Río Negro and Río Branco, Brazil). Syntypes: NMW 75985 (4), 83543 (1), 92124 (1); SMF 2961 ex NMW in 1844 (1, dry). Soares and Casatti 2000: 512 treat NMW 92124 as the holotype.

Johnius crouvina Castelnau, 1855: 11, Pl. 5 (fig. 1) (Río Araguaia and Río Crixas, Brazil). Syntypes: MNHN 0000-7503 (1). The specimen MNHN 7503 has been regarded as holotype in Paris records, in Chao 1978: 44 and in Casatti 2005: 43 but original description lists several specimens, so there are potential syntypes.

Johnius amazonicus Castelnau 1855: 12, Pl. 4 (fig. 1) (Río Amazonas, Brazil). Syntypes: MNHN 0000-7504 (1), B-2772 ex MNHN 0000-7504 (1).

Pseudosciaena surinamensis Bleeker, 1873: 458, pl. 21, side figure (Suriname). Holotype (unique): RMNH 5995.

Plagioscion francisci Steindachner, 1917: 669, Pl. 2 (fig. 2) (Barra, at confluence of Rio Grande and Rio São Francisco, Brazil). Syntypes: (3) NMW 57217 (1).

Plagioscion squamosissimus iquitensis Nakashima, 1941: 68 (Iquitos, Peru). Types: ?MHNJP = MUSM.

Plagioscion casattii Aguilera and Rodrigues de Aguilera, 2000: 61, fig. 1a-c (Río Orinoco, Venezuela). Holotype: MBUCV-V-29490. Paratypes: MBUCV-V-29491 (11), MHNLS 14601 (2).

Distribution: Amazon, Orinoco, Paraná, Paraguay and São Francisco basins and rivers of the Guianas: Argentina, Bolivia, Brazil, Colombia, Ecuador, French Guiana, Guyana, Paru, Suriname and Venezuela.

\section{Plagioscion ternetzi Boulenger, 1895}

Plagioscion ternetzi Boulenger, 1895: 523, fig. 3 (Río Grande, Paraguay). Lectotype: BMNH 1895.5.17.1. Paralectotypes: BMNH 1895.5.17.2 (1). Lectotype selected by Casatti (2005: 56).

Plagioscion macdonaghi Daneri, 1954: 181, fig. 1 (Río de La Plata, Argentina). Holotype: MACN 4197. Paratypes: MACN 1994 (1), 4198 (6), 4230 (1).

Distribution: Rio Grande do Sul, Rio Paraguay (Brazil), Rio de la Plata (Uruguay), and Rio Parana medio (Argentina).

Comments: López et al. (2016: 53) listed both P. ternetzi and P. macdonaghi as valid from the Rio de la Plata.

\section{Genus Pogonias Lacepède, 1801}

Pogonias Lacepède, 1801: 137. Masculine. Type species Pogonias fasciatus Lacepède, 1801. Type by monotypy.

Pogonathus Lacepède (ex Commerson), 1803: 120. Masculine. Type species Pogonathus courbina Lacepède, 1803. Type by subsequent designation. Type apparently designated first by Jordan (1917: 65).

Pogonognathus Agassiz, 1846: 301. Unjustified emendation of Pogonathus Lacepède. 
Western Atlantic in areas with large river runoffs over sandy bottoms; two species (Azpelicueta, 2019).

\section{Pogonias cromis (Linnaeus, 1766)}

Labrus cromis Linnaeus, 1766: 479 (Carolina South Carolina, U.S.A.; Brazil; Jamaica). No types known. Based on multiple sources, including the Cromis subargenteus oblongus of Browne, 1756: 449 from Jamaica, Coracinus brasiliensis guatucupa of Ray, 1713: 96 from Brazil, 'Guatucupa' of Marggraf von Liebstadt, 1648: 177 from Brazil, and 'Drum' in manuscript description by A. Garden, South Carolina.

Pogonias fasciatus Lacepède (ex Bosc d'Antic), 1801: 137, 138 (Carolina, U.S.A.). Holotype (unique): MNHN 0000-7461. Description based on the holotype which was donated via the Netherlands to MNHN, and on Chatodon percatus in a manuscript by Louis-Augustin Bosc d'Antic (1759-1828, French diplomat and naturalist). Figured on Pl. 16 (fig. 2) of Lacepède 1800 , but with vernacular name "Pogonias Fascé".

Mugil grunniens Mitchill, 1814: 15 (New York, U.S.A.). No types known. Also in Mitchill, 1815: 405 as Labrus grunniens.

Mugil gigas Mitchill, 1814: 16 (New York, U.S.A.). No types known. Also in Mitchill, 1815: 412, Pl. 5 (fig. 10) as Sciaena gigas.

Sciaena fusca Mitchill, 1815: 409 (New York, U.S.A). No types known.

Distribution: Nova Scotia, northern Mexico and Florida.

Pogonias courbina (Lacepède, 1803)

Pogonathus courbina Lacepède, 1803: 120, 121 (Rio de la Plata). Neotype: UNMDP 4874. Original description based on Commerson manuscript. Neotype designated by Azpelicueta et al. (2019: 14).

Sciaena barbata Larrañaga, 1923: 380, 384 (Uruguay). No types known.

Distribution: Southwestern Atlantic Ocean: from the State of Rio de Janeiro (Brazil) to the south of Golfo San Matías, in Argentina.

\section{Genus Protonibea Trewavas, 1971}

Protonibea Trewavas, 1971: 458. Feminine. Type species Lutjanus diacanthus Lacepède, 1802. Type by monotypy.

Indo-West Pacific in coastal waters over muddy bottoms, off the sea-bed and ascending tidal rivers and estuaries; monotypic.

Comments: The opinion that Protonibea is a junior synonym of Megalonibea has been suggested by Lo et al. (2017) based on genetic evidence and is shared by a number of croaker ichthyologists but this conclusion needs further morphological support.

\section{Protonibea diacantha (Lacepède, 1802)}

Lutjanus diacanthus Lacepède, 1802: 195, 240 (no locality). Holotype (unique): MNHN A5539 (1, dry half skin).

Johnius cataleus Cuvier, 1829: 173 (India). Lectotype: MNHN A-5539 (1, dry half skin). Cuvier specimens: MNHN A-5539 (1, dry). Based on Russel 1803: fig. 116, and Bola chaptis Buchanan. Lectotype selected by Kottelat 2013: 359.

Corvina platycephala Kuhl and van Hasselt in Cuvier and Valenciennes, 1830: 132 (Java, Indonesia). No types known.

Johnius valenciennii Eydoux and Souleyet, 1850: 159, Pl. 1 (fig. 2) (Near Macao, China). Holotype (unique): MNHN 0000-7623.

Sciaena goma Tanaka, 1915: 615 (Nagasaki fish market, Japan). Syntypes: ZUMT 6392 (1). Corvina nigromaculata Borodin, 1930: 53, Pl. 2 (fig. 2) (Sri Lanka and Saigon, Vietnam). Holotype (unique): VMM 497 not 512 (now at AMNH). 
Sciaena antarctica rex Whitley, 1935: 26 (Onslow, Western Australia). Syntypes: (2) whereabouts unknown.

Distribution: Persian Gulf east to Philippines and southern Japan south to Queensland, Australia.

\section{Genus Protosciaena Sasaki, 1989}

Protosciaena Sasaki, 1989: 128. Feminine. Type species Sciaena trewavasae Chao and Miller, 1975. Type by original designation.

Western Atlantic over soft bottoms; two species.

\section{Protosciaena bathytatos Chao and Miller, 1975}

Sciaena bathytatos Chao and Miller, 1975: 267, fig. 9 (Caribbean Sea off Colombia, 9³6' N, $76^{\circ} 22^{\prime} \mathrm{W}$, Oregon station, 5722, 280 fathoms). Holotype: USNM 211514. Paratypes: AMNH 33470 (1); ANSP 126874 (2); BMNH 1974.4.1.3 (1); CAS 29740 (1); FMNH 77047 (1); MCZ 49119 (1); UF 19889 (1), 231589 ex UMML 31589 (1); USNM 211578-81 (1, 3, 1, 2). Distribution: Caribbean Sea off Colombia: Panama, Colombia, Venezuela and Trinidad.

\section{Protosciaena trewavasae (Chao and Miller, 1975)}

Sciaena trewavasae Chao and Miller, 1975: 262, fig. 8 (Caribbean Sea, off Colombia, $11^{\circ} 8^{\prime} 30^{\prime \prime} \mathrm{N}, 74^{\circ} 29^{\prime} \mathrm{W}$, Oregon station 4843, depth 100-120 fathoms). Holotype: USNM 211513. Paratypes: AMNH 33469 (1); ANSP 126873 (2); BMNH 1974.4.1.1-2 (2); CAS 29739 (1); FMNH 77046 (1); MCZ 49118 (1); UF 19888 (1), 23588 ex UMML 31588 (2); USNM 211573-76 (5, 3, 10, 3).

Distribution: Caribbean Sea, western Colombia to central Venezuela and from Puerto Rico.

Genus Pseudolarimichthys Lo, Liu, Mohd Nor and Chen, 2017

Pseudolarimichthys Lo, Liu, Mohd Nor and Chen, 2017: 9. Masculine. Type species Larimichthys terengganui Seah, Hanafi, Mazlan and Chao, 2015. Type by original designation.

West Pacific; monotypic.

\section{Pseudolarimichthys terengganui (Seah, Hanafi, Mazlan and Chao, 2015)}

Larimichthys crocea (nec Richardson, 1846), Matsunuma et al., 2011: 140 (Terengganu).

Larimichthys terengganui Seah, Hanafi, Mazlan and Chao, 2015: 272, figs. 1-4 (Fish landing port at Pulau Kambing, Kuala Terengganu, Malaysia). Holotype: NMMB-P 21523. Paratypes: KAUM-I. 16977, 1, Kuala Terengganu; NMMB-P 21723, 1 Pulau Kambing; NMMB-P 21542, 2 Pulau Kambing; NMMB-P 21524, 1; UMTF 05949, 05950, 2; UMTF 05951- 05956, 6.

Distribution: South China Sea around Kuala Terengganu, east coast of Peninsular Malaysia.

\section{Genus Pseudotolithus Bleeker, 1863}

Pseudotolithus Bleeker, 1863b: 59. Masculine. Type species Pseudotolithus typus Bleeker, 1863. Type by use of typus.

Pinnacorvina Fowler, 1925: 4. Feminine. Subgenus of Johnius, type species Rhinoscion epipercus Bleeker, 1863. Type by original designation.

FonticulusTrewavas, 1962: 169. Masculine. Subgenus of Pseudotolithus type species Corvina nigrita Cuvier, 1830. Type by original designation.

Hostia Trewavas, 1962: 170. Feminine. Subgenus of Pseudotolithus type species Corvina moorii Günther, 1865. Type by original designation.

Eastern Atlantic over mud and sandy bottoms from shoreline to $70 \mathrm{~m}$ of depth, rarely in deeper waters. Six species. 


\section{Pseudotolithus brachygnathus Bleeker, 1863}

Pseudotolithus brachygnathus Bleeker, 1863b: 62, Pl. 24 (fig. 2) (Ashantee, Guinea). Holotype (unique): RMNH 671. Holotype figured by Boeseman (1963: Pl. 4, fig. 3).

Sciaena sauvagei Rochebrune, 1882: 161 (Senegal). Syntypes: MNHN A-8146 (dry).

Distribution: Mauritania to Angola.

\section{Pseudotolithus elongatus (Bowdich, 1825)}

Sciaena elongata Bowdich, 1825: 236, fig. 43 (Porta Praya, St. Jago I., Madeira). Holotype (unique): not preserved.

Corvina clavigera Cuvier in Cuvier and Valenciennes, 1830: 101 (Senegal). Holotype (unique): MNHN 0000-6336 (poor condition).

Corvina nigrita Cuvier in Cuvier and Valenciennes, 1830: 103, fig. 138 (part) Senegal. Holotype (unique): MNHN 0000-7483 (poor condition).

Otolithus guineensis Osório, 1909: 102, Pl. (fig. 1) (Postugo, Bolama, Guinea-Bissau). Holotype (unique): MD (destroyed in 1978 fire).

Distribution: Senegal to southern Angola entering estuaries.

\section{Pseudotolithus epipercus (Bleeker, 1863)}

Rhinoscion epipercus Bleeker, 1863b: 64, Pl. 14 (Ashantee, Guinea). Holotype (unique): RMNH 687.

Corvina nigripinnis Günther, 1874: 453 (Cameroon coast). Holotype (unique): BMNH 1866.6.26.7.

Distribution: Guinea to southern Angola.

\section{Pseudotolithus moorii (Günther, 1865)}

Corvina moorii Günther, 1865: 48 (Gambia R. at Bathurst, Gambia). Holotype (unique): BMNH (whereabouts unknown).

Corvina camaronensis Ehrenbaum, 1915: 42 (West Africa). Syntypes: ZMH H4245 (1)

Cameroon.

Distribution: Gambia to southern Angola.

Comments: Taxonomic decision of Chao and Trewavas (1990).

\section{Pseudotolithus senegalensis (Valenciennes 1833)}

Otolithus senegalensis Valenciennes in Cuvier and Valenciennes, 1833: 476 (Gorée, Senegal). Syntypes: MNHN 0000-7512 (1), 0000-7514 (1).

Pseudotolithus macrognathus Bleeker, 1863b: 61, Pl. 13 (fig. 2) (Ashantee, Guinea). Holotype (unique): RMNH 753.

Distribution: Eastern tropical Atlantic from Morocco to Angola including Cape Verde Islands and São Tomé and Príncipe Islands (Wirtz et al., 2007).

\section{Pseudotolithus senegallus (Cuvier, 1830)}

Corvina senegalla Cuvier in Cuvier and Valenciennes, 1830: 132 (Senegal). Holotype (unique): MNHN 0000-7520.

Sciaena senegalensis Günther, 1860: 290 (Senegal). Holotype (unique): MNHN 0000-7520. Unjustified emendation of Corvina senegalla Cuvier, 1829.

Distribution: Senegal to southern Angola including Cape Verde Islands and São Tomé and Príncipe Islands.

Comments: Considered species dubia by Trewavas (1962), a possible synonym of $P$. brachygnathus Bleeker (Chao and Trewavas, 1990); a valid species according to Sasaki (1993).

\section{Pseudotolithus typus Bleeker, 1863}

Pseudotolithus typus Bleeker, 1863b: 60, Pl. 15 (fig. 1) (Ashantee, Guinea). Holotype (unique): RMNH 752. 
Sciaena dux Bowdich, 1825: 236, fig. 54 (Gambia R., w. Africa). No types known.

Distribution: Morocco to Angola including Cape Verde islands.

\section{Genus Pteroscion Fowler, 1925}

Pteroscion Fowler, 1925: 4. Masculine. Subgenus of Larimus, type species Larimus peli Bleeker, 1863. Type by original designation.

Eastern tropical Atlantic over mud and sandy bottoms from shoreline to $200 \mathrm{~m}$ of depth; monotypic.

\section{Pteroscion peli (Bleeker, 1863)}

Larimus peli Bleeker, 1863b: 63, Pl. 16 (fig. 2) (Ebriakwa, Guinea). Syntypes: RMNH 5970 (2).

Distribution: Senegal to Angola.

Genus Pterotolithus Fowler, 1933

Pterotolithus Fowler, 1933: 354, 359. Masculine. Subgenus of Otolithes, type species Otolithus maculatus Cuvier, 1830. Type by original designation.

Southeast Asia: found in coastal waters, estuaries and mouths of rivers; two species.

\section{Pterotolithus lateoides (Bleeker, 1849)}

Otolithus lateoides Bleeker, 1849b: 16 (Batavia [Jakarta], Indonesia). Syntypes: RMNH 5990 (3).

Otolithus dolorosus Seale, 1910: 280, Pl. 3 (Sandakan, Sabah state, Borneo, East Malaysia). Holotype (unique): BSMP 2485.

Distribution: Borneo, Indonesia, and Malaysia.

\section{Pterotolithus maculatus (Cuvier, 1830)}

Otolithus maculatus Cuvier (ex Kuhl and Van Hasselt) in Cuvier and Valenciennes, 1830: 64 (Batavia [Jakarta], Indonesia). No types known.

Otolithus bispinosus Cuvier in Cuvier and Valenciennes, 1830: 65 (Rangoon, Myanmar [Burma]). Holotype (unique): MNHN 0000-7619.

Otolithus submaculatus Blyth, 1860: 141 (Sitang R., Myanmar [Burma]). Syntypes: (2) whereabouts unknown.

Distribution: Northeastern India to Borneo.

\section{Genus Robaloscion Béarez and Schwarzhans, 2014}

Robaloscion Béarez and Schwarzhans, 2014: 273. Masculine. Type species Sciaena wieneri Sauvage 1883 . Type by original designation.

\section{Robaloscion wieneri (Sauvage, 1883)}

Sciaena wieneri Sauvage, 1883: 156 (Peru). Holotype (unique): MNHN A-4852.

Sciaena gilberti Starks, 1906: 794, Pl. 66 (fig. 3) (Callao, Peru). Nomen praeoccupatum, objectively invalid, preoccupied by Sciaena gilberti (Abbott, 1899). Callao, Peru. Holotype: USNM 53464. Paratypes: CAS-SU 9589 (1).

Sciaena starksi Evermann and Radcliffe, 1917: 104. Nomen novum, replacement name for Sciaena gilberti Starks, 1906. Holotype: USNM 53464. Paratypes: CAS-SU 9859 (1)

Distribution: Peru and northern Chile.

\section{Genus Roncador Jordan and Gilbert, 1880}

Roncador Jordan and Gilbert, 1880: 28. Masculine. Type species Corvina stearnsii Steindachner, 1876. Type by original designation.

Eastern Pacific in sandy shores and bays; monotypic. 


\section{Roncador stearnsii (Steindachner, 1875)}

Corvina stearnsii Steindachner, 1875b: 50 (San Diego, California, U.S.A.). Syntypes: (several) MCZ 18040 (3); NMW 14624 (1), 76994 (1).

Distribution: Punta Concepcion to Baja California.

\section{Genus Sciaena Linnaeus, 1758}

Sciaena Linnaeus, 1758: 288. Feminine.Type species Sciaena umbra Linnaeus, 1758. Type designated by ICZN (Opinion 988, supersedes Opinion 93); name placed on Official List.

Coracinus Pallas, 1814: 256. Masculine. Type species Coracinus chalcis Pallas, 1814. Type by subsequent designation. Type designated by Jordan 1917: 84.

Corvina Cuvier, 1829: 173. Feminine. Type species Sciaena nigra Forsskål, 1775. Type by subsequent designation of Gill, 1862: 85. Nomen preoccupatum, preoccupied by Corvina Hahn, 1822 in Aves, Campephagidae, a junior synonym of Coracina Viellot, 1816.

Melantha Gistel, 1848: 109. Feminine. Type species Sciaena nigra Forsskål, 1775 (= Sciaena umbra Linnaeus 1758). Type by being a replacement name. Replacement for Corvina Cuvier, 1829.

Excursor Gistel, 1848 XIII. Masculine. Type species Corvina nigra of Cuvier 1829 (= Sciaena umbra Linnaeus 1758). Type by being a replacement name. Unneeded replacement for Melantha Gistel 1848 thought to be preoccupied by Melanthia Duponchee, 1829 in Geometridae, Lepidoptera

Western Atlantic, eastern Atlantic and Mediterranean to Black Sea; the eastern Pacific species should be assigned to other genera (Chao, unpublished). In his early studies Chao (1978) recognized only three valid species (bathytatos, trewavase, and umbra), but the genus was not well-defined and it was supposed to contain more species. Two species are listed here. New genera have been erected for $S$. trewavase (see Protosciaena) and $S$. wieneri (see Robaloscion).

\section{Sciaena callaensis Hildebrand, 1946}

Sciaena callaensis Hildebrand, 1946: 287, fig. 61 (Callao Bay, Peru). Holotype: USNM 36934. Paratypes: USNM 120709 ex USNM 36934 (1).

Distribution: Eastern Pacific: off Peru.

\section{Sciaena umbra Linnaeus, 1758}

Sciaena umbra Linnaeus, 1758: 289 (Zara, Dalmatia, Yugoslavia, Mediterranean Sea). Neotype: BMNH 1893.9.21.10. Neotype designated by Trewavas 1966: 5.

Sciaena nigra Bloch, 1792: 35, Pl. 297 (Mediterranean Sea). Syntypes: ZMB 8739 (1, stuffed). Nomen preoccupatum, objectively invalid, preoccupied by Sciaena nigra Forsskål, 1775 .

Coracinus chalcis Pallas, 1814: 256 (Ponto, Alupka). No types known.

Corvina canariensis Cuvier in Cuvier and Valenciennes, 1830: 93 (Canary Islands). Holotype (unique): MNHN 0000-7609 (dry).

Distribution: Mediterranean Sea, Black Sea and eastern Atlantic from English Channel to Senegal and possibly Gabon, including Canary and Cape Verde islands.

Comments: Taxonomic decision of Trewavas (1973) and Chao and Trewavas (1990).

\section{Genus Sciaenops Gill, 1863}

Sciaenops Gill, 1863a: 30. Masculine. Type species Perca ocellata Linnaeus, 1766. Type by monotypy.

Western Atlantic, usually over sand and sandy mud bottoms in coastal waters and estuaries; abundant in surf zone; monotypic. 
Sciaenops ocellatus (Linnaeus, 1766)

Perca ocellata Linnaeus (ex Garden), 1766: 483 (Carolina South Carolina, U.S.A.). Syntypes: LS 106-107 (2, right half-skins).

Lutjanus triangulum Lacepède, 1802: 181 (Eastern U.S.A.). No types known.

Sciaena imberbis Mitchill, 1815: 411 (New York, U.S.A.). No types known.

Distribution: Massachusetts in USA to northern Mexico, including southern Florida, USA.

\section{Genus Seriphus Ayres, 1860}

Seriphus Ayres, 1860: 80. Masculine. Type species Seriphus politus Ayres, 1860. Type by monotypy.

Kroseriphus Whitley, 1950: 44. Masculine. Type species Seriphus politus Ayres 1860. Type by being a replacement name. Unneeded replacement name for Seriphus Ayres, 1860 thought to be preoccupied by Seripha Walker, 1854 in Arctiidae, Noctuoidea, Lepidoptera,

Eastern Pacific, inshore, often over sandy bottoms in bays and tidal sloughs, around pilings, moving to deeper water at night; monotypic.

\section{Seriphus politus Ayres, 1860}

Seriphus politus Ayres, 1860: 80, fig. 21 (San Francisco Bay, California, U.S.A.). Syntypes: (2 or 3) whereabouts unknown.

Distribution: Yaquina Bay, Oregon to southern Baja California; also recorded from British Columbia, Canada.

\section{Genus Sonorolux Trewavas, 1977}

Sonorolux Trewavas, 1977: 389 Feminine.Type species Sonorolux fluminis Trewavas, 1977. Type by original designation.

West Pacific, in estuaries; monotypic.

\section{Sonorolux fluminis Trewavas, 1977}

Sonorolux fluminis Trewavas, 1977: 390, fig. 32 (Sarawak state, Borneo, East Malaysia). Holotype: BMNH 1895.2.28.47. Paratypes: BMNH 1905.11.16.9 (1).

Distribution: Sarawak, Borneo.

\section{Genus Stellifer Oken, 1817}

Stellifer Oken (ex Cuvier), 1817: 1182. Masculine. Type species Bodianus stellifer Bloch, 1790. Type by monotypy.

Stelliferus Stark, 1828: 459. Masculine. Type species Stelliferus capensis Stark, 1828 (= Bodianus stellifer Bloch, 1790). Type by monotypy.

Homoprion Holbrook, 1855: 168. Masculine. Type species Homoprion lanceolatus Holbrook, 1855. Type by monotypy.

Nector Jordan and Evermann, 1898: 1432, 1436. Masculine. Type species Corvina chrysoleuca Günther 1867 . Type by original designation.

Stellicarens Gilbert in Jordan and Evermann, 1898: 1440. Masculine. Subgenus of Stellifer, type species Stellifer zestocarus Gilbert, 1898. Type by original designation.

Zestidium Gilbert in Jordan and Evermann, 1898: 1439. Neuter. Subgenus of Stellifer, type species Stellifer illecebrosus Gilbert, 1898. Type by original designation.

Zestis Gilbert in Jordan and Evermann, 1898: 1439. Feminine. Subgenus of Stellifer, type species Sciaena oscitans Jordan and Gilbert, 1882. Type by original designation.

Amphi-American, inhabiting inshore waters of sandy bottoms, estuaries and around coral reefs. Twenty-four species, 13 of which are in the eastern Pacific and 11 in the western Atlantic. Four species are still undescribed. 


\section{Stellifer brasiliensis (Schultz, 1945)}

Ophioscion brasiliensis Schultz, 1945: 126, 128, fig. 6 (Sand bar at Barro Santos, Brazil). Holotype: USNM 87742. Paratypes: USNM 122611 (1).

Distribution: Brazil.

\section{Stellifer chaoi Aguilera, Solano and Valdez, 1983}

Stellifer chaoi Aguilera, Solano and Valdez, 1983: 7, fig. 1 (Punta Campana, Golfo de Venezuela, depth 8-10 fathoms). Holotype: MBUCV-V-13815. Paratypes: ANSP 153376 (6); CIP 608 (3); ICNMNH 843 (4); INVEMAR P-719 (6), P-722 (3), P-729 (2), P-765 (3); MBUCV-V-14030 (35), MM 5069 PEC 1448 (3); UF 234426 ex UMML 34426 (6); UNEFM 13815 (35).

Distribution: South Caribbean Sea.

\section{Stellifer chrysoleuca (Günther, 1867)}

Corvina chrysoleuca Günther, 1867: 600 (Pacific Panama). Syntypes: BMNH 1865.7.20.24 (1), 1866.1.14.7 (1). Described in more detail in Günther 1868: 427, Pl. 67 (fig. 1).

Sciaena aluta Jordan and Gilbert, 1882b: 232 La Union, Gulf of Fonseca, El Salvador, eastern Pacific. Holotype (unique): USNM 28129.

Distribution: Mexico to Peru.

\section{Stellifer colonensis Meek and Hildebrand, 1923}

Stellifer colonensis Meek and Hildebrand, 1923: 623, Pl. 66 (fig. 1) (Mindi Reef, Mindi, Canal Zone, Panama). Holotype: USNM 81729. Paratypes: (41) FMNH 18576-77 (2), 1857883 (6), 18584-92 (8), 18593-94 (4); USNM 81217 (2), 81220 (2), 81236 (3), 81326-27 (6, 1). Distribution: Central western Atlantic.

\section{Stellifer ephelis Chirichigno, 1974}

Stellifer ephelis Chirichigno, 1974: 252, 350, fig. 470 (type localities, Golfo de Fonesco and Caleta Cruz, Peru). Holotype is the specimen in the illustration (fig. 470)

Stellifer sp. 2. Chao, 1995: 1443.

Distribution: Eastern Pacific: Pacific coast of Central America from the Gulf of Fonseca, Honduras, to northern Peru.

Comments: This species refers to the manuscript name Stellifer ephelis Wintersten who had a publication with Chao but it was never published; Chirichigno took the name from the manuscript by Wintersteen, but apparently Chirichigno was responsible for the description and figure, so she is regarded as author; types would be specimens seen by her; the name $S$. ephelis is therefore regarded as available (Eschmeyer, 1998).

\section{Stellifer ericymba (Jordan and Gilbert, 1882)}

Sciaena ericymba Jordan and Gilbert, 1882a: 311 (Bay of Panama, Pacific Panama). Syntypes: USNM 29338 (1, lost), 29433 (1, lost), 29466 (1), 29477 (1, lost), 29479 (1, lost), 29494 (1).

Stellifer erycimba peruana Hildebrand, 1946: 305, fig. 66 (Gulf of Guayaquil, off Puerto Pizarro, Peru. Holotype: USNM 128038. Paratypes: USNM 128039 (9), 128040 (14); Gov't of Peru (1).

Distribution: Central Mexico to northern Peru.

\section{Stellifer fuerthii (Steindachner, 1875)}

Corvina (Homoprion) fuerthii Steindachner, 1875b: 54, Pl. 3 (Panama Bay, Pacific Panama). Syntypes: (several) NMW 78575 (2).

Distribution: Honduras to Peru.

\section{Stellifer griseus Cervigón, 1966}


Stellifer griseus Cervigón, 1966: 1, figs. 1-2 (Morro de Puerto Santo, Estado Sucre, Venezuela, depth 6 meters). Holotype: MOBR-EDIMAR-P-1875. Paratypes: MOBREDIMAR -P- 347 (3), 349 (7), 1876 (4); USNM 200782 (2).

Distribution: Along the Atlantic coasts of Colombia, Venezuela, and Trinidad and Tobago.

\section{Stellifer illecebrosus Gilbert, 1898}

Stellifer illecebrosus Gilbert in Jordan and Evermann, 1898: 1442 (San Jose Rock, Bay of Panama). Lectotype: ? CAS-SU 5515. Paralectotypes: (2) CAS-SU 6907 (1).

Distribution: Mexico to Peru.

Stellifer lanceolatus (Holbrook, 1855)

Homoprion lanceolatus Holbrook, 1855: 168, Pl. 23 (fig. 1) (Very deep water, South Carolina, U.S.A.). No types known.

Sciaena stellifera Jordan and Gilbert, 1883: 569 (nec Bodianus stellifer, Bloch).

Distribution: Virginia to Texas except southern Florida.

\section{Stellifer magoi Aguilera, 1983}

Stellifer magoi Aguilera, 1983b: 148, fig. 1 (Caño Guanoco, cerca de Guanoco en la Cuenca del Golfo de Paria, Venezuela). Holotype: MBUCV-V-12376. Paratypes: MBUCV-V-13615 (4).

Distribution: Venezuela.

\section{Stellifer mancorensis Chirichigno, 1962}

Stellifer mancorensis Chirichigno, 1962: 17, fig. 16 (Máncora, Peru). Holotype: MHNJP = MUSM P 1248. Paratypes: ?MHNJP = MUSM P and C 446 (1).

Distribution: Costa Rica to Peru.

\section{Stellifer melanocheir Eigenmann, 1918}

Stellifer melanocheir Eigenmann, 1918: 682 (Tumaco, Colombia). Holotype (unique): FMNH 58507 ex CM 7520.

Distribution: Colombia (Eastern Pacific).

\section{Stellifer microps (Steindachner, 1864)}

Corvina stellifera Günther, 1860: 299 (nec Bodianus stelliferus Bloch).

Corvina microps Steindachner, 1864: 205, Pl. 2 (fig. 1) (Guyana). Syntypes: (several) ?NMW.

Ophioscion costaricensis Caldwell, 1958: 117, fig. 1 (Tortuguero, Caribbean Costa Rica, $10^{\circ} 34^{\prime} \mathrm{N}, 83^{\circ} 32^{\prime} \mathrm{W}$, found on beach). Holotype: UF 5831. Paratypes: UF 5830 (1).

Distribution: Costa Rica to Brazil.

\section{Stellifer minor (Tschudi, 1846)}

Corvina minor Tschudi, 1846: 9 (Market at Lima, Peru). Syntypes: (several) ZMB 895 (1). Corvina (Homoprion) agassizi Steindachner, 1875a: 468 (Caldera; Callao; Paita, Peru). Syntypes: (several) NMW 14611-12 (2, 1), 15334 (2), 15456 (1), ?78574 (1), ?85306 (2).

Distribution: Peru and Chile.

Comment: Taxonomic decision of Hildebrand (1946).

\section{Stellifer naso (Jordan in Jordan and Eigenmann, 1889)}

Stelliferus naso Jordan in Jordan and Eigenmann, 1889: 395 (Cachiura, Brazil). Syntypes: USNM 130630 (5).

Distribution: Brazil.

\section{Stellifer oscitans (Jordan and Gilbert, 1882)}

Sciaena oscitans Jordan and Gilbert, 1882a: 312 (Pacific Panama). Syntypes: USNM 29258 (1), 29299 (1), 29319 (1), 29326 (1). 
Distribution: Honduras to Peru.

\section{Stellifer pizarroensis Hildebrand, 1946}

Stellifer pizarroensis Hildebrand, 1946: 304, fig. 65 (Gulf of Guayaquil, off Puerto Pizarro, Peru). Holotype: USNM 128036. Paratypes: USNM 128037 (1).

Distribution: Peru.

\section{Stellifer rastrifer (Jordan, 1889)}

Stelliferus rastrifer Jordan in Jordan and Eigenmann, 1889: 393 (Santos, São Paulo State, Brazil). Holotype: MCZ 10815. Paratypes: MCZ 4582 (2), 10802 (3), 10807 (13), 10811 (1), 10813-14 (5, 1), 10816-17 (15, 1), 10822 (6), 36561 (4), 91347 (13), 91348 ex MCZ 10815 (1); USNM 130631 (5), 153609 ex MCZ 10817 (5).

Distribution: Brazil.

\section{Stellifer stellifer (Bloch, 1790)}

Bodianus stellifer Bloch, 1790: 55, Pl. 231 (fig. 1) (Cape of Good Hope, South Africa). Holotype (unique): ZMB 885.

Stelliferus capensis Stark, 1828: 459 (Cape of Good Hope). No types known. Nomen novum, unneeded replacement name for Bodianus stellifer Bloch, 1790.

Corvina trispinosa Cuvier, 1829: 109 (South Africa). Cuvier specimens: MNHN 0000-5428 (1) Brazil, 7620 (1) Cayenne, A-5544 (2, dry) Brazil.

Stellifer mindii Meek and Hildebrand, 1925: 626, Pl. 66 (fig. 2) (Mindi Reef, Mindi, Canal Zone, Panama). Holotype: USNM 81730. Paratypes: FMNH 18573-75 (3).

Distribution: Atlantic coast of Panama to Brazil.

\section{Stellifer venezuelae (Schultz, 1945)}

Ophioscion venezuelae Schultz, 1945: 131, fig. 7 (Near mouth of Caño de Sagua, 25 kilometers north of Sinamaica, Venezuela). Holotype: USNM 121749. Paratypes: FMNH 62622 ex USNM 121570 (1), MCZ 37217 ex USNM 121750, USNM 121750 (orig. 6, now 4).

Distribution: Western Atlantic.

\section{Stellifer walkeri Chao, 2001}

Stellifer sp. 3. Chao, 1995: 1442.

Stellifer walkeri Chao, 2001: 68, figs. 1-3 (Bahia Matenchen in vicinity of San Blas, Nayarit, Mexico, $21^{\circ} 30^{\prime} \mathrm{N}, 105^{\circ} 30^{\prime} \mathrm{W}$, depth 2.7-13.5 meters). Holotype: USNM 208556. Paratypes: CAS 16051 (3); LACM 58-40 (7); MCZ 156816 (1); NMC 68-1380 (1); SIO 13-238 (2) (ex UCLA W58-38), 23-52 (2), 62-37 (1); UNAM 8900 (2); USNM 208557 (7).

Distribution: Eastern Pacific: central coast of Mexico.

\section{Stellifer wintersteenorum Chao, 2001}

Stellifer sp. 1. Chao, 1995: 1442.

Stellifer wintersteenorum Chao, 2001: 71, figs. 4-6 (Bahia Matenchen in vicinity of San Blas, Nayarit, Mexico, depth to 13.7 meters). Holotype: USNM 208559. Paratypes: ANSP 122063 (40); BMNH 1973.6.1.20 (20); CAS 16052 (80); FMNH 71706 (40); LACM 50-43 (1), 56123 (2), 56-148 (1), 58-31 (87), 58-33 (27), 58-39 (43), 58-40 (8), 6545-1 (1); NMC 68-111 (12); SIO 60-87 (4), 62-71 (4), 62-72 (2), 64-375 (1), 65-104 (1), 73-51 (40); UCLA 58-31 (22), 58-34 (35), 58-35 (11); UNAM 8901 (10); USNM 208560 (60).

Distribution: Eastern shore of the Gulf of California.

\section{Stellifer zestocarus Gilbert, 1898}

Stellifer zestocarus Gilbert in Jordan and Evermann, 1898: 1445 (Panama Bay, Panama). Lectotype: CAS-SU 5518. Paralectotypes: BMNH 1903.5.15.144 (1), CAS-SU 6908 (3), 
?ZMB 15980 [ex SU] (1). Also appeared as Gilbert in Gilbert and Starks, 1904: 129, Pl. 18 (fig. 37) with lectotype established by use of "type specimen" in caption to figure 37.

Distribution: Gulf of California.

\section{Genus Totoaba Villamar, 1980}

Totoaba Villamar, 1980: 130. Feminine. Type species Cynoscion macdonaldi Gilbert, 1890. Type by original designation.

Eastern Pacific, in coastal waters; juveniles in river mouths and near rocky coasts; monotypic.

\section{Totoaba macdonaldi (Gilbert, 1890)}

Cynoscion macdonaldi Gilbert, 1890: 64 (Eastern shore of Gulf of California and near the mouth of Colorado R., north Gulf of California, Mexico). Syntypes: USNM 43082 (1).

Distribution: Gulf of California.

Comments: This is the giant of the sciaenid fishes: up to $200 \mathrm{~cm}$ and $100 \mathrm{Kg}$. Due to overfishing the species is classified as critically endangered by the IUCN that reports "A total ban on fishing was declared by the Mexican Government in 1975 and this species was placed on the Mexican Endangered Species List [...] However, illegal fishing for this species in spawning grounds continued for several years after these conservation measures were enacted, and was only brought under effective control in the 1990s."

\section{Genus Umbrina Cuvier, 1816}

Umbrina Cuvier, 1816b: 297. Feminine. Type species Sciaena cirrosa Linnaeus, 1758. Type by monotypy, only one species included. On Official List (Opinion 988).

Attilus Gistel, 1848: 109. Masculine. Type species Sciaena cirrosa Linnaeus, 1758. Type by being a replacement name. An unneeded replacement for Umbrina Cuvier 1816.

Asperina Ostroumoff, 1896: 30. Feminine. Type species Asperina improvisa Ostroumoff, 1896. Type by monotypy.

Worldwide in inshore and offshore waters with sandy or muddy bottoms and around coral reefs; 17 species (Sasaki, 1989; Walker and Radford, 1992).

\section{Umbrina analis Günther, 1868}

Umbrina analis Günther, 1868: 426 (Pacific Panama). Holotype (unique): BMNH 1867.9.23.18.

Umbrina tumacoensis Wilson, 1916: 67, Pl. 10 (Tumaco, Department of Nariño, Colombia). Holotype: FMNH 56840 ex CM 5654 (missing). Paratypes: CAS 62852 IU 13438 b-c (2), FMNH 56840 ex CM 5654b-c (2).

Distribution: Eastern Pacific: Mexico to northern Ecuador.

Comments: Taxonomic decision of Walker and Radford (1992).

Umbrina broussonnetii Cuvier, 1830

Umbrina broussonnetii Cuvier in Cuvier and Valenciennes, 1830: 187 (Jamaica). Holotype (unique): MNHN 0000-7471.

Distribution: Costa Rica to Colombia and Greater Antilles.

\section{Umbrina bussingi Lopez, 1980}

Umbrina bussingi Lopez, 1980: 203, fig. 1 (Costa Rica, e. Pacific, 902' N, 84²13' W, 183290 m). Holotype (unique): LACM 38715-1.

Distribution: Eastern Pacific: southern Baja California to Ecuador.

\section{Umbrina canariensis Valenciennes, 1843}

Umbrina canariensis Valenciennes, 1843: 24 (Canary Islands). Syntypes: MNHN A-5669 (1, dry), 7608 (1, dry). 
Umbrina lafonti Moreau, 1874: 118, Pl. 14 (Gulf of Gascony, France). Syntypes: MNHN 1898-0568 (1), 7307 (1).

Umbrina sinuata Day, 1876: 182, Pl. 46 (fig. 1) (Karachi, Pakistan). Syntypes (2) and/or Day specimen: ZSI 939 (1, fragile).

Umbrina striata Boulenger, 1888: 660 (Muscat, Oman, Gulf of Oman, Arabian Sea, northwestern Indian Ocean). Holotype (unique): BMNH 1887.11.11.164 (stuffed).

Umbrina valida Jordan and Gunn, 1898: 342 (Canary Islands). Holotype (unique): CAS-SU 10584.

Distribution: Western Indian Ocean, eastern Atlantic.

Umbrina canosai Berg, 1895

Umbrina canosai Berg, 1895: 56 (Atlantic near Montevideo, Uruguay, and Mar del Plata). Syntypes: MACN 5081 (1), MHNM.

Distribution: Montevideo (Uruguay) to Mar del Plata, Argentina.

\section{Umbrina cirrosa (Linnaeus, 1758)}

Sciaena cirrosa Linnaeus, 1758: 289 (Mediterranean Sea). No types known.

Sciaena umbra Linnaeus, 1758 partim.

Sciaena discolor Walbaum, 1792: 319 (Suriname or Mediterranean Sea). No types known.

Sparus coracinus Asso, 1801: 36 (Spain). No types known.

Cheilodipterus cyanopterus Lacepède, 1801: 540, 546, Pl. 16 (fig. 3) (Cayenne, French Guiana). No types known.

Anthias rubescens Bloch and Schneider, 1801: 307 (Suriname rivers). Based on Gronow Zoophylacium. No. 212, later described as Sciaena cestreus Gronow in Gray, 1854.

Perca umbra Lacepède, 1802: 396, 414 (Mediterranean Sea; Antilles; etc.). No types known. Nomen novum, unnecessary replacement name for Sciaena cirrosa Linnaeus, 1758.

Coracinus boops Pallas, 1814: 259 (Ponti Euxini, Tauriae, Black Sea off Feodosiya, Crimea). No types known.

Umbrina cirrhata Geoffroy Saint Hilaire, 1809: 321, pl. 22 (figs. 1 and 1') (Mediterranean Sea). No type known.

Umbrina vulgaris Cuvier in Cuvier and Valenciennes, 1830: 171 (Provence, France; Italy; Spain; Gulf of Gascony). Syntypes: MNHN 0000-7463 (1) Iviça, 0000-7466 (1) Morée; nontypes MNHN 0000-7462 (1) Naples, 0000-1962 (1) Egypt.

Sciaena cestreus Gronow in Gray, 1854: 52 (Mediterranean Sea, ?American seas). Syntypes: BMNH 1853.11.12.74 Gronovius coll. (1, skin).

Asperina improvisa Ostroumoff, 1896: 30 (Temryuk, mouth of Kuban River, Sea of Azov, Russia). No types known.

Distribution: Eastern Atlantic, Mediterranean, Black Sea and Red Sea immigrant.

Comments: Taxonomic decisions of Wheeler (1958) and Trewavas (1973).

\section{Umbrina coroides Cuvier, 1830}

Umbrina coroides Cuvier in Cuvier and Valenciennes, 1830: 187, Pl. 117 (Brazil). Holotype (unique): MNHN 0000-5343.

Distribution: Bahamas and Texas to Brazil but absent from northern and eastern Gulf of Mexico.

\section{Umbrina dorsalis Gill, 1862}

Umbrina dorsalis Gill, 1862b: 257 (Baja California, Mexico). Lectotype: USNM 3696. Paralectotypes: USNM 316654 ex USNM 3696 (7). Lectotype selected by Walker and Radford (1992: 580).

Distribution: Eastern Pacific, Mexico to Ecuador. 


\section{Umbrina galapagorum Steindachner, 1878}

Umbrina galapagorum Steindachner, 1878a: 396 (James I., Galápagos Islands). Lectotype: MCZ 8601. Paralectotypes: NMW 78601 (2), 78632 (1), $84792-93$ (1, 1), 84796-97 (2, 2), 85690 (1), 86244 (2); USNM 120437 ex MCZ 8601 (1), 153626 ex MCZ 8597 (1). Lectotype selected by Walker and Radford (1992: 581).

Distribution: Galápagos Islands.

\section{Umbrina milliae Miller, 1971}

Umbrina milliae Miller, 1971: 303, figs. 1-3 (Off Colombia, South America, $11^{\circ} 08^{\prime} \mathrm{N}, 7^{\circ}$ 23.8' West Oregon station 4856, depth about 100 fathoms). Holotype: USNM 204932. Paratypes: UF 19287 ex TABL 107368 (1).

Distribution: Colombian Caribbean.

\section{Umbrina reedi Günther, 1880}

Umbrina reedi Günther, 1880: 25, Pl. 13 (fig. B) (Juan Fernández Islands). Syntypes: BMNH 1874.1.10.4 (1), 1879.5.14.283 (1).

Distribution: Juan Fernández Islands, Desventuradas Islands (Chile).

\section{Umbrina robinsoni Gilchrist and Thompson, 1908}

Umbrina robinsoni Gilchrist and Thompson, 1908: 182 (Natal, South Africa). Holotype (unique): SAM 9966.

? Umbrina capensis Pappe, 1853: 16 (False Bay, South Africa).

Distribution: Southern Africa.

Comments: Resurrected from synonymy with $U$. ronchus by Heemstra and Heemstra (2004: 272). Umbrina capensis has been regarded as nomen dubium as it cannot be assigned to either of the two species known from South Africa according to Heemstra (1986). Treated as a possible synonym of Umbrina ronchus Valenciennes, 1843 by Trewavas (1973: 401) or as a doubtful synonym of $U$. robinsoni according to Hutchings and Griffiths (2005: 14).

\section{Umbrina roncador Jordan and Gilbert, 1882}

Umbrina roncador Jordan and Gilbert, 1882c: 277 (Pequeña Bay, w. coast of Baja California, Mexico). Holotype (unique): USNM 29371.

Sciaena thompsoni Hubbs, 1921: 1, Pl. 1 (Avalon, Santa Catalina Island, California, U.S.A.). Holotype: UMMZ 55053 ex FMNH 9098.

Distribution: Point Conception to Gulf of California.

Comments: Taxonomic decision of Walker and Radford (1992).

Umbrina ronchus Valenciennes, 1843

Umbrina ronchus Valenciennes, 1843: 24 (Canary Islands). Holotype (unique): MNHN A5764 (dry).

Umbrina angustilineata Gilchrist and Thompson, 1911: 38 (KwaZulu-Natal, South Africa). Holotype (unique): SAM 10559.

Umbrina fusca Dardignac in Furnestin, Dardignac, Vincent, Coupé and Boutière, 1958: 441, fig. 46 (Agadir and Casablanca, Morocco, eastern Atlantic). Syntypes: (4) ISTPM (apparently lost).

Distribution: Southwestern Mediterranean Sea, eastern Atlantic: southern Portugal south to Angola, including Canary and Cape Verde islands. Persian Gulf and western Indian Ocean to southern Africa.

Comments: Taxonomic decision of Chao and Trewavas (1990).

\section{Umbrina steindachneri Cadenat, 1951}

Umbrina steindachneri Cadenat, 1951: 221, fig. 156 (Senegal). No types known.

Distribution: Eastern Atlantic. 


\section{Umbrina wintersteeni Walker and Radford, 1992}

Umbrina wintersteeni Walker and Radford, 1992: 582, fig. 10 (Bahia Almejas, Baja California, Mexico). Holotype: SIO 60-366. Paratypes: AMNH 5498 (1), 5299 ex 5514a (1); CAS 35536 (4); LACM 38104-26 (4); SIO 15-622 (2) (ex UCLA W50-27), 15-1696 (5) (ex UCLA W52-48), 15-631 (6) (ex UCLA W53-84), 15-636 (8) (ex UCLA W53-95), 15-1136 (1) (ex UCLA W57-34), 15-1697 (4) (ex UCLA 57-36), 15-1698 (4) (ex UCLA W57-42), 60366 (29), 62-126 (10), 64-84 (49), 65-281 (3), 76-275 (2); CAS-SU 375 (1), 2855 (3), 4827 (1), 47933 (1); UCLA W52-49 (2), W62-50 (1); USNM 316655 (5).

Distribution: Eastern Pacific: southern Baja California, Mexico and the Gulf of California.

\section{Umbrina xanti Gill, 1862}

Umbrina xanti Gill 1862b: 257 (Baja California, Mexico). Lectotype: USNM 7156. Paralectotypes: MCZ 35976 [ex USNM 3693] (1); USNM 2296 (2), 3693 + 3694 (5), 316653 [ex USNM 7156] (2). Lectotype selected by Walker and Radford 1992: 577.

Umbrina sinaloae Scofield in Jordan, 1896: 220, Pl. 25 (Mazatlán, Sinaloa, western Mexico). Lectotype: CAS-SU 1632. Paralectotypes: CAS-SU 1632 (37, incl. lectotype). Lectotype established (as figured specimen) in Jordan, 1896: 244 if specimen is identifiable; also caption to P1. 224, p. 3279 in Jordan and Evermann, 1900 if figured specimen is identifiable.

Distribution: Mexico to Chile.

\section{Incertae sedis}

Lonchurus arcuatus Bloch and Schneider, 1801, Systema Ichthyol.: 103 (Suriname).

Lonchurus nasus Bloch and Schneider, 1801, Systema Ichthyol.: 103 (Suriname).

Nibea pacifica Hatai, 1965: 71, Pl. 15 (figs. 43, 44) (Japan).

Comments: Described on the basis of fossil otoliths; not related to living species; treated as a doubtful species by Trewavas (1977).

Plagioscion microps Steindachner, 1917a: 228 (Onça Island, Amazon River, Brazil). Syntypes: (2) ?NMW 15180 (1). Species illustrated and described in more detail in Steindachner, 1917b: 657, Pl. 1 (fig. 1).

Comments: This species falls within the genus Nebris according to Casatti (2005: 41) but it might belong to a new genus.

Plagioscion pauciradiatus Steindachner, 1917a: 228 (Salt and brackish water near Paramaribo, Suriname). Syntypes: (3) NMW. Species illustrated and described in more detail in Steindachner 1917b: 660, fig.

Comments: In the revision of the genus Plagioscion Casatti (2005: 41) wrote: "the types were not located and the original description is insufficient to allocate this species to any known species of Plagioscion."

Pogonathus auratus Lacepède, 1803: 120, 121 (no locality). No types known.

Comments: Based on an incomplete description made by Commerson; type material not available. A species not identifiable (Bauchot and Desoutter, 1987)

Pseudosciaena acanthodes Bleeker, 1879: 29, Pl. 1 (Japan). Holotype (unique): ZMH (lost). Comments: Not reported by Jordan and Tanaka (1902). A synonym of unidentified species of Pennahia.

Pseudotolithus bleekeri Steindachner, 1866d: 773, Pl. 14 (fig. 4) (Hong Kong, China). Holotype (unique): NMW.

Sciaena arcuata Basilewsky, 1855: 220 (Mari meridiano, Pelim, China). No types known. 
Sciaena brevidorsalis Günther, 1860: 295 (East Indies with question). Holotype (unique): BMNH 1855.9.19.388.

Sciaena pentadactyla Lacepède, 1802: 310, 316 (Mauritius).

Comments: A species not identifiable (Bauchot and Desoutter, 1987)

Sciaena peruana Steindachner, 1903: 203 (Coquimbo, Chile). Syntypes: ZMB 16290-91 (1, 1).

Comments: Status unknown (Béarez and Schwarzhans, 2014: 273). Using the key to genera given by Chao (1995) the species may belong to the genus Sciaena or Cheilotrema.

Sciaena rubella Jardine and Schomburgk in Schomburgk, 1943: 133 (Guyana Rivers). No types known.

Comments: Treated as a synonym of Plagioscion squamosissimus by Chao (1978: 44), its status remains doubtful according to Casatti (2005: 41) as the original description is insufficient to allocate this species to any known species of Plagioscion.

Sphenanthias dorianus Borodin, 1932: 80, Pl. 1 (fig. 1) (Southern entrance to Dorian Strait, Equator, Indonesia, depth 14 fathoms. Holotype: VMM 1184. Paratypes: MCZ 33057 ex VMM 1184 (1). Doubtful species, placement uncertain (Trewavas, 1977: 451).

Notes on some nominal species described in sciaenid genera but not belonging to Sciaenidae.

Corvina punctata Castelnau, 1861: 9 (Durban [Port Natal], South Africa). No types known.

Comments: This is a junior synonym of Pomadasys commersonnii (Lacepède, 1801), family Haemulidae. It is characterized by the following fin formula: D. XI/14, A III/9, V. I/5, P. 18, C. 17. Upper part of the body grey-greenish covered by many black-violet dots scattered in no order, a darker blotch on the posterior margin of the opercle; dorsal fin violet-blackish, pectorals whitish, ventral and anal fin white-yellowish; preopercle margin serrated; second anal spine very strong.

Cynoscion fusiformis Borodin, 1934: 115, Pl. 2 (fig. 1) (Florida Keys, Florida, U.S.A.). Holotype (unique): VMM 1267 (now at AMNH).

Comments: Not treated in the revision of the western Atlantic species prepared by Chao (1978) because regarded as not a sciaenid. Chao (2002: 8) stated: "A photo of the holotype resembles the Stizostedion species (Percidae)."

Johnius macrolepidotus Bloch and Schneider, 1801: 76 (Tranquebar, India). No type known. Comments: An haemulid; possibly Pomadasys guoraca (Cuvier, 1829).

Sciaena abuhamrur Walbaum, 1792: 310 (Red Sea).

Comments: An unidentifiable lethrinid.

Sciaena cappa Linnaeus, 1758: 288 (Mediterranean Sea).

Comments: Not a sciaenid, described with three anal spines. Description very short: "Sciaena capitis lateribus squamarum ordine duplici, D 11/23; P 16; V 1/6; A 3/13; C 17". Later mentioned by Linnaeus in the second part of the Museum Adolphi Friderici (Linnaeus, 1764: 81) where few additional details are added: "Oculi magni, dentes paralleli quasi pectinate. Corpus ovatum. Fossula recondens pinnam dorsi". Possibly a species of the genus Spicara, family Sparidae.

Sciaena dib Gmelin, 1789: 1305 (Arabia). No types known. Possibly a lethrinid.

Sciaena guttata Bloch and Schneider, 1801: 80 (Indian Ocean). No types known.

Comments: The fish has been described with the following meristics: Br.5; P.12; V.6; A-I/8; D VII/VII, 9. Description also included: "Head long, naked, tail elongated, fragile, rounded black spot, in two series, fins black spotted, anus in medial position, fins reduced." Sciaena 
guttata represents the eleotrid species presently known as Giuris margaritaceus (Valenciennes, 1837). This is the third species that Bloch and Schneider placed in the genus Sciaena that belong to the Eleotridae, the others being Sciaena macrolepidota (Bloch, 1792) [= Ophiocara macrolepidota (Bloch, 1792)] and Sciaena maculata Block, 1792) [=Dormitator maculatus (Bloch, 1792)]. Sciaena guttata Bloch and Schneider, 1801 predates Eleotris margaritacea Valenciennes, 1837 and it is here declared nomen oblitum on the basis of art. 23.9 Reversal of precedence established by the ICZN. Sciaena guttata has never been used as a valid name after 1899 (art. 23.9.1.1) whereas Eleotris margaritacea has been used as valid name in genera Ophieleotris or Giuris in at least 25 works published by at least 10 authors encompassing a span of at least 10 years (art. 23.9.1.2). Therefore Eleotris margaritacea Valenciennes, 1837 is here regarded as nomen protectum and according to art. 23.9.1.2 the following 25 works are included: valid as Ophieleotris margaritacea (Valenciennes, 1837) Larson and Murdy (2001); valid as Giuris margaritacea (Valenciennes, 1837), Bauchot et al. (1991), McDowall (1997), Ng et al. (1999), Rees et al. (1999), Allen et al. (2000), Allen et al. (2002), Larson and Pidgeon (2004), Hoese (2006), Boseto et al. (2008), Johnson and Randall (2008), Larson et al. (2008), Fricke et al. (2011), Humphries and Walker (2013), Larson and Sparks (2017), Mutia et al. (2018), Pusey et al. (2017), Sivaperuman and Venkataraman (2018), Sivaperuman et al. (2018), Tsuboi et al. (2018); and valid as Giuris margaritaceus (Valenciennes, 1837) by Kottelat (2013), Miesen et al. (2016), Fricke et al. (2019).

Sciaena lepisma Linnaeus, 1758: 288 (no locality). Described with the following fin formula: D 10/19 P 11; V 1/6; A 3/11; C 13 and diagnosed as "Sciaena pinna dorsali intra 2 folia squamea recondenda". Not a sciaenid, possibly a sparid.

Sciaena pallida Walbaum, 1792: 320 (no locality). No types known.

Comments: Walbaum took the name and part of the description from Linnaeus (1754: 65, pl. 31, fig. 8). Analysis of the original description and illustration reveals that Sciaena pallida is most probably a senior synonym of the labrid Cymolutes praetextatus (Quoy and Gaimard, 1834). Unfortunately, no type locality is reported, however the aim of Linnaeus' book is to describe and illustrate exotic animals. It is inferred that Sciaena pallida came from outside Europe. Description and illustration fit well with C. praetextatus: Br. 5; D. IX,11; P. 12; V. 6; A. II,12; C. 14, truncate. Body pale, elongated and compressed. Head profile steep; canine teeth in front of jaws present; opercle with scales barely visible. Sciaena pallida is here regarded as senior synonym of Cymolutes praetextatus. The application of the art. 23.9 of the Code favors the stability of names declaring Sciaena pallida as nomen oblitum and Cymolutes praetextatus as nomen protectum. According to art. 23.9.1.2 the following 25 works are included: Wass (1984), Victor (1986), Randall et al. (1990), Francis (1993: 165), Francis and Randall (1993), Allen and Smith-Vaniz (1994), Allen (1997), Winterbottom and Anderson (1997), Myers (1999), Fricke (1999), Parenti and Randall (2000), Westneat in Randall and Lim (2000), Hutchins (2001a; b), Gell and Whittington (2002), Allen and Adrim (2003), Garpe and Öhman (2003), Manilo and Bogorodsky (2003), Myers and Donaldson (2003), Randall (2005), Randall et al. (2006), Randall (2007), Fricke et al. (2011), Allen and Erdmann (2012), and Fricke et al. (2018).

Sciaena plumieri Bloch, 1792: 66, Pl. 306 (Martinique Island, West Indies). Spelled plümieri = pluemieri on plate.

Comments: A junior synonym of Conodon nobilis (Linnaeus, 1758), family Haemulidae (Parenti, 2019a).

Sciaena smirnensis Bloch and Schneider, 1801: 82 (Apparently Izmir [Smyrna], Turkey). No types known. 
Comments: Based on Labrus orientalis Hasselquist, 1764: 394 which is well described and clearly recognizable as Pomatomus saltatrix (Linnaeus, 1766), family Pomatomidae. Therefore, Sciaena smirnensis Bloch and Schneider as new synonym is added to the 12 nominal species already placed into synonymy with $P$. saltatrix.

Sciaena unimaculata Linnaeus, 1758, Systema Nat. ed. 10, v. 1: 289 (no locality). It is briefly described as "S. macula fusca in medio utriusque lateris. D. 11/21. P. 1 5. V. 1/6. A. 3/12. C. 1 7 '. The species was later mentioned with additional details in the Museum Adolphi Friderici (Linnaeus, 1764: 81). In Gmelin's edition the Mediterranean Sea is reported as type locality. This is a species of the genus Spicara, but as already stated by Valenciennes (Cuvier and Valenciennes, 1830: 389) the description does not include diagnostic characters to enable identification at the species level.

\section{Unavailable names}

Paraplesichthys Bleeker (ex Kaup) 1876: 335. Masculine. Ancylodon parvipinnis of Gill (= Ancylodon parvipinnis Cuvier, 1830). Type by monotypy. Not available; published in the synonymy of Isopisthus Gill, and apparently never used as valid name or senior homonym. Type as given by Jordan (1919: 383).

Corvina yeddoensis Döderlein in Steindachner and Döderlein, 1883: 35. Not available, name mentioned in passing under Sciaena bleekeri.

Pseudotolithus brunneolus Jordan and Richardson, 1909, Mem. Carnegie Mus., v. 4 (no. 4): 191, Pl. 71 (Kao-Hsiung (Takao), Taiwan). Suppressed (Opinion 1272; see Atrobucca nibe).

Sciaena alba Larrañaga in Devincenzi, 1925, An. Mus. Nac. Hist. Nat. Montev. (Ser. 2), v. 6: 304. Originally as S. timpanistria vel alba [from Uruguay]. Quoted from Larrañaga manuscript, but Devincenzi, 1925 (p. 322, 304) treated as a synonym of Micropogon opercularis (Quoy and Gaimard, 1825); therefore name was not treated as valid and is unavailable (Art. 11d).

\section{Acknowledgements}

The author expresses his gratitude to Philippe Béarez for his comments which help to significantly improve the quality of the manuscript.

\section{References}

Abbott, J. F. (1899). The marine fishes of Peru. Proceedings of the Academy of Natural Sciences of Philadelphia, 51 (2): 324-364.

Agassiz, L. (1848). Nomenclatoris Zoologici Index Universalis, continens nomina systematica classium, ordinum, familiarum et generum animalius omnium, tam viventium quam fossilium, secundum ordinem alphabeticum unicum disposita, adjectis homonymiis plantarum. Jent and Gassmann, Soloduri. viii +393 pp.

Agassiz, L. (1854). Notice of a collection of fishes from the southern bend of the Tennessee River, in the State of Alabama. American Journal of Science and Arts (Series 2), 17: 297-308 (Continued on pp. 353-365).

Aguilera, O. (1983a). Una nueva especie de pez del genero Pachyurus (Perciformes, Sciaenidae) de la region Amazonica de Brasil. Memoria de la Sociedad de Ciencias Naturales La Salle, 43 (119): 119-126. 
Aguilera, O. (1983b). Una nueva especie de pez Stellifer del genero Perciformes, Sciaenidae del noroeste de Venezuela. Memoria de la Sociedad de Ciencias Naturales La Salle, 43 (120): 147-156.

Aguilera, O. A., Schwarzhans, W. and Béarez, P. (2016). Otoliths of the Sciaenidae from the Neogene of tropical America. Palaeo Ichthyologica, 14: 7-90.

Aguilera, O., Solano, O. D. and Valdez, J. (1983). A new species of fish of the genus Stellifer (Perciformes, Sciaenidae) from the South Caribbean Sea. Anales del Instituto de Investigaciones Marinas de Punta de Betín, 13: 5-16.

Alcock, A. W. (1889). Natural history notes from H. M.'s Indian marine survey steamer Investigator,' Commander, Alfred Carpenter, R. N. D. S. O. Commanding. No. 12. Descriptions of some new and rare species of fishes from the Bay of Bengal, obtained during the season of 1888-89. Journal of the Asiatic Society of Bengal, 58 (pt 2, 3): 296305.

Allen, G. R. (1997). Marine fishes of tropical Australia and south-east Asia. Western Australian Museum, Perth. 292 pp.

Allen, G. R. and Adrim, M. (2003). Coral reef fishes of Indonesia. Zoological Studies, 42 (1): $1-72$.

Allen, G. R. and Erdmann, M. V. (2012). Reef fishes of the East Indies. 3 Volumes. Tropical Reef Research, Perth, Australia. vol. III: preface, map, contents and pp. 857-1260; including Appendix 1 (new species descriptions) and Appendix II (addendum).

Allen, G. R. and Robertson, D. R. (1994). Fishes of the tropical eastern Pacific. Crawford House Press, Bathurst. Xx +332 pp.

Allen, G. R. and Smith-Vaniz, W. F. (1994). Fishes of the Cocos (Keeling) Islands. Atoll Research Bulletin, 412: 1-21.

Allen, G. R. and Swainston, R. (1988). The marine fishes of north-western Australia. A field guide for anglers and divers. Western Australian Museum, Perth. vi + 201 pp.

Allen, G. R., Hortle, K. G. and Renyaan, S. J. (2000). Freshwater fishes of the Timika region, New Guinea. PT Freeport Indonesian Company, Timika Environmental Laboratory, Timika. ix + 175 pp.

Allen, G. R., Midgley, S. H. and Allen, M. (2002). Field guide to the freshwater fishes of Australia. Western Australian Museum, Perth, Western Australia. 394 pp.

Araya, H. A. (1984). Los sciaénidos (corvinas) del Golfo de Nicoya, Costa Rica. Revista de Biologia Tropical, 32 (2): 179-196.

Asso y del Rio, I. J. de. (1801). Introduccion á la ichthyologia oriental de España. Anales de Ciencias Naturales, Madrid, 4 (10), 28-52.

Ayres, W. O. (1855). [Description of new fishes from California.] The Pacific [newspaper], 4 (7-22). [Also appeared in Ayres, W. O. (1855) [Descriptions of new species of Californian fishes.] A number of short notices read before the Society at several meetings in 1855. Proceedings of the California Academy of Sciences (Series 1), 1 (pt 1): 23-77.

Ayres, W. O. (1860). [Description of fishes.]. Proceedings of the California Academy of Sciences (Series 1), 2: 77-86.

Ayres, W. O. (1861). [Description of a new ichthyic form from the coast of Lower California.]. Proceedings of the California Academy of Sciences (Series 1), 2: 156-158.

Azpelicueta, M. de las M., Delpiani, S. M., Cione, A. L., Oliveira, C., Marceniuk, A. P. and Díaz de Astarloa, J. M. (2019). Morphology and molecular evidence support the validity of Pogonias courbina (Lacepède, 1803) (Teleostei, Sciaenidae), with a redescription and 
neotype designation. PLoS ONE, $14 \quad$ (6), e0216280, 1-24. https://doi.org/10.1371/journal.pone.0216280

Balletto, E., Cassuolo, L. A. and Bonelli, S. (2014). An annotated Checklist of the Italian Butterflies and Skippers (Papilionoidea, Hesperiioidea). Zootaxa, 3853: 1-114. http://dx.doi.org/10.11646/zootaxa.3853.1.1

Barton, O. (1947). Two new fishes, an Eques and a Holanthias, from Peru. American Museum Novitates, 1350: 1-3.

Basilewsky, S. (1855). Ichthyographia Chinae borealis. Nouveaux mémoires de la Société impériale des naturalistes de Moscou, 10: 215-263.

Bauchot, M. L. and Desoutter, M. (1987). Catalogue critique des types de Poissons du Muséum national d'Histoire naturelle. Famille des Sciaenidae. Bulletin du Museum National d'Histoire Naturelle, Série 4, Section A, Zoologie, Biologie et Écologie Animales, 9 (3): 1-43.

Bauchot, M. L., Desoutter, M., Hoese, D. F. and Larson, H. K. (1991). Catalogue critique des types de Poissons du Muséum national d'Histoire naturelle. (Suite) Sous-ordre des Gobioidei. Bulletin du Museum National d'Histoire Naturelle, Série 4, Section A, Zoologie, Biologie et Écologie Animales, 13 (1-2): 1-82.

Béarez, P. (1996). Lista de los peces marinos del Ecuador continental. Revista de Biologia Tropical, 44 (2): 731-741.

Béarez, P. (2001). Description of a new weakfish, Cynoscion nortoni, from Ecuador with a note on the distribution of Umbrina bussingi (Perciformes, Sciaenidae). Revista de Biología Tropical, 49 (Suppl. 1): 59-65. https://doi.org/10.15517/rbt.v49i1

Béarez, P. and Schwarzhans, W. (2014). Robaloscion, a new genus for Sciaena wieneri Sauvage, 1883 (Teleostei, Sciaenidae) from the southeastern Pacific, with clarification of the status of Sciaena starksi Evermann and Radcliffe, 1917. Cybium, 37 (4): 273-279. https://doi.org/10.26028/cybium/2013-374-006

Ben-Tuvia, A. and Trewavas, E. (1987). Atrobucca geniae, a new species of sciaenid fish from the Gulf of Elat (Gulf of Aqaba), Red Sea. Journal of Zoology, 34 (1-2): 15-21. https://doi.org/10.1080/00212210.1986.10688581

Berg, C. (1895). Enumeración sistemática y sinonímica de los peces de las costas Argentina y Uruguaya. Anales del Museo Nacional de Historia Natural de Buenos Aires, 4: 1-120.

Bleeker, P. (1849a). Bijdrage tot de kennis der ichthyologische fauna van het eiland Madura, met beschrijving van eenige nieuwe soorten. Verhandelingen van het Bataviaasch Genootschap van Kunsten en Wetenschappen, 22 (8): 1-16.

Bleeker, P. (1849b). Bijdrage tot de kennis der Sciaenoïden van den Soenda-Molukschen Archipel, met beschrijving van 7 nieuwe soorten. Verhandelingen van het Bataviaasch Genootschap van Kunsten en Wetenschappen, 23 (5): 1-31.

Bleeker, P. (1851a). Nieuwe bijdrage tot de kennis der ichthyologische fauna van Borneo met beschrijving van eenige nieuwe soorten van zoetwatervisschen. Natuurkundig Tijdschrift voor Nederlandsch Indië, 1 (3): 259-275.

Bleeker, P. (1851b). Derde bijdrage tot de kennis der ichthyologische fauna van Borneo, met beschrijving van eenige nieuwe soorten van zoetwatervisschen. Natuurkundig Tijdschrift voor Nederlandsch Indië, 2 (1): 57-70.

Bleeker, P. (1852). Zesde bijdrage tot de kennis der ichthyologische fauna van Borneo. Visschen van Pamangkat, Bandjermassing, Praboekarta en Sampit. Natuurkundig Tijdschrift voor Nederlandsch Indië, 3 (3): 407-442. 
Bleeker, P. (1853). Diagnostische beschrijvingen van nieuwe of weinig bekende vischsoorten van Sumatra. Tiental V-X. Natuurkundig Tijdschrift voor Nederlandsch Indië, 4 (2): 243-302.

Bleeker, P. (1854a). Nieuwe tientallen diagnostische beschrijvingen van nieuwe of weinig bekende vischsoorten van Sumatra. Natuurkundig Tijdschrift voor Nederlandsch Indië, 5 (3): 495-534.

Bleeker, P. (1854b). Vijfde bijdrage tot de kennis der ichthyologische fauna van Celebes. Natuurkundig Tijdschrift voor Nederlandsch Indië, 7 (2): 225-260.

Bleeker, P. (1855a). Bijdrage tot de kennis der ichthyologische fauna van Batjan. Natuurkundig Tijdschrift voor Nederlandsch Indië, 7 (3): 359-378.

Bleeker, P. (1855b). Achtste bijdrage tot de kennis der ichthyologische fauna van Borneo. Zoetwatervisschen van Bandjermasin. Natuurkundig Tijdschrift voor Nederlandsch Indië, 8 (1): 151-168.

Bleeker, P. (1855c). Zesde bijdrage tot de kennis der ichthyologische fauna van Amboina. Natuurkundig Tijdschrift voor Nederlandsch Indië, 8 (3): 391-434.

Bleeker, P. (1858). Zevende bijdrage tot de kennis der vischfauna van Sumatra. Visschen van Palembang. Acta Societatis Regiae Scientiarum Indo-Neêrlandicae, 5 (art. 6): 1-12.

Bleeker, P. (1863a). Description de quelques espèces de poissons, nouvelles ou peu connues de Chine, envoyées au Musée de Leide par M.-G. Schlegel. Nederlandsch Tijdschrift voor de Dierkunde, 1: 135-150.

Bleeker, P. (1863b). Mémoire sur les poissons de la côte de Guinée. Natuurkundige Verhandelingen van de Hollandsche Maatschappij der Wetenschappen te Haarlem (Ser. 2), 18: 1-136.

Bleeker, P. (1873). Description (avec figures) de deux espèces nouvelles de Sciénoïdes de Surinam. Archives néerlandaises des sciences exactes et naturelles, 8: 456-461, Pl. 21.

Bleeker, P. (1874). Mémoire sur les Sciénoïdes et les Sillaginoïdes de l'Inde archipélagique. Verhandelingen der Koninklijke Akademie van Wetenschappen, Afdeeling Natuurkunde (Amsterdam), 14 (art. 4): 1-76.

Bleeker, P. (1876). Systema Percarum revisum. Pars II. Archives néerlandaises des sciences exactes et naturelles, 11: 289-340.

Bleeker, P. (1877). [Over slokdarm, maag en dunnen darm van Caprodon Schlegeli. De Heer Bleeker ... Hamburger Museum ... visschen van China.]. Processen-verbaal van de gewone vergaderingen der Koninklijke Akademie van Wetenschappen. Afdeeling Natuurkunde 1877-1878, (5): 2-3.

Bleeker, P. (1879). Sur quelques espèces inédites ou peu connues de poissons de Chine appartenant au Muséum de Hambourg. Verhandelingen der Koninklijke Akademie van Wetenschappen, Afdeeling Natuurkunde (Amsterdam), 18: 1-17.

Bloch, M. E. (1788). Beskrivelse over tvende nye Aborrer fra Indien. KongeligeDanskeVidenskabernes Selskabs Skrivter, Nye Samling af det, 3: 383-385.

Bloch, M. E. (1790). Naturgeschichte der ausländischen Fische. Mit sechs und dreissig ausgemalten kupfern nach originalen. Vierter Theil. Auf Kosten des Verfassers und in Commission bei dem Buchhändler Hr. Hesse, Berlin. xii + 128 pp.

Bloch, M. E. (1792). Naturgeschichte der ausländischen Fische. Mit sechs und dreissig ausgemalten kupfern nach originalen. Sechster Theil. Auf Kosten des Verfassers und in Commission bei dem Buchhändler Hr. Hesse, Berlin. xii + 126 pp. 
Bloch, M. E. (1793). Naturgeschichte der ausländischen Fische. Mit sechs und dreissig ausgemalten kupfern nach originalen. Siebenter Theil. Auf Kosten des Verfassers und in Commission bei dem Buchhändler Hr. Hesse, Berlin. xiv + 144 pp.

Bloch, M. E. (1797). Ichthyologie, ou Histoire naturelle, générale et particulière des poissons. Avec des figures enluminées dessinées d'apres nature. Dixième partie. Avec 36 planches. Berlin chez l'Auteur. v + 125 pp.

Bloch, M. E. and Schneider, J. G. (1801). M. E. Blochii, Systema Ichthyologiae Iconibus cx Ilustratum. Post obitum auctoris opus inchoatum absolvit, correxit, interpolavit Jo. Gottlob Schneider. Sumtibus Auctoris Impressum et Bibliopolio Sanderiano Commissum, Berolini. lx +584 pp.

Blyth, E. (1860). Report on some fishes received chiefly from the Sitang River and its tributary streams, Tenasserim Provinces. Journal of the Asiatic Society of Bengal, 29 (2): $138-174$.

Bocourt, F. (1869). Descriptions de quelques reptiles et poissons nouveaux appartenant à la faune tropicale de l'Amérique. Bulletin des Nouvelles Archives du Muséum d'Histoire Naturelle, Paris, 5: 19-24.

Boeseman, M. (1947). Revision of the fishes collected by Burger and Von Siebold in Japan. Zoologische Mededelingen (Leiden), 28: i-vii + 1-242.

Boeseman, M. (1948). On new, little known, or otherwise interesting species of Surinam fishes. Proceedings of the Section of Sciences, Koninklijke Nederlandse Akademie van Wetenschappen, 51 (3): 363-371.

Boeseman, M. (1963). An annotated list of fishes from the Niger Delta. Zoologische Verhandelingen (Leiden), 61: 1-48.

Böhlke, E. B. (1953). A catalogue of the type specimens of Recent fishes in the Natural History Museum of Stanford University. Stanford Ichthyological Bulletin, 5 (1): 1-168.

Böhlke, E. B. (1984). Catalog of the type specimens in the ichthyological collection of the Academy of Natural Sciences of Philadelphia. Special Publication, Academy of Natural Sciences of Philadelphia, 14: 246 pp.

Bonaparte, C. L. (1831). Prospetto del sistema generale d'Ittiologia. Giornale Arcadico di Scienze Lettere ed Arti, 52: 155-189.

Borodin, N. A. (1930). Scientific results of the Yacht "Ara" Expedition during the years 1926 to 1930, while in command of William K. Vanderbilt. Fishes (collected in 1929). Bulletin Vanderbilt Marine Museum, 1 (2): 39-64.

Borodin, N. A. (1932). Fishes. Scientific results of the yacht "Alva" world cruise, July, 1931 to March, 1932, in command of William K. Vanderbilt. Bulletin Vanderbilt Marine Museum, 1 (3): 65-101.

Boschung, H. T. (1992). Catalogue of freshwater and marine fishes of Alabama. Bulletin of the Alabama Museum Natural History, 14: i-xvi + 1-266.

Boseto D., Morrison, C., Pikacha P. and Pitakia, T. (2008). Biodiversity and conservation of freshwater fishes in selected rivers on Choiseul Island, Solomon Islands. The South Pacific Journal of Natural Science, 25 (1): 16-21.

Boulenger, G. A. (1888). An account of the fishes obtained by Surgeon-Major A. S. G. Jayakar at Muscat, east coast of Arabia. Proceedings of the Zoological Society of London, 1887 (pt 4): 653-667.

Boulenger, G. A. (1895). [Account of a large collection of fishes formed by Dr. C. Ternetz at various localities in Matto Grosso and Paraguay.]. Proceedings of the Zoological Society of London, 1895 (pt 3): 523-529. 
Boulenger, G. A. (1899). Viaggio del Dr. Enrico Festa nell'Ecuador e regioni vicine. Poissons de l'Équateur. [Deuxième Partie] (1). Bollettino dei Musei di Zoologia ed Anatomia Comparata della R. Università di Torino, 14 (335): 1-8.

Bowdich, S. L. (1825). Fishes of Madeira, In: Bowdich, T. E. (Ed.), Excursions in Madeira and Porto Santo during the autumn of 1823, while on his third voyage to Africa. George B. Whittaker, London. pp. 121-125 and 233-238.

Breder, C. M. and Rosen, D. E. (1966). Modes of reproduction in fishes. T. F. H. Publications, Neptune City, New Jersey. 941 pp.

Browne, P. (1756). The civil and natural history of Jamaica. In three parts. T. Osborne and J. Shipton, London. viii $+490 \mathrm{pp}$.

Bussing, W. A. and López, S. M. I. (1994). Demersal and pelagic inshore fishes of the Pacific coast of lower Central America. An illustrated guide. Revista de Biología Tropical Special Publication, 1: 1-164.

Cadenat, J. (1951). Initiations Africaines. III. Poissons de mer du Sénégal. Institute Francais d'Afrique Noire, Dakar. 345 pp.

Caldwell, D. K. (1958). A new fish of the genus Ophioscion, family Sciaenidae, from Caribbean Costa Rica. Quarterly Journal of the Florida Academy of Sciences, 21 (2): 117-124.

Cantor, T. E. (1849). Catalogue of Malayan fishes. Journal of the Asiatic Society of Bengal, 18 (pt 2): i-xii + 983-1443.

Carpenter, K. E., Krupp, F., Jones, D. A. and Zajonz, U. (1997). FAO species identification guide for fishery purposes. The living marine resources of Kuwait, eastern Saudi Arabia, Bahrain, Qatar, and the United Arab Emirates. FAO, Rome. 293 pp.

Carvalho-Filho, A., Santos, S. and Sampaio, I. (2010). Macrodon atricauda (Günther, 1880) (Perciformes: Sciaenidae), a valid species from the southwestern Atlantic, with comments on its conservation. Zootaxa, 2519: 48-58. https://doi.org/10.5281/zenodo.196207

Casatti, L. (2001). Taxonomia do gênero Sul-Americano Pachyurus Agassiz, 1831 (Teleostei, Perciformes, Sciaenidae) e descrição de duas novas espécies. Comunicações do Museu de Ciências da PUCRS, 14 (2): 133-178.

Casatti, L. (2002a). Taxonomy of the South American genus Pachypops Gill 1861 (Teleostei, Perciformes, Sciaenidae), with the description of a new species. Zootaxa, 26 (1): 1-20. http://dx.doi.org/10.11646/zootaxa.26.1.1

Casatti, L. (2002b). Petilipinnis, a new genus for Corvina grunniens Schomburgk, 1843 (Perciformes, Sciaenidae) from the Amazon and Essequibo river basins and redescription of Petilipinnis grunniens. Papéis Avulsos de Zoologia, Museu de Zoologia da Universidade de São Paulo, 42 (7): 169-181.

Casatti, L. (2005). Revision of the South American freshwater genus Plagioscion (Teleostei, Perciformes, Sciaenidae). Zootaxa, 1080 (1): 39-64. http://dx.doi.org/10.11646/zootaxa. 1080.1.4

Casatti, L. and Chao, L. N. (2002). A new species of Pachyurus Agassiz 1831 (Teleostei, Perciformes, Sciaenidae) from the Río Napo basin, eastern Ecuador. Zootaxa, 38 (1): 17. http://dx.doi.org/10.11646/zootaxa.38.1.1

Castelnau, F. L. (1855). Animaux nouveaux or rares recueillis pendant l'expédition dans les parties centrales de l'Amérique du Sud, de Rio de Janeiro a Lima, et de Lima au Para; exécutée par ordre du gouvernement Français pendant les années 1843 a 1847 sous la 
direction du Compte Francis de Castelnau. Tome second. Poissons. P. Bertrand, Paris. xii +112 pp.

Castelnau, F. L. (1861). Mémoire sur les poissons de l'Afrique australe. J.-B. Baillière et Fils, Paris. vii +78 pp.

Castelnau, F. L. (1872). Contribution to the ichthyology of Australia. No. 1.The Melbourne fish market. No. II. Note on some South Australian fishes. Proceedings of the Zoological and Acclimatisation Society of Victoria, Melbourne, 1: 29-247.

Castro-Aguirre, J. L. (1978). Catalogo sistematico de los peces marinos que penetran a las aguas continentales de Mexico con aspectos zoogeograficos y ecologicos. Serie cientifica 19. Departamento de Pesca, Dirección General del Instituto Nacional de Pesca, México City. 298 pp.

Castro-Aguirre, J. L. and Arvizu-Martínez, J. (1976). Una nueva especie de Cynoscion, del Pacifico de México (Pisces, Sciaenidae, Otholitinae). Revista de la Sociedad Mexicana de Historia Natural, 37: 323-329.

Castro-Aguirre, J. L., Espinosa Pérez, H. and Schmitter-Soto, J. J. (1999). Ictiofauna estuarino-lagunar y vicaria de México. Noriega-Limusa, IPN, Mexico City. 711 pp.

Cervigón, F. (1966). Una nueva especie de Stellifer de las costas de Venezuela (Pisces: Sciaenidae). Novedades Cientificas, Contribuciones Ocasionales del Museo de Historia Natural La Salle, Serie Zoológica, 34: 1-4.

Cervigón, F. (1982). La ictiofauna estuarina del Caño Manamo y areas adyacentes, In: Novoa, D. (Ed.), Los recursos pesqueros del Rio Orinoco y su explotacion. Corporación Venezolana de Guayana, Caracas. pp. 205-260.

Cervigón, F. (2011). Los peces marinos de Venezuela. Fundación Científica Los Roques, Caracas, Venezuela. $130 \mathrm{pp}$.

Cervigón, F., Cipriani, R., Fischer, W., Garibaldi, L., Hendrickx, M., Lemus, A. J., Márquez, R., Poutiers, J. M., Robaina, G. and Rodriguez, B. (1993). Field guide to the commercial marine and brackish-water resources of the northern coast of South America. FAO Species Identification Sheets for Fishery Purposes. FAO, Rome. 513 pp.

Chabanaud, P. (1926). Description d'un poisson nouveau d'Indochine, appartenant à la famille des Sciaenidae. Bulletin du Muséum National d'Histoire Naturelle (Série 1), 32 (5): 266270.

Chao, L. N. (1978). A basis for classifying western Atlantic Sciaenidae (Teleostei, Perciformes). NOAA Technical Report NMFS Circular 415, 1-64 pp.

Chao, L. N. (1986). A synopsis on zoogeography of the Sciaenidae, In: Uyeno, T., Arai, R., Taniuchi, T., and Matsuura, K. (Eds.), Indo-Pacific fish biology, Proceedingsof the Second International Conference of the Indo-Pacific Fishes, July-August 1985. Ichthyological Society of Japan, Tokyo. pp. 570-589.

Chao, L. N. (1995). Sciaenidae, In: Fischer, W., Krupp, F., Schneider, W., Sommer, C., Carpenter, K. E. and Niem, V. (Eds.), Guia FAO para identificacion de especies para los fines de la pesca. Pacifico Centro-oriental. FAO, Rome. pp. 1427-1518.

Chao, L. N. (2001). Two new species of Stellifer from inshore waters of the eastern Pacific, with a redescription of $S$. ephelis (Perciformes, Sciaenidae). Revista de Biología Tropical, 49 (Suppl. 1): 67-80.

Chao, L. N. (2002a). Taxonomy of the seatrout, genus Cynoscion (Pisces, Sciaenidae), with artificial keys to the species, In: Bortone, S. A. (Ed.), Biology of the spotted seatrout. CRC Marine Biology Series, Taylor and Francis Group, Boca Raton, Florida. pp. 5-15. 
Chao, L. N. (2002b). Sciaenidae, In: Carpenter, K. E. (Ed.), The living marine resources of the Western Central Atlantic. Volume 3: Bony fishes part 2 (Opistognathidae to Molidae), sea turtles and marine mammals. FAO Species Identification Guide for Fishery Purposes and American Society of Ichthyologists and Herpetologists Special Publication No. 5. FAO, Rome. pp. 1583-1653.

Chao, L. N. (2016). Sciaenidae, In: Carpenter, K. E. and De Angelis, N. (Eds.), The living marine resources of the Eastern Central Atlantic. Volume 4. Bony fishes part 2 (Perciformes to Tetraodontiformes) and Sea turtles. FAO Species Identification Guide for Fishery Purposes, FAO, Rome. pp. 2629-2654.

Chao, L. N. and Miller, R. V. (1975). Two new species of sciaenid fishes (Tribe, Sciaenini) from the Caribbean Sea and adjacent waters. Bulletin of marine Science, 25 (2): 259271.

Chao, L. N. and Trewavas, E. (1990). Sciaenidae, In: Quero, J. C., Hureau, J. C., Karrer, C., Post, A. and Saldanha, L. (Eds.), Checklist of the fishes of the eastern Tropical Atlantic (CLOFETA). Unesco, Paris. pp. 813-826.

Chao, L. N., Béarez, P. and Robertson, D. R. (2001). A new genus and new species of Sciaenidae from the Gulf of Panama (Perciformes, Sciaenidae). Revista de Biología Tropical, 49 (Suppl. 1): 81-88.

Chao, L. N., Chang, C. W., Chen, M. H., Guo, C. C., Lin, B. A., Liou, Y. Y., Shen, K. N. and Liu, M. (2019). Johnius taiwanensis, a new species of Sciaenidae from the Taiwan Strait, with a key to Johnius species from Chinese waters. Zootaxa, 4651 (2): 259-270. http://dx.doi.org/10.11646/zootaxa.4651.2.3

Chen, Q. C., Cai, Y. Z. and Ma, X. M. (1997). Fishes from Nansha Islands to South China Coastal Waters 1. Science Press. i-xx + 1-202 pp.

Chirichigno, F. N. (1962). Algunos peces nuevos y poco conocidos de la fauna marina del Perú. Serie de Divulgación Cientifica, Servicio de Pesquería, Ministerio de Agricultura, Lima, 17: 1-29.

Chirichigno, F. N. (1969). Ctenosciaena peruviana nov. sp. Una nueva especie de Sciaenidae de la costa del Perú. Informes Especiales, Instituto del Mar del Perú (IMARPE). Callao. No. 48, 18 unnumbered pp.

Chirichigno, F. N. (1974). Clave para identificar los peces marinos del Peru. Instituto del Mar del Perú, Callao. $387+2$ pp.

Chirichigno, F. N. and Vélez, D. J. (1998). Clave para identificar los peces marinos del Peru (Seguenda edición, revidada y actualizada). Instituto del Mar del Peru, Multiformas, Callao, Peru. 496 pp.

Chu, Y. T., Lo, Y. L. and Wu, H. L. (1963). Monographs of fishes of China. A study on the classification of the sciaenoid fishes of China, with description of new genera and species. The Collective Papers of Science and Technology Research of Shanghai City, Shanghai. $100 \mathrm{pp}$.

Cobo, M. and Massay, S. (1969). Lista de peces marinos del Ecuador. Boletin Cientifico Téchnico Instituto Nacional de Pesca, 2: 1-68.

Cope, E. D. (1867). Supplement on some new species of American and African fishes. Transaction American Philosophical Society, 13: 400-407.

Corpuz, M. N. C., Paller, V. G. V. and Ocampo, P. P. (2015). Environmental variables structuring the stream gobioid assemblages in the three protected areas in Southern Luzon, Philippines. The Raffles Bulletin of Zoology, 63: 357-365. 
Costa, F. J. S., Coutinho, D. P. and Wosiacki, W. B. (2019). Phylogenetic relationships of the species of Plagioscion Gill, 1861 (Eupercaria, Sciaenidae). Zoology (Jena), 132: 41-56. http://dx.doi: 10.1016/j.zool.2019.01.001

Cousseau, M. B. and Perrotta, R. G. (2000). Peces marinos de Argentina. Biologia, distribución, pesca. Instituto Nacional de Investigación y Desarrollo Pesquero (INIDEP), Mar del Plata (Argentina). 168 pp.

Cuvier, G. (1816a). Dictionnaire des sciences naturelles, dans lequel on traite méthodiquement des différens êtres de la nature, considérés soit en eux-mêmes, d'après l'état actuel de nos connoissances, soit relativement à l'utilité qu'en peuvent retirer la médecine, l'agriculture, le commerce et les artes. Suivi d'une biographie des plus célèbres naturalistes ... Tome Second. F. G. Levrault, Strasbourg, et Le Normant, Paris, 515 pp. +123 pp. Supplement.

Cuvier, G. (1816b). Le Règne Animal distribué d'après son organisation pour servir de base à l'histoire naturelle des animaux et d'introduction à l'anatomie comparée. Les reptiles, les poissons, les mollusques et les annélides. Tome II. A. Belin, Paris. xviii +532 pp.

Cuvier, G. (1829). Le Règne Animal, distribué d'après son organisation, pour servir de base à l'histoire naturelle des animaux et d'introduction à l'anatomie comparée. Nouvelle Édition, Revue et Augmentée. Tome II. Déterville et Crochard, Paris. xv + 406 pp.

Cuvier, G. and Valenciennes, A. (1830). Histoire naturelle des poissons. Tome cinquième. F.G. Levrault, Paris. xxviii $+499+4$ pp.

Cuvier, G. and Valenciennes, A. (1833). Histoire naturelle des poissons. Tome neuvième. F. G. Levrault, Paris. xxix $+3+512$ pp.

Daget, J. and Trewavas, E. (1986). Sciaenidae, In: Daget, J., Gosse, J. P. and Thys van den Audenaerde, D. F. E. (Eds.), Check-list of the freshwater fishes of Africa. CLOFFA. ISNB Bruxelles, MRAC Tervuren, ORSTOM Paris. Volume 2, pp. 333-337.

Daneri, C. A. (1954). Una nueva especie de "Corvina de agua dulce" Plagioscion macdonaghi n. sp. (Pisces, Sciaenidae). Comunicaciones del Museo de Ciencas Naturales "Bernardino Rivadavia" e Instituto Nacional de Investigación de las Ciencias Naturales, Ciencias Zoológicas, 2 (12): 179-188.

Daneri, C. A. (1956). Pachyurus paranensis n. sp. de corvina de agua dulce (Pisces, Sciaenidae). Comunicaciones Jornadas Icticas, Santa Fe, Dirección Principal de Biología Animal y Zootecnía, 1956: 1-16.

Day, F. (1873). On some new fishes of India. The Journal of the Linnean Society of London. Zoology, 11: 524-530.

Day, F. (1876). The fishes of India; being a natural history of the fishes known to inhabit the seas and fresh waters of India, Burma, and Ceylon. Bernard Quaritch, London. 320 pp + xii (index).

de Bruin, G. H. P., Russell, B. C. and Bogusch, A. (1995). The marine fishery resources of Sri Lanka. FAO Species Identification Field Guide for Fishery Purposes. FAO, Rome. 400 pp.

de Buen, F. (1961). Peces Chilenos. Familias Alepocephalidae, Muraenidae, Sciaenidae, Scorpaenidae, Liparidae y Bothidae. Montemar, 1: 1-52.

de la Pylaie, A. J. M. (1835). Recherches, en France, sur les poissons de l'Océan, pendant les années 1832 et 1833. Congrès Scientifique de France, Poitiers. (art. 5): 524-534.

de Vis, C. W. 1884). New fishes in the Queensland Museum. No. 3. Proceedings of the Linnean Society of New South Wales, 9 (pt 3): 537-547. 
Delfin, F. T. (1900). Nota del Ictiolojia. El nuevo jénero Cilus. Actes de la Société Scientifique du Chili, 10: 53-60.

Delsman, H. C. (1941). Résultats scientifiques des croisières du Navire-école Belge "Mercator", vol. III, No. 3. Pisces. Mémoires du Musée royal d'histoire naturelle de Belgique (Ser. 2), 21: 47-82.

Desmarest, A. G. (1823). Première Décade Ichthyologique, ou, Description complète de dix espèces de poissons nouvelles, ou imparfaitement connues, habitant la mer qui baigne les côtes de l'ile de Cuba. Mémoires de la Société linnéenne de Paris, 2: 163-210.

Devincenzi, G. J. (1925). El primer ensayo sobre ictiología del Uruguay. La clase "Peces" de la zoología de Don Dámaso A. Larrañaga. Anales del Museo Nacional de Historia Natural de Montevideo (Serie 2), 1 (pt. 6): 295-323.

Edwards, G. (1751). A natural history of Birds...containing The Reproductions of thirty-nine of Birds,...To which are added, by way of Appendix, Sixteen Copper-Plates, representing the Figures of many curious and undescribed Animals, such as Quadrupedes (both Land and Amphibious) Serpents, Fishes and Insects, The whole Containing Fifty-thee CopperPlates,... Part IV and Last. Printed for the Author, at the College of Physicians, London. $248 \mathrm{pp}$.

Ehrenbaum, E. (1915). Über Küstenfische von Westafrika, besonders von Kamerun. L. Friederichsen and Co., Hamburg. 85 pp.

Eigenmann, C. H. (1918). Eighteen new species of fishes from northwestern South America. Proceedings of the American Philosophical Society, 56 (7): 673-689.

Evermann, B. W. and Radcliffe, L. (1917). The fishes of the west coast of Peru and the Titicaca Basin. Bulletin of the United States National Museum, 95: i-xi + 1-166.

Eydoux, J. F. T. and Souleyet, F. A. (1850). Poissons, In: Voyage autour du monde exécuté pendant les années 1836 et 1837 sur la corvette La Bonite, commandée par M. Vaillant Capitaine de Vaisseau. Zoologie, Tome Premier, seconde partie. Arthus Bertrand, Paris. pp. 155-216.

Figueiredo, J. L. de (1992). Sobre a applicação dos nomes Cynoscion striatus (Cuvier, 1829) eCynoscion guatucupa (Cuvier, 1830). Teleostei, Sciaenidae. Comunicações do Museu de Ciências da Pontifícia Universidade Católica do Rio Grande do Sul, 5 (8): 117-121.

Fowler, H. W. (1916). Cold-blooded vertebrates from Costa Rica and the Canal Zone. Proceedings of the Academy of Natural Sciences of Philadelphia, 68: 389-414.

Fowler, H. W. (1919). Notes on tropical American fishes. Proceedings of the Academy of Natural Sciences of Philadelphia, 71: 128-155.

Fowler, H. W. (1925). New taxonomic names of West African marine fishes. American Museum Novitates, 162: 1-5.

Fowler, H. W. (1926). Notes on fishes from Bombay. Journal of the Bombay Natural History Society, 31 (pt 3): 770-779.

Fowler, H. W. (1931). A small collection of fishes from Singapore. Proceedings of the Academy of Natural Sciences of Philadelphia, 83: 443-448.

Fowler, H. W. (1933). Contributions to the biology of the Philippine Archipelago and adjacent regions. The fishes of the families Banjosidae...Enoplosidae collected by the United States Bureau of Fisheries steamer "Albatross," chiefly in Philippine seas and adjacent waters. Bulletin of the United States National Museum, 100 (12): i-vi + 1-465.

Fowler, H. W. (1934). Zoological results of the third De Schauensee Siamese Expedition, Part I. - Fishes. Proceedings of the Academy of Natural Sciences of Philadelphia, 86: 67-163. 
Fowler, H. W. (1935). Zoological results of the Third de-Schauensee Siamese Expedition, Part VI - Fishes obtained in 1934. Proceedings of the Academy of Natural Sciences of Philadelphia, 87: 89-163.

Fowler, H. W. (1937). A collection of Haitian fishes obtained by Mr. Stanley Woodward. Proceedings of the Academy of Natural Sciences of Philadelphia, 89: 309-315.

Fowler, H. W. (1944). Results of the fifth George Vanderbilt expedition (1941). (Bahamas, Caribbean Sea, Panama, Galápagos Archipelago and Mexican Pacific islands). The Fishes. Monographs of the Academy of Natural Sciences of Philadelphia, 6: 57-529.

Fowler, H. W. (1954). Os peixes de agua doce do Brasil. Arquivos de Zoologia do Estado Sao Paulo, 9: 1-400.

Fowler, H. W. and Bean, B. A. (1923). Descriptions of eighteen new species of fishes from the Wilkes Exploring Expedition, preserved in the United States National Museum. Proceedings of the United States National Museum, 63 (2488) (art. 19): 1-27.

Francis, M. P. (1993). Checklist of the coastal fishes of Lord Howe, Norfolk, and Kermadec Islands, Southwest Pacific Ocean. Pacific Science, 47 (2): 136-170. http://dx.doi.org/10.6084/m9. figshare.c.4428305

Francis, M. P. and Randall, J. E. (1993). Further additions to the fish faunas of Lord Howe and Norfolk Islands, Southwest Pacific Ocean. Pacific Science, 47 (2): 118-135.

Fricke R. (1999). Fishes of the Mascarene Islands (Réunion, Mauritius, Rodriguez). An annotated checklist with descriptions of new species. Theses Zoologicae v. 31. Koeltz Scientific Books, Koenigstein, Germany. viii + 759 pp.

Fricke, R., Allen, G. R., Amon, D., Andréfouët, S., Chen, W. J., Kinch, J., Mana, R., Russell, B. C., Tully, D. and White, W. T. (2019). Checklist of the marine and estuarine fishes of New Ireland Province, Papua New Guinea, western Pacific Ocean, with 810 new records. Zootaxa, 4588 (1): 1-360. http://dx.doi.org/10.11646/zootaxa.4588.1.1

Fricke, R., Eschmeyer, W. N. and van der Laan, R. (2019). Eschmeyer's Catalog of Fishes: Genera, Species, References. Electronic version. http://researcharchive.calacademy.org (Accessed 16 November 2019).

Fricke, R., Kulbicki, M. and Wantiez, L. (2011). Checklist of the fishes of New Caledonia, and their distribution in the Southwest Pacific Ocean (Pisces). Stuttgarter Beiträge zur Naturkunde A, Neue Serie, 4: 341-463.

Fricke, R., Mahafina, J., Behivoke, F., Jaonalison, H., Léopold, M. and Ponton, D. (2018). Annotated checklist of the fishes of Madagascar, southwestern Indian Ocean, with 158 new records. FishTaxa, 3 (1): 1-432.

Furnestin, J., Dardignac, J., Maurin, C., Vincent, A., Coupé, R. and Boutière, H. (1958). Données nouvelles sur les poissons du Maroc atlantique. Revue des Travaux de l'Institut des Pêches Maritimes, 22 (4): 381-493.

Garpe, K. C. and Öhman, M. C. (2003). Coral and fish distribution patterns in Mafia Island Marine Park, Tanzania: fish-habitat interactions. Hydrobiologia, 498 (1-3): 191-211. https://doi.org/10.1023/A: 1026217201408

Gell, F. R. and Whittington, M. W. (2002). Diversity of fishes in seagrass beds in the Quirimba Archipelago, northern Mozambique. Marine and Freshwaters Research, 53 (2): 115-121. https://doi.org/10.1071/MF01125

Geoffroy St. Hilaire, E. (1809). Histoire Naturelle des poissons du Nil, In: Commission des sciences et arts d'Egypte, Descriptionde l'Égypte, ou, Recueil des observations et des recherches qui ont été faites en Égypte pendant l'expédition de l'armée française, publié 
par les ordres de sa Majesté l'Empereur Napoléon Le Grand. Histoire naturelle. Tome Premier. Imprimerie Impériale, Paris. pp. 1-52.

Gilbert, C. H. (1890). A preliminary report on the fishes collected by the steamer Albatross on the Pacific coast of North America during the year 1889, with descriptions of twelve new genera and ninety-two new species. Proceedings of the United States National Museum, 13 (797): 49-126.

Gilbert, C. H. (1897). Descriptions of twenty-two new species of fishes collected by the steamer Albatross, of the United States Fish Commission. Proceedings of the United States National Museum, 19 (1115): 437-457.

Gilbert, C. H. and Starks, E. C. (1904). The fishes of Panama Bay. Memoirs of the California Academy of Sciences, 4: 1-304.

Gilbert, C. R. (1966). Western Atlantic Sciaenid fishes of the genus Umbrina. Bulletin of marine Science, 16 (2): 230-258.

Gilchrist, J. D. F. and Thompson, W. W. (1908). Descriptions of fishes from the coast of Natal. Annals of the South African Museum, 6 (pt 2): 145-206.

Gilchrist, J. D. F. and Thompson, W. W. (1911). Descriptions of fishes from the coast of Natal (Part III.). Annals of the South African Museum, 11 (pt 2): 29-58.

Gill, T. N. (1861a). Catalogue of the fishes of the eastern coast of North America, from Greenland to Georgia. Proceedings of the Academy of Natural Sciences of Philadelphia, 13 (Suppl.): 1-63.

Gill, T. N. (1861b). On several new generic types of fishes contained in the museum of the Smithsonian Institution. Proceedings of the Academy of Natural Sciences of Philadelphia, 13: 77-78.

Gill, T. N. (1861c). Revision of the genera of North American Sciaeninae. Proceedings of the Academy of Natural Sciences of Philadelphia, 13: 79-89.

Gill, T. N. (1861d). On the Haploidonotinae. Proceedings of the Academy of Natural Sciences of Philadelphia, 13: 100-105.

Gill, T. N. (1862a). Note on the sciaenoids of California. Proceedings of the Academy of Natural Sciences of Philadelphia, 14: 16-18.

Gill, T. N. (1862b). Catalogue of the fishes of Lower California, in the Smithsonian Institution, collected by Mr. J. Xantus. Part III. Proceedings of the Academy of Natural Sciences of Philadelphia, 14: 249-262.

Gill, T. N. (1863a).Catalogue of the North American sciaenoid fishes. Proceedings of the Academy of Natural Sciences of Philadelphia, 15: 28-32.

Gill, T. N. (1863b). Descriptive enumeration of a collection of fishes from the western coast of Central America, presented to the Smithsonian Institution by Captain John M. Dow. Proceedings of the Academy of Natural Sciences of Philadelphia, 15: 162-174.

Gill, T. N. (1903). On some fish genera of the first edition of Cuvier's Règne Animal and Oken's names. Proceedings of the United States National Museum, 26 (1346): 965-967.

Ginsburg, I. (1930). Review of the weakfishes (Cynoscion) of the Atlantic and Gulf coasts of the United States, with a description of a new species. Bulletin of the Bureau of Fisheries, 45: 71-85.

Ginsburg, I. (1952). Eight new fishes from the Gulf coast of the United States, with two new genera and notes on geographic distribution. Journal of the Washington Academy of Sciences, 42 (3): 84-101. 
Girard, C. F. (1854). Observations upon a collection of fishes made on the Pacific coast of the United States, by Lieut. W. P. Trowbridge, U.S.A., for the museum of the Smithsonian Institution. Proceedings of the Academy of Natural Sciences of Philadelphia, 7: 142-156.

Girard, C. F. (1858a). Fishes. In: Reports of explorations and surveys, to ascertain the most practicable and economical route for a railroad from the Mississippi River to the Pacific Ocean. Made under the directory of the Secretariat of War, in 1853-6, according to acts of Congress of March 3, 1853, May 31, 1854, and August 5, 1854. Volume 10, pt. 4. Beverley Tucker, Washington, D. C., xiv + 400 pp.

Girard, C. F. (1858b). Notes upon various new genera and new species of fishes, in the museum of the Smithsonian Institution, and collected in connection with the United States and Mexican boundary survey: Major William Emory, Commissioner. Proceedings of the Academy of Natural Sciences of Philadelphia, 10: 167-171.

Gistel, J. (1848). Naturgeschichte des Thierreichs, für höhere Schulen. Hoffmannsche Verlags-Buchhandlung, Stuttgart. xvi + $216 \mathrm{pp}$.

Goode, G. B. (1876). Catalogue of the fishes of the Bermudas. Based chiefly upon the collection of the United States National Museum. Bulletin of the United States National Museum, 1 (5): 1-82.

Gray, J. E. (1854). Catalogue of fish collected and described by Laurence Theodore Gronow, now in the British Museum. Woodfall and Kinder, London. vii + 196 pp.

Griffiths, M. H. and Heemstra, P. C. (1995). A contribution to the taxonomy of the marine fish genus Argyrosomus (Perciformes, Sciaenidae), with descriptions of two new species from Southern Africa. Ichthyological Bulletin of the J. L. B. Smith Institute of Ichthyology, 65: 1-40.

Günther, A. (1860). Catalogue of the acanthopterygian fishes in the collection of the British Museum. Second Volume. Squamipinnes, Cirrhitidae, Triglidae, Trachinidae, Sciaenidae, Polynemidae, Sphyraenidae, Trichiuridae, Scombridae, Carangidae, Xiphiidae. Printed by Order of the Trustees, London. xxi $+548 \mathrm{pp}$.

Günther, A. (1864). Report of a collection of fishes made by Messrs. Dow, Godman, and Salvin in Guatemala. Part first. Proceedings of the Zoological Society of London, 1864 (pt 1) (art. 3): 144-154.

Günther, A. (1865). Description of a new species of Corvina from the Gambia. Annals and Magazine of Natural History (Series 3), 16 (91) (art. 7): 48-49.

Günther, A. (1867a). On the fishes of the states of Central America, founded upon specimens collected in fresh and marine waters of various parts of that country by Messrs. Salvin and Godman and Capt. J. M. Dow. Proceedings of the Zoological Society of London, 1866 (pt 3): 600-604.

Günther, A. (1867b). Additions to the knowledge of Australian reptiles and fishes. Annals and Magazine of Natural History (Series 3), 20 (115): 45-68.

Günther, A. (1868). An account of the fishes of the states of Central America, based on collections made by Capt. J. M. Dow, F. Godman, Esq., and O. Salvin, Esq. Transactions of the Zoological Society of London, 6 (14): 377-494.

Günther, A. (1872). Notice of some species of fishes from the Philippine Islands. Annals and Magazine of Natural History (Series 4), 10 (59) (art. 56): 397-399.

Günther, A. (1873). Erster ichthyologischer Beitrag nach Exemplaren aus dem Museum Godeffroy. Journal des Museum Godeffroy, 1 (2): 97-103.

Günther, A. (1874). Descriptions of new species of fishes in the British Museum. Annals and Magazine of Natural History (Series 4), 14 (84) (art. 55): 453-455. 
Günther, A. (1880). Report on the shore fishes procured during the voyage of H. M. S. Challenger in the years 1873-1876, In: Report on the scientific results of the voyage of H. M. S. Challenger during the years 1873-76. Zoology. Volume 1 (pt 6): 1-82.

Haly, A. (1875). Descriptions of new species of fish in the collection of the British Museum. Annals and Magazine of Natural History (Series 4), 15 (88) (art. 35): 268-270.

Hamilton, F. (1822). An account of the fishes found in the river Ganges and its branches. Printed for A. Constable and company, Edinburgh and Hurst, Robinson, and Co., London. vii + 405 pp.

Hardenberg, J. D. F. (1936). On a collection of fishes from the estuary and the lower and middle course of the River Kapuas (W. Borneo). Treubia, Buitenzorg, 15 (pt 3): 225254.

Hardenberg, J. D. F. (1941). Fishes of New Guinea. Treubia, Buitenzorg, 18 (pt 2): 217-231.

Hase, A. (1914). Die Fische der Deutschen Grenzexpedition 1910 in das Kaiser-WilhelmsLand, Neu Guinea. Jenaische Zeitschrift für Naturwissenschaft, 51: 525-548.

Hasselquist, F. (1762). D. Friedrich Hasselquists, der Akademien der Wissenschaften zu Stockholm und Upsala Mitglieds, Reise nach Palästina in dem Jahren von 1749 bis 1752. Auf Befehl Ihro Majestät der Königinn von Schweden herausgegeben von Carl Linnaeus. Aus dem Schwedischen [übersetzt von T. H. Gadebusch]. Johann Christian Koppem, Rostock, i-xviii + 1-606. [Placed on Official Index of Rejected and Invalid Works in Zoology by ICZN Dir. 32 (1956).]

Hatai, K. (1965). Some fish otoliths from northeast Honshu. Scientific Reports of the Tokoho University, 37: 63-78.

Heckel, J. J. (1840). Johann Natterer's neue Flussfische Brasilien's nach den Beobachtungen und Mittheilungen des Entdeckers beschrieben (Erste Abteilung, Die Labroiden). Annalen des Wiener Museums der Naturgeschichte, 2: 325-471.

Heemstra, P. C. and Heemstra, E. (2004). Coastal fishes of southern Africa. NISC and SAIAB, Cape Town. xxiv + 488 pp.

Heller, E. and Snodgrass, R. E. (1903). Papers from the Hopkins Stanford Galapagos expedition, 1898-1899. XV. New fishes. Proceedings of the Washington Academy of Sciences, 5: 189-229.

Herre, A. W. C. T. (1932). Fishes from Kwangtung Province and Hainan Island, China. Lingnan Science Journal, Canton, 11 (3): 423-443.

Herre, A. W. C. T. (1935). A new sciaenid from southeastern China. Lingnan Science Journal, Canton, 14 (4): 603-604.

Hildebrand, S. F. (1946). A descriptive catalog of the shore fishes of Peru. Bulletin of the United States National Museum, 189: 1-530.

Hoese, D. F. (2006). Eleotridae. Gudgeons, sleepers, In: Beasley, O. L. and Wells, A. (Eds.), Zoological Catalogue of Australia. Volume 35, Part 3 (3). ABRS and CSIRO Publishing, Australia. pp. 1596-1611.

Holbrook, J. E. (1848). Southern ichthyology, or, a description of the fishes inhabiting the waters of South Carolina Georgia and Florida. No. III. Wiley and Putnam, New York and London. $182 \mathrm{pp}$.

Holbrook, J. E. (1855). Ichthyology of South Carolina. John Russell, Charleston S. C. 184 pp.

Holbrook, J. E. (1860). Ichthyology of South Carolina. Second Edition. John Russell, Charleston S. C. 205 pp. 
Hora, S. L. (1924). Zoological results of a tour in the Far East. Fish of the Tale Sap, Peninsular Siam. Parts I, II. Memoirs of the Asiatic Society of Bengal, 6 (pt 9): 461-501.

Houttuyn, M. (1782). Beschryving van eenige Japanese Visschen, en andere zee-schepzelen. Verhandelingen der Hollandsche Maatschappij der Wetenschappen, Haarlem, 20 (pt 2): 311-350.

Hubbs, C. L. (1921). Description of a new sciaenoid fish from Santa Catalina Island, California. Occasional Papers of the Museum of Zoology University of Michigan, 92: 13.

Humphries P. and Walker, K. (2013). Ecology of freshwater Australian Fishes. CSIRO Publishing, Collingwood, Australia. 440 pp.

Hutchings, K. and Griffiths, M. H. (2005). Identity and distribution of southern African sciaenid fish species of the genus Umbrina. African Journal of Marine Science, 27 (1): 121. https://doi.org/10.2989/18142320509504064

Hutchins, J. B. (2001a). Biodiversity of shallow reef fish assemblages in Western Australia using a rapid censuring technique. Records of the Western Australian Museum, 20 (pt 3): 247-270.

Hutchins, J. B. (2001b). Checklist of the fishes of Western Australia. Records of the Western Australian Museum, Suppl. 63: 9-50.

Iwatsuki, Y., Jawad, L. A. and Al-Mamry, J. M. (2012). Johnius (Johnius) majan sp. nov., a sciaenid fish (Pisces: Sciaenidae) from Oman, Indian Ocean. Ichthyological Research, 59: 151-155. https://doi.org/10.1007/s10228-011-0265-8

Jacquinot, H. and Guichenot, A. (1853). Reptiles et poisons, In: Dumont d'Urville, J., Voyage au Pôle Sud et dans l'Oceanie sur les corvettes L'Astrolabe et La Zélée, executé par ordre du Roi pendant les années 1837-1838-1839-1840, sous le commandement de M.J. Dumont D'Urville, Capitaine de vaisseau. Zoologie. Tome troisième (pt 2), Gide Editeur, Paris. pp. 1-56.

Jenyns, L. (1840-42). The zoology of the voyage of H. M. S. Beagle, under the command of Captain Fitzroy, R. N., during the years 1832 to 1836. Part IV. Fish, by the Rev. Leonard Jenyns, M. A., F. L. S., etc. Smith, Elder, and Co., London, xvi + 172 pp. [Sherborn 1897 dates to Jan. 1840 (pp. 1-32): June 1840 (33-64), Apr. 1841 (65-96), Apr. 1842 (97172).]

Jiménez-Prado, P. J. and Béarez, P. (2004). Peces Marinos del Ecuador Continental / Marine fishes of continental Ecuador. Tomo II. SIMBIOE/NAZCA/IFEA, Quito, Ecuador. 401 pp.

Johnson, J. W. (1999). Annotated checklist of the fishes of Moreton Bay, Queensland, Australia. Memoirs of the Queensland Museum, 43 (2): 709-762.

Johnson, J. W. and Randall, J. E. (2008). Synclidopus hogani, a new species of soleid fish from northeastern Queensland, Australia. Memoirs of the Queensland Museum 52 (2): 245-254.

Jordan, D. S. (1876). Manual of the vertebrates of the northern United States, including the district east of the Mississippi River and north of North Carolina and Tennessee, exclusive of marine species. Chicago, Jansen, McClurg and Company. 407 pp.

Jordan, D. S. (1890). Catalogue of fishes collected at Port Castries, St. Lucia, by the steamer Albatross, November, 1888. In: Scientific Results of explorations by the U. S. Fish Commission steamer Albatross. No. IX. Proceedings of the United States National Museum, 12 (789): 645-652. 
Jordan, D. S. (1896). Notes on fishes, little known or new to science. Proceedings of the California Academy of Sciences (Series 2), 6: 201-244.

Jordan, D. S. (1917). The genera of fishes, from Linnaeus to Cuvier, 1758-1833, seventy-five years, with the accepted type of each. A contribution to the stability of scientific nomenclature. (Assisted by Barton Warren Evermann.). Leland Stanford Jr. University Publications, University Series, 27: 1-161.

Jordan, D. S. (1919). The genera of fishes, part III, from Guenther to Gill, 1859-1880, twentytwo years, with the accepted type of each. A contribution to the stability of scientific nomenclature. Leland Stanford Junior University Publications, University Series, 39: 285-410.

Jordan, D. S. and Bollman, C. H. (1890). Descriptions of new species of fishes collected at the Galapagos Islands and along the coast of the United States of Colombia, 1887-'88. In: Scientific results of explorations by the U. S. Fish Commission steamer Albatross. Proceedings of the United States National Museum, 12 (770): 149-183.

Jordan, D. S. and Dickerson, M. C. (1908). Notes on a collection of fishes from the Gulf of Mexico at Vera Cruz and Tampico. Proceedings of the United States National Museum, 34 (1592): 11-22.

Jordan, D. S. and Eigenmann, C. H. (1889). A review of the Sciaenidae of America and Europe. United States Commission of Fish and Fisheries. Report of the Commissioner 14: 343-451.

Jordan, D. S. and Evermann, B. W. (1898). The fishes of North and Middle America, a descriptive catalogue of the species of fish-like vertebrates found in the waters of North America, north of the Isthmus of Panama. Part II. Bulletin of the United States National Museum, 47: i-xxx + 1241-2183.

Jordan, D. S. and Evermann, B. W. (1900). The fishes of North and Middle America, a descriptive catalogue of the species of fish-like vertebrates found in the waters of North America, north of the Isthmus of Panama. Part IV. Bulletin of the United States National Museum, 47: i-ci + 3137-3313.

Jordan, D. S. and Evermann, B. W. (1927). New genera and species of North American Fishes. Proceedings of the California Academy of Sciences (Series 4), 16 (15): 501-507.

Jordan, D. S. and Evermann, B. W. (1896). A check-list of the fishes and fish-like vertebrates of North and Middle America. United States Commission of Fish and Fisheries, Report of the Commissioner, 21 (Appendix 5): 207-584.

Jordan, D. S. and Gilbert, C. H. (1880). Notes on a collection of fishes from San Diego, California. Proceedings of the United States National Museum, 3 (106): 23-34.

Jordan, D. S. and Gilbert, C. H. (1882a). Descriptions of nineteen new species of fishes from the Bay of Panama. Bulletin of the U. S. Fish Commission, 1: 306-335.

Jordan, D. S. and Gilbert, C. H. (1882b). Notes on a collection of fishes made by Lieut. Henry E. Nichols, U. S. N., on the west coast of Mexico, with descriptions of new species. Proceedings of the United States National Museum, 4 (221): 225-233.

Jordan, D. S. and Gilbert, C. H. (1882c). List of fishes collected by Lieut. Henry E. Nichols, U. S. N., in the Gulf of California and on the west coast of Lower California, with descriptions of four new species. Proceedings of the United States National Museum, 4 (227): 273-279.

Jordan, D. S. and Gilbert, C. H. (1882d). Descriptions of thirty-three new species of fishes from Mazatlán, Mexico. Proceedings of the United States National Museum, 4 (237): $338-365$. 
Jordan, D. S. and Gilbert, C. H. (1882e). Description of five new species of fishes from Mazatlán, Mexico. Proceedings of the United States National Museum, 4 (254): 458463.

Jordan, D. S. and Gilbert, C. H. (1883). Synopsis of the fishes of North America. Bulletin of the United States National Museum, 16: i-liv + 1-1018.

Jordan, D. S. and Gilbert, C. H. (1884-85). Description of Sciaena sciera, a new species of Sciaena from Mazatlan and Panama. Proceedings of the United States National Museum, 7 (452): 480-482. [p. 480 with new taxon name and part of diagnosis published on 23 Oct. 1884, pp. 481-482 on 25 Feb. 1885.]

Jordan, D. S. and Gunn, J. A. (1898). List of fishes collected at the Canary Islands by Mr. O. F. Cook, with descriptions of four new species. Proceedings of the Academy of Natural Sciences of Philadelphia, 50: 339-347.

Jordan, D. S. and Metz, C. W. (1913). A catalog of the fishes known from the waters of Korea. Memoirs of the Carnegie Museum, 6 (1): 1-65.

Jordan, D. S. and Richardson, R. E. (1909). A catalog of the fishes of the island of Formosa, or Taiwan, based on the collections of Dr. Hans Sauter. Memoirs of the Carnegie Museum, 4 (4): 159-204.

Jordan, D. S. and Seale, A. (1905a). List of fishes collected in 1882-83 by Pierre Louis Jouy at Shanghai and Hongkong, China. Proceedings of the United States National Museum, 29 (1433): 517-529.

Jordan, D. S. and Seale, A. (1905b). List of fishes collected at Hong Kong by Captain William Finch, with description of five new species. Proceedings of the Davenport Academy of Sciences (Iowa), 10: 1-17.

Jordan, D. S. and Snyder, J. O. (1900). A list of fishes collected in Japan by Keinosuke Otaki, and by the United States steamer Albatross, with descriptions of fourteen new species. Proceedings of the United States National Museum, 23 (1213): 335-380.

Jordan, D. S. and Starks, E. C. (1904). The fishes of Panama Bay. Memoirs of the California Academy of Sciences, 4: 1-304.

Jordan, D. S. and Starks, E. C. (1905). On a collection of fishes made in Korea, by Pierre Louis Jouy, with descriptions of new species. Proceedings of the United States National Museum, 28 (1391): 193-212.

Jordan, D. S. and Starks, E. C. (1906). Notes on a collection of fishes from Port Arthur, Manchuria, obtained by James Francis Abbott. Proceedings of the United States National Museum, 31 (1493): 515-526.

Jordan, D. S. and Thompson, W. F. (1911). A review of the sciaenoid fishes of Japan. Proceedings of the United States National Museum, 39 (1787): 241-261.

Kabir, N., Shamsunnahar, Abdul Baki, M., Ahmed, S. and Ahmed, Md. S. (2018). New record of the squaretail kob, Argyrosomus thorpei Smith, 1977 (Perciformes: Sciaenidae) from the coast of Bay of Bengal, Bangladesh. International Journal of Fisheries and Aquatic Studies, 6 (5): 195-197.

Kendall, W. C. and Radcliffe, L. (1912). The shore fishes, In: Reports on the scientific results of the expedition to the eastern tropical Pacific, in charge to Alexander Agassiz, by the U. S. Fish Commission steamer "Albatross," from October, 1904 to March, 1905, Lieut. Commander L. M. Garrett, U. S. N. Commanding. Memoirs of the Museum of Comparative Zoology, 35 (3): 75-171. 
Khan, R. A. (1993). Fish faunal resources of Sunderban estuarine system with special reference to the biology of some commercially important species. Records of the Zoological Survey of India, Occasional Paper, 209: 1-150.

Klunzinger, C. B. (1879). Die v. Müller'sche Sammlung australischer Fische. Anzeiger der Kaiserlichen Akademie der Wissenschaften, Wien, Mathematisch-Naturwissenschaftliche Classe, 16 (22): 254-261.

Kong, I. and Valdés, J. 1990. Sciaénidos de Chile: análisis taxonómico y morfológico. EstudiosOceanológicos, 9: 13-56.

Kottelat, M. (2013). The fishes of the inland waters of Southeast Asia, a catalogue and core bibliography of the fishes known to occur in freshwaters, mangroves and estuaries. The Raffles Bulletin of Zoology, 2013 (Suppl. 27): 1-663.

Krishnan, S. and Mishra, S. S. (1993). On a collection of fish from Kakinada - Gopalpur sector of the east coast of India. Records of the Zoological Survey of India, 93 (1, 2): 201-240.

Lacepède, B. G. E. (1801). Histoire naturelle des poissons. Tome troisième. Plassan, Paris, lxvi +558 pp.

Lacepède, B. G. E. (1802). Histoire naturelle des poissons. Tome deuxième. Plassan, Paris. lxiv +632 pp.

Lacepède, B. G. E. (1802). Histoire naturelle des poissons. Tome quatrième. Plassan, Paris. lxiv $+728 \mathrm{pp}$.

Lacepède, B. G. E. (1803). Histoire naturelle des poissons. Tome cinquième. Plassan, Paris. lxviii +803 pp. + index.

Lal Mohan, R. S. (1983). Sciaenidae, In: Fischer, W. and Bianchi, G. (Eds.), F. A. O. Species Identification Sheets for Fishery purposes, Western Indian Ocean. FAO, Rome. $9+62$ unnumbered pp.

Lal Mohan, R. S. (1984). Systematic position of the sciaenid Corvina carouna Cuvier, 1830. Indian Journal Fishery, 31 (3): 374-377.

Lal Mohan, R. S. (1969). On three new genera of sciaenid fishes (Pisces, Sciaenidae) from India. Current Science, 38 (12): 295-296.

Lal Mohan, R. S. (1971). A new species of sciaenid fish Johnius mannarensis from the southeast coast of India. Journal of the Marine Biological Association of India, 11 (1-2): 320323.

Lal Mohan, R. S. (1972). A synopsis of the Indian genera of the fishes of the family Sciaenidae. Indian Journal of Fisheries, 16 (1-2): 82-98.

Lal Mohan, R. S. (1976). Two new species of sciaenid fishes Johnius elongatus and Johnieops macrorhynus from India. Matsya, 1: 19-25.

Larrañaga, D. A. (1923). Escritos de Don Dámaso Antonio Larrañaga. Volume 2. Instituto Histórico y Geográfico del Uruguay (Imprenta Nacional), Montevideo. 512 pp.

Larson, H. K, Jaafar, Z. and Lim, K. K. P. (2008). An annotated checklist of the gobioid fishes of Singapore. The Raffles Bulletin of Zoology, 56 (1): 135-155.

Larson, H. K. and Murdy, E. O. (2001). Families Eleotridae, Gobiidae, In: Carpenter, K. E. and Niem, V. H. (Eds.), Species identification guide for fishery purposes. The living marine resources of the western central Pacific. Bony fishes part 4 (Labridae to Latimeriidae), estuarine crocodiles, sea turtles, sea snakes and marine mammals. FAO, Rome. pp. 3574-3603. 
Larson, H. K. and Pidgeon, B. (2004). New records of freshwater fishes from East Timor. The Beagle, Records of the Museums and Art Galleries of the Northern Territory, 20: 195198.

Larson, H. K. and Sparks, J. S. (2017). "Giuris margaritacea". The IUCN Red List of Threatened Species. 2017e.T196317A123380986.

Lesueur, C. A. (1822). Description of three new species of the genus Sciaena. Journal of the Academy of Natural Sciences of Philadelphia, 2: 251-256.

Lin, S.-Y. (1935). Notes on some important sciaenid fishes of China. Bulletin of the Chekiang Provincial Fisheries Experiment Station, Tinghai, 1 (1): 1-30.

Lin, S.-Y. (1938). Further notes on sciaenid fishes of China. Lingnan Science Journal, Canton, 17 (3): 367-381.

Lin, S.-Y. (1940). Croakers of the South China Sea. Journal of the Hong Kong Fisheries Research Station, 1 (2): 243-254.

Lin, Y.-J., Qurban, M. A., Shen, K. N. and Chao, N. L. (2019). Delimitation of tiger-tooth croaker Otolithes species (Teleostei: Sciaenidae) from the western Persian Gulf using an integrative approach, with a description of Otolithes arabicus sp. nov. ZoologicalStudies, 58: 1-18. https://doi.org/10.6620/ZS.2019.58-10

Linnaeus, C. (1754). Museum S:ae R:ae M:tis Adolphi Friderici Regis Svecorum, Gothorum, Vandalorumque Haer. Norv. Duc. Slesv. Hols. Storm. Ditm. Com. Oldenb. Delmenhorstiae andc. andc. in quo Animalia rariora imprimis et exotica, Quadrupedia, Aves, Amphibia, Piscis, Insecta, Vermes describuntur et determinantur, latine et svetice cum iconibus. Tomus I. Holmiae, Typographia Regia. XXX + 103 pp.

Linnaeus, C. (1758). Systema naturae per regna tria naturae, secundum classes, ordines, genera, species, cum characteribus, differentiis, synonymis, locis. Tomus I. Editio decima, reformata. Laurentii Salvii, Holmiae. 823 pp.

Linnaeus, C. (1764). Museum S:ae R:ae M:tis Adolphi Friderici Regis Svecorum, Gothorum, Vandalorumque andc. andc. andc. in quo Animalia rariora imprimis and exotica, Aves, Amphibia, Piscis describuntur. Tomi secundi Prodromus. Laurentii Salvii, Holmiae. 110 pp.

Linnaeus, C. (1766). Systema naturae per regna tria naturae, secundum classes, ordines, genera, species, cum characteribus, differentiis, synonymis, locis. Laurentii Salvii, Holmiae. 12th ed., 1 (pt 1): 1-532.

Lo, P.-C., Liu, S.-H., Chao, N. L., Nunoo, F. K. E., Mok, H.-K. and Chen, W.-J. (2015). A multi-gene dataset reveals a tropical New World origin and early Miocene diversification of croakers (Perciformes, Sciaenidae). Molecular Phylogenetics and Evolution, 88: 132143. https://doi.org/10.1016/j.ympev.2015.03.025

Lo, P.-C., Liu, S.-H., Mohd Nor, S. A. and Chen, W.-J.(2017). Molecular exploration of hidden diversity in the Indo-West Pacific sciaenid clade. PLoS ONE, 12 (4): e0176623, 1-21. https://doi.org/10.1371/journal.pone.0176623

López H. L., Menni, R. C. and Miquelarena, A. M. (2003). Lista critica comentada de los peces de agua dulce del Río de la Plata. Ministerio de Vivienda Ordinamiento Territorial y Medio Ambiente, Montevideo, Uruguay. 74 pp.

López S., M. I. (1980). Umbrina bussingi, a new sciaenid fish from the tropical eastern Pacific Ocean. Revista de Biología Tropical, 28 (1): 203-208.

López S., M. I. and Bussing, W. A. (1982). Lista provisional de los peces marinos de la costa Pacifica de Costa Rica. Revista de Biología Tropical, 30 (1): 5-26. 
López, H. L., Morgan, C. C. and Montenegro, M. J. (2002). Ichthyological ecoregions of Argentina. Museo del Plata, Buenos Aires. 68 pp.

MacDonagh, E. J. (1931). Sobre la corvina de San Blas: Macropogon patagonensis. Physis (Buenos Aires), 10: 409-410.

MacDonagh, E. J. (1934). Nuevos conceptos sobre la distribución geográfica de los peces Argentinos basados en expediciones del Museo de la Plata. Revista del Museo de La Plata, 34: 21-170.

Macleay, W. (1880). On two hitherto undescribed fishes well-known in the Sydney market. Proceedings of the Linnean Society of New South Wales, 5 (pt 1): 48-49.

Macleay, W. (1883). Notes on a collection of fishes from the Burdekin and Mary rivers, Queensland. Proceedings of the Linnean Society of New South Wales, 8 (pt 2): 199-213.

Manilo, L. G. and Bogorodsky, S. V. (2003). Taxonomic composition, diversity and distribution of coastal fishes of the Arabian Sea. Journal of Ichthyology, 43 (suppl. 1): S75-S149.

Marceniuk, A. P., Molina, E. G., Caires, R. A., Rotundo, M. M., Wosiacki, W. B. and Oliveira, C. (2019). Revision of Bairdiella (Sciaenidae, Perciformes) from the western South Atlantic, with insights into its diversity and biogeography. Neotropical Ichthyology, 17 (1): 1-18. http://dx.doi.org/10.1590/1982-0224-20180024

Marggraf von Liebstadt, G. (1648). Historiæ rerum naturalium brasiliæ libri octo. In: W. Piso and G. Marggraf, Historiae naturalis brasiliae, auspicio et beneficio illustriss. I. Mavritii Com. Nassau ... in qua non tantum plantce et animalia, sed in indigenarum morbi, ingenia et mores describuntur et iconibus supra quingentas illustrantur. F. Hackius, Lugduni Batavorum et L. Elzevir, Amstelodami. xii (unnumbered) + 293 pp.

Matsubara, K. (1937). Sciaenoid fishes found in Japan and its adjacent waters. Journal of the Imperial Fisheries Institute, 32 (2): 27-92.

Matsunuma, M., Motomura, H., Matsuura, K., Shazili, N. A. M. and Ambak, M. A. (Eds.) (2011). Fishes of Terengganu - east coast of Malay Peninsula, Malaysia. National Museum of Nature and Science, University Malaysia Terengganu and Kagoshima University Museum, Kagoshima. ix + 251 pp.

McDowall, R. M. (1997). The evolution of diadromy in fishes (revisited) and its place in phylogenetic analysis. Reviews in Fish Biology and Fisheries, 7(4): 443-462. https://doi.org/10.1023/A: 1018404331601

McFrland, W. N. (1963). Seasonal changes in the number and the biomass of fishes from the surf at Mustand Island, Texas. Publications of the Institute of Marine Science, University of Texas, 9: 91-105.

McMahan, C. D., Matamoros, W. A., Álvarez Calderón, F. S., Henríquez, W. Y., Recinos, H. M., Chakrabarty, P., Barraza, E. and Herrera, N. (2013). Checklist of the inland fishes of El Salvador. Zootaxa, 3608 (6): 440-456. http://dx.doi.org/10.11646/zootaxa.3608.6.2

McPhail, J. D. (1958). Key to the croakers (Sciaenidae) of the eastern Pacific. Institute of Fisheries, University of British Columbia Museum Contributions, 2: 1-20.

McPhail, J. D. (1961). A review of the tropical eastern Pacific species of Pereques (Sciaenidae). Copeia, 1961 (1): 27-32. http://dx.doi.org/10.2307/1440167

Meek, S. E. and Hildebrand, S. F. (1925). The marine fishes of Panama. Part II. Field Museum of Natural History, Publications, Zoölogical Series, 15: i-xix + 331-707.

Meléndez, C. R., Galvez, H. O. and Cornejo, C. A. (1993). Catálogo colección de peces depositada en el Museo Nacional de Historia Natural de Chile. Publicacion Ocasional Museo Nacional de Historia Natural, Santiago, Chile, 47: i-xviii + 1-224. 
Metzelaar, J. (1919). Report on the fishes, collected by Dr. J. Boeke in the Dutch West Indies 1904-1905, with comparative notes on marine fishes of tropical West Africa. F.J. Belanfante, 's-Gravenhage, Netherlands. 314 pp.

Miesen, F. W., Droppelmann, F., Hüllen, S., Hadiaty, R. K. and Herder, F. (2016). An annotated checklist of the inland fishes of Sulawesi. Bonn zoological Bulletin, 64 (2): 77-106.

Miller, D. J. and Lea, R. N. (1972). Guide to the coastal marine fishes of California. Fish Bulletin of the Department of Fish and Game California, 157: 1-235.

Miller, G. C. and Woods, L. P. (1988). A new species of sciaenid fish, Pareques iwamotoi, from the western Atlantic, with color description of prejuvenile and juvenile Pareques acuminatus and Pareques umbrosus. Bulletin of Marine Science, 43 (1): 88-92.

Miller, R. V. (1971). A new sciaenid fish (Pisces, Umbrinini) with a single mental barbel, from the southern Caribbean. Copeia, 1971 (2): 300-306. http://dx.doi.org/10.2307/1442831

Miranda Ribeiro, A. de (1913). Sciaenidae, In: Fauna brasiliense. Peixes. V. [Eleutherobranchios aspirophoros]. Physoclisti. Arquivos do Museu Nacional de Rio de Janeiro, 17, 46 pp. [Issued in parts by families. Not continuously paginated].

Mitchill, S. L. (1814). Report, in part, of Samuel L. Mitchill, M. D., Professor of Natural History, andc, on the fishes of New-York. D. Carlisle, New York. 28 pp.

Mitchill, S. L. (1815). The fishes of New York described and arranged. Transactions of the Literary and Philosophical Society of New York, 1 (art. 5): 355-492.

Moreau, É. (1874). Poissons de France. Notes sur quelques espèces nouvelles des côtes de l'océan. Revue et Magasin de Zoologie (Sér. 3), 2: 115-119, Pls. 14-15.

Moreno, C. (1970). Micropogon manni n. sp. (Sciaenidae, Teleostomi). Noticiaro mensual, Museo Nacional de Historia Natural (Santiago de Chile), 15 (170): 5-10.

Müller, J. and Troschel, F. H. (1849). Fische, In: Schomburgk, R. (Ed.), Reisen in BritischGuiana in den Jahren 1840-44. Im Auftrag Sr. Mäjestat des Königs von Preussen ausgeführt von Richard Schomburgk. Band 3. Versuch einer Fauna und Flora von Britisch-Guiana. Verlagsbuchhundlung von J. J. Weber, Leipzig. pp. 618-644.

Munro, I. S. R. (1964). Additions to the fish fauna of New Guinea. Papua New Guinea Agricultural Journal, 16 (4): 141-186.

Mutia, M. T. M., Muyot, M. C., Torrs Jr, F. B. and Faminialagao, C. M. (2018). Status of Taal Lake Fishery Resources with Emphasis on the Endemic Freshwater Sardine, Sardinellatawilis (Herre, 1927). The Philippine Journal of Fisheries, 25 (1): 128-135. http://dx.doi.org/10.31398/tpjf/25.1.2017C0017

Myers, R. F. (1999). Micronesian reef fishes. A comprehensive guide to the coral reef fishes of Micronesia. Third Revised Edition. Coral Graphics, Guam. vi + 330 pp.

Myers, R. F. and Donaldson, T. J. (2003). The fishes of the Mariana Islands. Micronesica, 35/36: 598-652.

Nakashima, S. (1941). Algunos peces del Orient peruano. Boletín del Museo de Historia Natural "Javier Prado", 5 (16): 61-78.

Nelson, J. S., Crossman, E. J., Espinosa Pérez, H., Findley, L. T., Gilbert, C. R., Lea, R. N. and Williams, J. D. (2004). Common and scientific names of fishes from the United States, Canada, and Mexico. Sixth Edition. Special Publication No. 29. American Fisheries Society, Bethesda, Maryland. ix + 386 pp.

Nelson, J. S., Grande, T. C. and Wilson, M. V. H. (2016). Fishes of the World. Fifth Edition. John Wiley and Sons, Hoboken, New Jersey. v-xli + 707 pp. 
Ng, H. H., Tan, H. H. and Lim, K. K. P. (1999). The inland fishes of Pulau Tioman, peninsular Malaysia. The Raffles Bulletin of Zoology, 6: 169-187.

Nichols, J. T. (1913). Notes on Teleosts collected by Mr. Roy C. Andrews in Japan, with descriptions of two new species. Bulletin of the American Museum of Natural History, 32: $179-183$.

Norman, J. R. (1922). Three new fishes from Zululand and Natal, collected by Mr. H. W. Bell Marley; with additions to the fish fauna of Natal. Annals and Magazine of Natural History (Series 9), 9 (52): 318-322.

Norman, J. R. (1935). Coast fishes. Part I. The South Atlantic. Discovery Reports, 12: 1-58.

Ogilby, J. D. (1908). Descriptions of new Queensland fishes. Proceedings of the Royal Society of Queensland, 21: 87-98.

Ogilby, J. D. (1918). Edible fishes of Queensland. Parts X through XIV. Memoirs of the Queensland Museum, 6: 45-90.

Oken, L. (1817). V. Kl. Fische. Isis (Oken), 1 (pt 8) (148): 1779-1782 [slip for 1179-1182; see Gill 1903 for correct pagination].

Opinion 988. (1972). Sciaena Linnaeus, 1758 (Pisces), designation of a type-species under the plenary power. Bulletin of Zoological Nomenclature, 29 (3): 123-124.

Osório, B. (1909). Peixes d'agua doce da Guiné Portugueza. Memorias do Museu Bocage, Lisboa, 1: 95-107.

Ostroumoff, A. A. (1896). Zwei neue Relicten-Gattungen im Azow'schen Meere. Zoologischer Anzeiger, 19 (493): 30.

Oyarzun, C., Herrera, G. and Lavenberg, R. J. (1985). Status taxónomicos de la corvina Cilus montti Delfin (1900 (Pisces, Sciaenidae). Biologia Pesquera, 14: 40-45.

Page, L. M., Espinosa-Pérez, H., Findley, L. D., Gilbert, C. R., Lea, R. N., Mandrak, N. E., Mayden, R. L. and Nelson, J. S. (2013). Common and scientific names of fishes from the United States, Canada, and Mexico. Seventh Edition. Special Publication No. 34. American Fisheries Society, Bethesda, Maryland. xii +384 pp.

Pallas, P. S. (1814). Zoographia Rosso-Asiatica, sistens omnium animalium in extenso Imperio Rossico et adjacentibus maribus observatorum recensionem, domicilia, mores et descriptiones anatomen atque icones plurimorum. Volumen tertium. Officina Caes. Academiae Scientiarum Impress, Petropoli. vii + 428 pp. + CXXV pp (index). [Date of publication fixed in Opinion 212, ICZN]

Pappe, C. W. L. (1853). Synopsis of the Edible Fishes at the Cape of Good Hope. Van de Sandt de Villiers and Tier, Cape Town. 34 pp.

Parenti, P. and Randall, J. E. (2000). An annotated checklist of the species of the Labroid fish families Labridae and Scaridae. Ichthyological Bulletin of the J. L. B. Smith Institute of Ichthyology, 68: 1-97.

Parenti, P. (2013). Perca luth Walbaum, 1792, a senior synonym of the meagre, Argyrosomus regius (Asso, 1801). (Perciformes, Sciaenidae), invalidated by "reversal of precedence". $\begin{array}{lllll}\text { Journal of } & \text { FisheriesSciences.com, } & 7 & \text { (1): 79-82. }\end{array}$ http://dx.doi.org/10.3153/jfscom.2013008

Parenti, P. (2019a). An annotated checklist of the fishes of the family Haemulidae (Teleostei, Perciformes). Iranian Journal of Ichthyology, 6 (3): 150-196. http://dx.doi.org/10.22034/iji.v6i2.408

Parenti, P. (2019b). The status of the fishes described from Sicily by Rafinesque. FishTaxa, 4 (1): 99-124. 
Pequeño, G. (1989). Peces de Chile. Lista sistemática revisada y comentada. Revista de Biologia Marina, Valparaiso, 24 (2): 1-132.

Peters, W. (1855). Übersicht der in Mossambique beobachteten Seefische. Bericht über die zur Bekanntmachung geeigneten Verhandlungen der Königlichen Preussischen Akademie der Wissenschaften zu Berlin, 1855: 428-466.

Peters, W. (1881). Über die von der chinesischen Regierung zu der internationalen FischereiAustellung gesandte Fischsammlung aus Ningpo. Monatsberichte der Königlichen Preussischen Akademie der Wissenschaften zu Berlin, 45: 921-927.

Poey, F. (1860). XLIX. Poissons de Cuba, In: Poey, F. (Ed.), Memorias sobra la historia natural de la Isla de Cuba, acompañadas de sumarios Latinos y extractos en Francés. Tomo 2. Viuda de la Barcina, La Habana. pp. 115-356.

Poey, F. (1875). Poissons de l'île de Cuba. Espèces nouvelles décrites. Annals of the Lyceum of Natural History of New York, 11 (3-4) (art. 6): 58-70.

Poey, F. (1881). IV. Peces, In: Gundlach, J. (Ed.), Apuntes para la fauna puerto-riqueña. Tercera parte. Anales de la Sociedad Española de Historia Natural, Madrid. 10, pp. 317350.

Poll, M. (1954). Poissons IV. Téléostéens Acanthoptérygiens (première partie). Résultats Scientifiques. Expédition Océanographique Belge dans les Eaux Côtières Africaines de l'Atlantique Sud (1948-1949). Institut Royale Science Naturelle du Belgique, 4 (3A): 1390.

Pusey, B. J., Burrows, D. W., Kennard, M. J., Perna, C. N., Unmack, P. J., Allsop, Q. and Hammer, M. P. (2017). Freshwater fishes of Northern Australia. Zootaxa, 4253 (1): 1104. http://dx.doi.org/10.11646/zootaxa.4253.1.1

Quoy, J. R. C. and Gaimard, J. P. (1824). Description des Poissons. Chapter IX, In: de Freycinet, L. (Ed.), Voyage autour du Monde, entrepris par ordre du Roi, sous le ministère et conformément aux instructions de S. Exc. M. le Vicomte du Bouchage, Sécretaire d'État au Département de la Marine, exécuté sur les corvettes de L. M. "L'Uranie" et "La Physicienne," pendant les années 1817, 1818, 1819 et 1820; publié sous les auspices de S. E. M. le ComteCorbière, Sécretaire d'Étatde l'Interieur, pour la partie historique et les sciences naturelles et de S. E. M. le Marquis de ClermontTonnerre, Sécretaire d'État de la Marine et des Colonies, pour la partie nautique. Pillet Aîné, Paris. pp. 192-401.

Rafinesque C. S. (1810a). Caratteri di alcuni nuovi generi e nuove specie di animali e piante della Sicilia con varie osservazioni sopra i medesimi. Sanfilippo, Palermo. 105 pp.

Rafinesque C. S. (1810b). Indice d'ittiologia siciliana; ossia catalogo metodico dei nomi latini italiani e siciliani dei pesci che si rinvengono in Sicilia disposti secondo un metodo naturale e seguito da un appendice che contiene la descrizione de alcuni nuovi pesci siciliani. Giovanni del Nobolo, Messina. 70 pp.

Rafinesque C. S. (1815). Analyse de la nature, ou tableau de l'univers et des corps organisés. Imprimerie Jean Barravecchia, Palermo, Italia. 224 pp.

Rafinesque C. S. (1819). Prodrome de 70 nouveaux genres d'animaux découverts dans l'intérieur des États-Unis d'Amérique, durant l'année 1818. Journal de Physique, de Chimie et d'Histoire Naturelle, 88: 417-429.

Ramírez-Hernández, E. and Gonzáles-Pagés, A. (1976). Catálogo de peces marinos mexicanos. Instituto Nacional de Pesca, México. 462 pp. 
Ramsay, E. P. and Ogilby, J. D. (1887). On an undescribed Sciaena from the New South Wales coast. Proceedings of the Linnean Society of New South Wales (Series 2), 1 (pt 3): 941-942.

Randall, J. E. (1995). Coastalfishes of Oman. Crawford House Publishing Pty Ltd, Bathurst, Australia. xvi +439 pp.

Randall, J. E. (2005). Reef and shore fishes of the South Pacific. New Caledonia to Tahiti and the Pitcairn Islands. University of Hawai'i Press, Honolulu. xii +707 pp.

Randall, J. E. (2007). Reef and shore fishes of the Hawaiian Islands. University of Hawai'i Press, Honolulu. xiv + 546 pp.

Randall, J. E., Allen, G. R. and Steene, R. C. (1990). Fishes of the Great Barrier Reef and Coral Sea. Crawford House Press, Bathurst NSW. xx + 507 pp.

Randall, J. E., Langston, R. C. and Severns, M. (2006). First record of the labrid fish Cymolutes praetextatus from the Hawaiian Islands. Pacific Science, 60 (4): 549-553. https://doi.org/10.1353/psc.2006.0034

Randall, J. E.and Cervigón M. F. (1968). Un nuevo pez Sciaenidaee del genero Cynoscion de Venezuela y notas sobre $C$. jamaicensis y $C$. obliquatus. Memoria de la Sociedad de Ciencias Naturales La Salle, 27 (78): 176-192.

Randall, J. E. and Lim, K. K. P. (2000). A checklist of the fishes of the South China Sea. The Raffles Bulletin of Zoology, Suppl. 8: 569-667.

Ray, J. (1713). Synopsis methodica piscium. W. Innys, London. $166+$ xiii pp.

Rees, A. J. J., Yearsley, G. K., Gowlett-Holmes, K. and Pogonoski, J. (1999). Codes for Australian Aquatic Biota (on-line version). CSIRO Marine and Atmospheric Research, World Wide Web electronic publication, 1999 onwards. http://www.cmar.csiro.au/caab

Regan, C. T. (1903). Descriptions of new South-American fishes in the collection of the British Museum. Annals and Magazine of Natural History (Series 7), 12 (72) (art. 64): 621-630.

Regan, C. T. (1905). Description de six poissons nouveaux faisant partie de la collection du Musée d'Histoire Naturelle de Genève. Revue Suisse de Zoologie, 13: 389-393.

Reinhardt, J. T. (1851). Nye sydamerikanske Ferskvandsfiske. Videnskabelige Meddelelser fra den Naturhistoriske Forening i Kjфbenhavn, Aaret, 1849 (3-5): 29-57.

Reinhardt, J. T. (1855). Notits om Slaegten Pachyurus Agass. og de dertil hörende Arter. Videnskabelige Meddelelser fra den Naturhistoriske Forening $i$ Kjøbenhavn, Aaret, 1854: 108-112.

Reis, R. E., Kullander, S. O. and Ferraris, Jr., C. J. (Eds.) (2003). Check list of the freshwater fishes of South and Central America. Cloffsca, Edipucrs, Porto Alegre. xi +729 pp.

Richardson, J. (1836). Fauna Boreali-Americana; or the zoology of the northern parts of British America, containing descriptions of the objects of natural history collected on the late northern land expeditions, under the command of Sir John Franklin, R. N. Part Third. Richard Bentley, London. xv +327 pp.

Richardson, J. (1843). Report on the present state of the ichthyology of New Zealand. Reports of the Meetings of the British Association for the Advancement of Science, 12: 12-29.

Richardson, J. (1844). Ichthyology. Part 1, In: Hinds, R. B. (Ed.), The zoology of the voyage of H. M. S. Sulphur, under the command of Captain Sir Edward Belcher, R. N., C. B., F. R. G. S., etc., during the years 1836-42. Smith, Elder and Co, London. pp. 51-70.

Richardson, J. (1846). Report on the ichthyology of the seas of China and Japan. Report of the British Association for the Advancement of Science 15th meeting, [1845]: 187-320. 
Risso A. (1810). Ichthyologie de Nice, ou histoire naturelle des poissons du Département des Alpes Maritimes. F. Schoell, Paris. xxxvi + 388.

Risso A. (1827). Histoire naturelle des principales productions de l'Europe méridionale, et particulièrement de celles des environs de Nice et des Alpes maritimes. F. G. Levrault, Paris and Strasbourg. i-xvi + 1-480.

Rochebrune, A. T. de (1880). Description de quelques nouvelles espèces de poissons propres à la Sénégambie. Bulletin de la Société philomathique de Paris (7th Série), 4: 159-169.

Sánchez-Pinedo, G., Díaz-Viloria, N., Ortiz-Galindo, J. L., Ferreira-Fontoura, N., PerezEnriquez, R., Sánchez-Velasco, L. and De La Cruz-Agüero, J. (2017). Proposed synonymy for Micropogonias altipinnis (Günther 1864), Micropogonias ectenes (Jordan and Gilbert 1882), and Micropogonias megalops (Gilbert 1890). Mitochondrial DNA Part A, 29 (1): 136-146. https://doi.org/10.1080/24701394.2016.1258405

Sasaki, K. (1989). Phylogeny of the family Sciaenidae, with notes on its zoogeography (Teleostei, Perciformes). Memoirs of the Faculty of Fishery Hokkaido University, 36: 1137.

Sasaki, K. (1990). Johnius grypotus (Richardson, 1846), resurrection of a Chinese sciaenid species. Japanese Journal of Ichthyology, 37 (3): 224-229. https://doi.org/10.11369/ jji1950.37.224

Sasaki, K. (1992a). Two new species of Nibea (Sciaenidae) from northern Australia and Papua New Guinea. Japanese Journal of Ichthyology, 39 (1): 1-8. https://doi.org/10.11369/jji1950.39.1

Sasaki, K. (1992b). Two new and two resurrected species of the sciaenid genus Johnius (Johnius) from the west Pacific. Japanese Journal of Ichthyology, 39 (3): 191-199. https://doi.org/10. 11369/jji1950.39.191

Sasaki, K. (1993). Corvina senegalla Cuvier, a senior synonym Pseudotolithus (Pseudotolithus) brachygnathus Bleeker (Sciaenidae, Perciformes). Japanese Journal of Ichthyology, 40 (3): 361-362. https://doi.org/10.11369/jji1950.40.361

Sasaki, K. (1994). Argyrosomus beccus, e new sciaenid from South Africa. Japanese Journal of Ichthyology, 41 (1): 35-38. https://doi.org/10.11369/jji1950.41.35

Sasaki, K. (1995a). A review of the Indo-West Pacific sciaenid genus Panna (Teleostei, Perciformes). Japanese Journal of Ichthyology, 42 (1): 27-38. https://doi.org/10.11369/jji1950.42.27

Sasaki, K. (1995b). Two new species of Atrobucca (Sciaenidae) from the Bay of Bengal. Japanese Journal of Ichthyology, $42 \quad$ (3/4): $269-275$. https://doi.org/10.11369/jji1950.42.269

Sasaki, K. (1996a). Sciaenid fishes of the Indian Ocean (Teleostei, Perciformes). Memoirs of the Faculty of Science, Kochi University (Ser. D) (Biology), 16/17: 83-96.

Sasaki, K. (1996b). Pennahia ovata, a new sciaenid from the Bay of Bengal. Ichthyological Research, 43 (1): 11-16. https://doi.org/10.1007/BF02589605

Sasaki, K. (1997). Resurrection of two East African Species of Johnius (Perciformes, Sciaenidae). Ichthyological Research, 44 (3): 311-315. https://doi.org/10.1007/ BF02678711

Sasaki, K. (1999). Johnius (Johnieops) philippinus, a new sciaenid from the Philippines, with a synopsis of species included in the subgenera Johnieops. Ichthyological Research, 46 (39): 271-280. https://doi.org/10.1007/BF02678513

Sasaki, K. (2001). Family Sciaenidae, In: Carpenter, K. E. and Niem, V. H. (Eds.), Species identification guide for fishery purposes. The living marine resources of the western 
central Pacific. Bony fishes part 3 (Menidae to Pomacentridae). FAO, Rome. pp. 27913380 .

Sasaki, K. and Amaoka, K. (1989). Johnius distinctus (Tanaka, 1916), a senior synonym of J. tingi (Tang, 1937). (Perciformes, Sciaenidae). Japanese Journal of Ichthyology, 35 (4): 466-468. https://doi.org/10.11369/jji1950.35.466

Sasaki, K. and Kailola, P. J. (1988). Three new Indo-Australian species of the sciaenid genus Atrobucca, with a revaluation of generic limit. Japanese Journal of Ichthyology, 35 (3): 261-277. https://doi.org/10.11369/jji1950.35.261

Sasaki, K. and Kailola, P. J. (1991). Johnius (Johnius) laevis, a new sciaenid from northern Australia and Papua New Guinea. Japanese Journal of Ichthyology, 38 (2): 119-123. https://doi.org/10.11369/jji1950.38.119

Sauvage, H. E. (1879). Description de quelques poissons d'espèces nouvelles de la col lection du Muséum d'histoire naturelle. Bulletin de la Société philomathique de Paris (7th Série), 3: 204-209.

Sauvage, H.-E. (1881). Sur une collection de poissons de Swatow. Bulletin de la Société philomathique de Paris (7th Série), 5: 104-107.

Sauvage, H.-E. (1883). Description de quelques poissons de la collection du Muséum d'histoire naturelle. Bulletin de la Société philomathique de Paris (7th Série), 7: 156-161.

Schinz, H. R. (1822). Das Thierreich eingetheilt nach dem Bau der Thiere als Grundlage ihrer Naturgeschichte und der vergleichenden Anatomie. Mit vielen Zusätzenversehen von H. R. Schinz. Zweiter band. Cotta, Stuttgart and Tübingen. xvi + 835 pp.

Schomburgk, R. H. (1843). The fishes of Guiana. Part II, In: Jardine, W. (Ed.), The Naturalists' Library. Ichthyology. Volume 40. W. H. Lizars, Edinburgh. pp. 129-214.

Schultz, L. P. (1945). Three new sciaenid fishes of the genus Ophioscion from the Atlantic coasts of Central and South America. Proceedings of the United States National Museum, 96 (3192): 123-137.

Schultz, L. P. (1949). A further contribution to the ichthyology of Venezuela. Proceedings of the United States National Museum, 99 (3235): 1-211.

Seah, Y. G., Hanafi, N., Mazlan, A. G. and Chao, N. L. (2015). A new species of Larimichthys from Terengganu, east coast of Peninsular Malaysia (Perciformes, Sciaenidae). Zootaxa, 3956 (2): 271-280. http://dx.doi.org/10.11646/zootaxa.3956.2.7

Seale, A. (1910). Fishes of Borneo, with descriptions of four new species. The Philippine Journal of Science, Section D, 5 (4): 263-289.

Shaw, W. (1803). General zoology or systematic natural history by George Shaw, M. D. F. R. $S$. andc. with plates from the first Authorities and most selected specimens engraved principally by Mr. Heath. Volume 4. Part 2. Pisces. G. Kearsley, London. pp. i-xiii + 187-632.

Sherborn, C. D. (1897). Notes on the dates of "The Zoology of the 'Beagle'". Annals and Magazine of Natural History (Series 6), 20 (119): 483.

Sinha, N. K. and Babu Rao, M. (1969). Dendrophysa hooghliensis, a new species of sciaenid fish from India. Copeia, 1969 (1): 77-82. http://dx.doi.org/10.2307/1441699

Sivaperuman, C. and Venkataraman, K. (2018). Indian hotspots, vertebrate faunal diversity, conservation and management. Volume 1. Springer, Singapore. 397 pp.

Sivaperuman, C., Velmurugan, A., Singh, A. K. and Jaisankar, I. (2018). Biodiversity and climate change adaptation in tropical islands. Academic Press, London, San Diego, Cambridge, Oxford. 805 pp. 
Smith, M. M. (1977). A new species of Argyrosomus (Pisces, Sciaenidae) from Natal, South Africa. Journal of Zoology (London), 181 (4): 561-566.

Soares, L. and Casatti, L. (2000). Descrição de duas novas espécies de Sciaenidae (Perciformes) de água doce da bacia Amazônica. Acta Amazonica, 30 (3): 499-513.

Song, Y. S., Kim, J. K., Kang, J. H. and Kim, S. Y. (2017). Two new species of the genus Atractoscion, and resurrection of the species Atractoscion atelodus (Günther 1867). (Perciformes, Sciaenidae). Zootaxa, 4306 (2): 223-237. http://dx.doi.org/10.11646/ zootaxa.4306.2.3

Spix, J. B. von and Agassiz, L. (1831). Selecta genera et species piscium quos in itinere per Brasiliam annis MDCCCXVII-MDCCCXX jussu et auspiciis Maximiliani Josephi I. Bavariae Regis augustissimi peracto collegit et pingendos curavit Dr J. B. de Spix, digessit, descripsit et observationibus anatomicis curavit Dr. L. Agassiz, praefatus est et edit itineris socios Dr. F. C. Ph. de Martius. Typis C. Wolf, Monachii. Part 2, pp. 83138.

Stark, J. (1828). Elements of natural history, adapted to the present state of the science, containing the generic characters of nearly the whole animal kingdom, and descriptions of the principal species. Vol. I. Vertebrata. Adam Black and John Stark, Edinburgh, and Longman, Rees, Orme and Green, London. vi + 527 pp.

Starks, E. C. (1906). On a collection of fishes made by P. O. Simons in Ecuador and Peru. Proceedings of the United States National Museum, 30 (468): 761-800.

Steindachner, F. (1863). Beiträge zur Kenntniss der Sciaenoiden Brasiliens und der Cyprinodonten Mejicos. Sitzungsberichte der Mathematisch-Naturwissenschaftlichen Classe der Kaiserlichen Akademie der Wissenschaften, 48: 162-185.

Steindachner, F. (1864). Ichthyologische Notizen. Sitzungsberichte der Kaiserlichen Akademie der Wissenschaften. Mathematisch-Naturwissenschaftliche Classe. Abt. 1, Mineralogie, Botanik, Zoologie, Anatomie, Geologie und Paläontologie, 49: 200-214.

Steindachner, F. (1866a). Fortsetzung der ichthyologischen Notizen. Anzeiger der Kaiserlichen Akademie der Wissenschaften, Mathematisch-Naturwissenschaftlichen Classe, 3: 19-20.

Steindachner, F. (1866b). Ichthyologische Mittheilungen. (VIII.). Verhandlungen der K.-K. zoologisch-botanischen Gesellschaft in Wien, 16: 475-484.

Steindachner, F. (1866c). Ichthyologische Notizen (III). Über einige neue Fischarten aus Südamerika. Sitzungsberichte der Mathematisch-Naturwissenschaftlichen Classe der Kaiserlichen Akademie der Wissenschaften, 53 (1. Abth.): 208-214.

Steindachner, F. (1866d). Ichthyologische Mittheilungen. (IX.) [With subtitles I-VI.]. Verhandlungen der K.-K. zoologisch-botanischen Gesellschaft in Wien, 16: 761-796.

Steindachner, F. (1866e). Über die Fische von Port Jackson in Australien. Anzeiger der Kaiserlichen Akademie der Wissenschaften, Mathematisch-Naturwissenschaftlichen Classe, 3 (7): 50-54.

Steindachner, F. (1866f). Zur Fischfauna von Port Jackson in Australien. Sitzungsberichte der Kaiserlichen Akademie der Wissenschaften. Mathematisch-Naturwissenschaftliche Classe, 53: 424-481.

Steindachner, F. (1867). Ichthyologische Notizen (VI). [With 8 subtitles.]. Sitzungsberichte der Kaiserlichen Akademie der Wissenschaften. Mathematisch-Naturwissenschaftliche Classe, 56: 307-376.

Steindachner, F. (1875a). Ichthyologische Beiträge (II). I. Die Fische von Juan Fernandez in den Sammlungen des Wiener Museums. II. Über einige neue Fischarten von der Ost- und 
Westküste Süd-Amerikas. Sitzungsberichte der Kaiserlichen Akademie der Wissenschaften. Mathematisch-Naturwissenschaftliche Classe, 71: 443-480.

Steindachner, F. (1875b). Ichthyologische Beiträge (III). Sitzungsberichte der Kaiserlichen Akademie der Wissenschaften. Mathematisch-Naturwissenschaftliche Classe, 72: 29-96.

Steindachner, F. (1876a). Ichthyologische Beiträge (V). [Subtitles i-v.]. Sitzungsberichte der Kaiserlichen Akademie der Wissenschaften. Mathematisch-Naturwissenschaftiche Classe, 74 (1. Abth.): 49-240.

Steindachner, F. (1876b). Ichthyologische Beiträge (IV) [With subtitles i-iv.]. Sitzungsberichte der Kaiserlichen Akademie der Wissenschaften. MathematischNaturwissenschaftliche Classe, 72: 551-616.

Steindachner, F. (1878a). Ichthyologische Beiträge (VII). Sitzungsberichte der Kaiserlichen Akademie der Wissenschaften. Mathematisch-Naturwissenschaftliche Classe, 78 (1. Abth.): 377-400.

Steindachner, F. (1878b). Zur Fischfauna des Magdalenen-Stromes. Anzeiger der Kaiserlichen Akademie der Wissenschaften, Wien, Mathematisch-Naturwissenschaftliche Classe, 15 (12): 88-91.

Steindachner, F. (1879a). Ichthyologische Beiträge (VIII). Sitzungsberichte der Kaiserlichen Akademie der Wissenschaften. Mathematisch-Naturwissenschaftliche Classe, 80: 119191.

Steindachner, F. (1879b). Über einige neue und seltene Fischarten aus den zoologischen Museen zu Wien, Stuttgart und Warschau. Anzeiger der Kaiserlichen Akademie der Wissenschaften, Wien, Mathematisch-Naturwissenschaftliche Classe, 16 (4): 29-34.

Steindachner, F. (1879c). Über einige neue und seltene Fisch-Arten aus den k. k. zoologischen Museum zu Wien, Stuttgart, und Warschau. Denkschriften der Kaiserlichen Akademie der Wissenschaften in Wien, MathematischNaturwissenschaftliche Classe, 41: 1-52.

Steindachner, F. (1879d). Zur Fisch-Fauna des Magdalenen-Stromes. Denkschriften der Kaiserlichen Akademie der Wissenschaften in Wien, MathematischNaturwissenschaftliche Classe, 39: 19-78.

Steindachner, F. (1902). Wissenschaftliche Ergebnisse der südarabischen Expedition in den Jahren 1898 bis 1899. Fische von Südarabien und Socotra. Anzeiger der Kaiserlichen Akademie der Wissenschaften, Wien, Mathematisch-Naturwissenschaftliche Classe, 39 (24) (art. 76): 316-318.

Steindachner, F. (1903). Fische aus Südarabien und Sokotra. Denkschriften der Kaiserlichen Akademie der Wissenschaften in Wien, Mathematisch-Naturwissenschaftliche Classe, 71: 123-168.

Steindachner, F. (1917a). Ichthyologische Beiträge (XIX). Anzeiger der Kaiserlichen Akademie der Wissenschaften, Wien, Mathematisch-Naturwissenschaftliche Classe, 54 (18): 228-229.

Steindachner, F. (1917b). Ichthyologische Beiträge (XIX). Sitzungsberichte der Kaiserlichen Akademie der Wissenschaften, Mathematisch-Naturwissenschaftlichen Klasse, 126 (Abt. 1) (8): 657-676.

Storer, D. H. (1846). A synopsis of the fishes of North America. Memoirs of the American Academy of Arts and Sciences (new series), 2 (art. 7): 253-550.

Streets, T. H. (1877). Ichthyology. In: T. H. Streets, Contributions to the natural history of the Hawaiian and Fanning islands and Lower California, made in connection with the United 
States North Pacific surveying expedition, 1873-75. Bulletin of the United States National Museum, 1 (7): 43-102.

Talwar, P. K. (1975). Blythsciaena in place of Blythia for a sciaenid genus of fishes. Newsletter of the Zoological Survey of India, 1 (2): 17.

Talwar, P. K. (1980). A new species of Atrobucca (Pisces, Sciaenidae) from the Arabian Sea. Bulletin of the Zoological Survey of India, 3 (1-2): 23-26.

Talwar, P. K. (1995). Fauna of India and the adjacent countries. Pisces. Perciformes, Sciaenidae.Zoological Survey of India, Calcutta. 144 pp.

Talwar, P. K. and Datta, A. K. (1972). A neotype and 2 topotypes for Bola chaptis Hamilton, 1822 (Pisces, Sciaenidae). Journal of Fish Biology, 4 (1): 171-172. https://doi.org/10.1111/j.1095-8649.1972.tb05663.x

Talwar, P. K. and Jhingran, A. G. (1991). Inland fishes of India and adjacent countries. Volume 2. Oxford and IBH Publishing Co., New Delhi, Bombay, Calcutta. pp, i-xxii + 543-1158 pp.

Talwar, P. K. and Joglekar, A. (1970a). On a new species of Wak Lin, 1938 (Pisces, Sciaenidae) from Hooghly Estuary. Journal of the Inland Fishery Society of India, 1: 3236.

Talwar, P. K. and Joglekar, A. (1970b). The taxonomic position of Corvina axillaris Cuvier, 1830 (Sciaenidae-Pisces). Journal of the Marine Biological Association of India, 10 (2): 361-365.

Talwar, P. K. and Sathiarajan, R. (1975). A new bathyal fish, Atrobucca trewavasae (Pisces, Sciaenidae) from the Bay of Bengal. Journal of Natural History, 9 (5): 575-580.

Talwar, P. K. (1970). A new generic name for a sciaenid fish from India. Proceedings of the Zoological Society, Calcutta, 23 (1): 67-71.

Talwar, P. K. (1971a). Taxonomic position of Corvina albida Cuvier, 1830 [Pisces, Sciaenidae]. Proceedings of the Zoological Society, Calcutta, 23 (2): 191-193.

Talwar, P. K. (1971b). The taxonomic position of Umbrina dussumieri Valenciennes, 1833 and Umbrina macroptera Bleeker, 1853 (Pisces, Sciaenidae). Journal of the Inland Fishery Society of India, 3: 22-24.

Tanaka, S. (1915). Two new species of Japanese fishes. Dobutsugaku Zasshi, Tokyo, 27 (325): 615-616. [in Japanese]

Tanaka, S. (1916). Four new species of Japanese fishes. Dobutsugaku Zasshi, Tokyo, 28 (327): 26-28. [in Japanese]

Tang, D.-S. (1937). A study of sciaenoid fishes of China. The Amoy Marine Biological Bulletin, 2 (2): 47-88.

Temminck, C. J. and Schlegel, H. (1843). Pisces, In: Siebold, P. F. de (Ed.), Fauna Japonica, sive descriptio animalium, quae in itinere per Japoniam, jussu et auspiciis superiorum, qui summum in India Batava imperium tenent suscepto annis 1823-1830 collegit, notis, observationibus et adumbrationibus illustravit Ph. Fr. de Siebold. Arnz et socios, Lugduni Batavorum. 314 pp.

Tortonese, E. (1940). Elenco dei tipi esistenti nella collezione ittiologica del Regio Museo di Torino. Bollettino del Museo di Zoologia e Anatomia Comparata di Torino (Ser. 3), 48 (111): 133-144.

Trewavas, E. (1962). A basis for classifying the Sciaenid fishes of Tropical West Africa. The Annals and Magazine of natural History (Ser. 13), 5: 167-176. 
Trewavas, E. (1963). Sciaena Linnaeus, 1758 (Pisces), proposed variation under the plenary powers of the ruling given in Opinion 93 concerning the type species. Z. N. (S.) 850. Bulletin of Zoological Nomenclature, 20: 349-360.

Trewavas, E. (1964). The sciaenid fishes with a single mental barbel. Copeia, 1964 (1): 107117. https://doi.org/10.2307/1440838

Trewavas, E. (1966). Comments: on type-species of Sciaena Linnaeus. Bulletin of Zoological Nomenclature, 23 (pt 1): 4-5.

Trewavas, E. (1971). The syntypes of the sciaenid Corvina albida Cuvier and the status of Dendrophysa hooghliensis Sinha and Rao and Nibea coibor (nec Hamilton) of Chu, Lo and Wu. Journal of Fish Biology, 3 (4): 453-461. https://doi.org/10.1111/j.10958649.1971.tb05917.x

Trewavas, E. (1973). Sciaenidae, In: Hureau, J.-C. and Monod, T. (Eds.), Check-list of the Fishes of the North-eastern Atlantic and of the Mediterranean (CLOFNAM). UNESCO, Paris. pp. 396-401.

Trewavas, E. (1977). The sciaenid fishes (croakers and drums) of the Indo-West-Pacific. The Transactions of the Zoological Society of London, 33 (4): 253-541. https://doi.org/10.1111/j.1096-3642.1977.tb00052.x

Trewavas, E. and Yazdani, G. M. (1966). Chrysochir, a new genus for the sciaenid fish Otolithus aureus Richardson, with consideration of its specific synonyms. Annals and Magazine of Natural History (Series 13), 8 (88): 249-255.

Tschudi, J. J. von (1846). Ichthyologie, In: Tschudi, J. J. von (Ed.), Untersuchungen über die Fauna Peruana. Scheitlin and Zollikofer, St. Gallen, Switzerland. pp. ii-xxx + 1-35.

Tsuboi, M., van der Bijl, W., Kopperud, B. T., Erritzoe, J., Voje, K.L., Kotrschal, A., Yopak, K. E., Collin, S. P., Iwaniuk, A. N. and Kolm, N. (2018). Breakdown of brain-body allometry and the encephalization of birds and mammals. Nature Ecology and Evolution, 2: 1495-1500. https://doi.org/10.1038/s41559-018-0632-1

Vaillant, L. L. and Bocourt, F. (1883). Études sur les poissons, In: Mission scientifique au Mexique et dans l'Amérique centrale. Recherches zoologiques. Quatrième partie. Imprimerie Nationale, Paris. pp. 121-200.

Valenciennes, A. (1843). Ichthyologie des îles Canaries, ou histoire naturelle des poissons rapportés par Webb and Berthelot, In: Webb, P. B. and Berthelot, S. (Eds.), Histoire naturelle des îles Canaries. Tome dixième. Deuxième partie. Béthune éditeur, Paris. pp. $1-109$.

Vedra, S. A., Ocampo, P. P., de Lara, A. V., Rebancos, C. M., Pacardo, E. P., Briones, N. D. (2013). Indigenous Goby population in Mandulog River system and its conservation by communities in Iligan City, Philippines. Journal of Environmental Science and Management, 16 (2): 11-18.

Victor, B. C. (1986). Duration of the planktonic larval stage of one hundred species of Pacific and Atlantic wrasses (family Labridae). Marine Biology, 90 (3): 317-326. https://doi.org/10.1007/BF00428555

Villamar, A. (1980). Totoaba, un nuevo género de la familia Sciaenidae del Golfo de California, México (Pisces, Teleostei). Anales de la Escuela Nacional de Ciencias Biológicas, México, 23 (1-4): 129-133.

Vinciguerra, D. (1926). Catalogo dei pesci raccolti a Borneo dai Sigg. Marchese G. Doria e Dott. O. Beccari negli anni 1865-67. Annali del Museo Civico di Storia Naturale di Genova (Serie 3), 10 (50): 532-628. 
Von Bonde, C. (1923). Shallow-water fishes procured by the S. S. "Pickle.". Report Fisheries and Marine Biological Survey, Union of South Africa Report, 3 (art. 1): 1-40.

Walbaum, J. J. (1792). Petri Artedi sueci genera piscium. In quibus systema totum ichthyologiae proponitur cum classibus, ordinibus, generum characteribus, specierum differentiis, observationibus plurimis. Redactis speciebus 242 ad genera 52. Ichthyologiae pars III. Ant. Ferdin. Rose, Grypeswaldiae. viii + 723 pp.

Walker, H. J. and Radford, K. W. (1992). Eastern Pacific species of the genus Umbrina (Pisces, Sciaenidae) with a description of a new species. Fishery Bulletin, 90: 574-587.

Wang, K. F. (1935). Study of the teleost fishes of the coastal region of Shangtung, II. Contributions from the Biological Laboratory of the Science Society of China. (Zoological Series), 10 (9): 393-481.

Wass, R. C. (1984). An annotated checklist of the fishes of Samoa. NOAA Technical Report of the National Marine Fishery Service, 781: i-v + 1-51.

Wheeler, A. C. (1958). The Gronovius Fish Collection, A catalogue and historical account. Bulletin of the British Museum (Natural History) Historical Series, 1: 185-249.

Whitley, G. P. (1931). New names for Australian fishes. Australian Zoologist, 6 (pt 4): 310334.

Whitley, G. P. (1950). New fish names. Proceedings of the Royal Zoological Society of New South Wales, 1948-49: 44.

Wilson, C. (1916). Some marine fishes from Colombia and Ecuador. Annals of the Carnegie Museum, 10 (1-2): 57-70.

Winterbottom, R. and Anderson, R. C. (1997). A revised checklist of the epipelagic and shore fishes of the Chagos Archipelago, Central Indian Ocean. Ichthyological Bulletin of the J. L. B. Smith Institute, (66): 1-28.

Wirtz, P., Ferreira, C. E. L., Floeter, S. R., Fricke, R., Gasparini, J. L., Iwamoto, T., Rocha, L. A., Sampaio C. L. S. and Schliewen, U. (2007). Coastal fishes of São Tomé and Principe islands, Gulf of Guinea (eastern Atlantic Ocean) - an update. Zootaxa, 1523 (1): 1-48. http://dx.doi.org/10.11646/zootaxa.1523.1.1 
Appendix: The list of institutions where the type material is located.

AFAQ: Queensland Amateur Fishing Clubs Association Inc., Australia; AMNH: American Museum of Natural History, New York, U.S.A.; AMS: Australian Museum, Sydney, New South Wales, Australia; ANSP: Academy of Natural Sciences of Drexel University, Philadelphia, Pennsylvania, U.S.A.; AZUSC: Acervo Zoológico da Universidade Santa Cecília, Santos, São Paulo, Brazil; BMNH: Natural History Museum, London, U.K.; BSKU: Kochi University, Department of Natural Science, Faculty of Science, Kochi, Japan; BSMP: Department of Agriculture, Bureau of Science, Manila, Philippines; CAS or CAS-SU: California Academy of Sciences, San Francisco, California, U.S.A.; CM: Carnegie Museum of Natural History, Pittsburgh, Pennsylvania, U.S.A.; CSIRO: Commonwealth Scientific and Industrial Research Organisation, National Collections and Marine Infrastructure, Hobart, Tasmania, Australia; DBFFEUCS: Departamento de Biología, Facultad de Ciencias, Universidad de Chile, Santiago, Chile; EBMC: Estación Biología Marina de Montemar, Instituto de Ciencias Biológicas y Químicas, Universidad de Valparaíso, Valparaíso, Chile; ENCBIPN: Escuela Nacional del Ciencias Biológicas, Instituto Politécnico Nacional; FLCR: Fundación Científica Los Roques, Caracas, Venezuela; FMNH: Division of Fishes, Department of Zoology, Field Museum of Natural History, Chicago, Illinois, U.S.A.; FMRI: Central Marine Fisheries Research Institute, Regional Centre, Mandapam Camp, India; HUMZ: Hokkaido University, Laboratory of Marine Zoology, Faculty of Fisheries, The Hokkaido University Museum, Hakodate, Hokkaido, Japan; ICNMNH: Instituto de Ciencias Naturales, Museo de Historia Natural, Facultad de Ciencias, Universidad Nacional de Colombia, Bogotá, Colombia; IMARPE: Instituto del Mar del Perú, Lima, Peru; INPA: Instituto Nacional de Pesquisas da Amazônia, Manaus, Amazonas, Brazil; INVEMAR: Instituto de Investigaciones Marinas y Costeras José Benito Vives de Andréis, Punta Betín, Santa Marta, Colombia; IRSNB: Institut Royal des Sciences Naturelles de Belgique, Bruxelles, Belgium; ISTPM: Institut Scientifique et Technique des Pêches Maritimes, Nantes, France; KFRS: Kanudi Fisheries Research Station, National Fisheries Authority, Konedobu, Papua New Guinea; KFUPM-LKR: Museum Marine Studies Section, Center of Environment and Water, King Fahd University of Petroleum and Minerals, Dhahran, Kingdom of Saudi Arabia; LACM: Natural History Museum of Los Angeles County, Los Angeles, California, U.S.A.; LBP: Laboratório de Biologia e Genética de Peixes, Departamento de Morfologia, Instituto de Biociências, Universidade Estadual Paulista "Júlio de Mesquita Filho", Campus de Botucatu, São Paulo, Brazil; LS: Linnean Society of London, London, England, United Kingdom; MACN: Museo Argentino de Ciencias Naturales "Bernardino Rivadavia", Buenos Aires, Argentina; MBUCV: Museo de Biología, Universidad Central de Venezuela, Instituto de Zoología Tropical, Caracas, Venezuela; MCP: Pontifícia Universidade Católica do Rio Grande do Sul, Museu de Ciências e Tecnologia, Rio Grande do Sul, Porto Alegre, Brasil; MCZ: Museum of Comparative Zoology, Harvard University, Ichthyology Department, Cambridge, Massachusetts, U.S.A.; MHNG: Museum d'Histoire Naturelle, Département d'Herpétologie et Ichthyologie, Ville de Genève, Genève, Switzerland; MLP: Museo de La Plata, Instituto de Limnologia, La Plata, Argentina; MNHN: Muséum National d'Histoire Naturelle, Systématique et Évolution, Laboratoire d'Ichthyologie Générale et Appliquée, Paris, France; MNHNC: Museo Nacional de Historia Natural, Zoología, Santiago, Chile; MNRJ: Museu Nacional, Departamento de Vertebrados, Setor de Ictiología, Universidade Federal do Rio de Janeiro, Rio de Janeiro, Brazil; MOBR-EDIMAR: Foundación la Salle de Science Naturales, Museo Oceanlógico Hermano Benigno Román; MAC: Ministerio de Agricultura y Cría, Instituto Nacional de Investigaciones Agricolas, Estación Experimental Apure, San Fernando de Apure, Venezuela; MPEG: Museu Paraense 'Emilio Goeldi', Zoologia, Laboratorio de Ictiologia, Belém, Pará, Brazil; MRAC: Musée Royal de l'Afrique Centrale, Tervuren, Belgium, MUFS: Department of Plant and Animal Sciences, Faculty of Agriculture, University of Miyazaki, Miyazaki, Japan; MUSM: Museo de Historia Natural de la Universidad Nacional Mayor de San Marcos, Lima, Peru; MZUSP: Universidade de São Paulo, Museu de Zoologia, São Paulo, Brazil; MZUT: Università di Torino, Dipartimento di Biologia Animale e dell'Uomo, Museo Zoologico, Torino, Italy; NMC: Canadian Museum of Nature, Ottawa, Canada; NMMBP: Pisces Collection, National Museum of Marine Biology and Aquarium, Pingtung, Taiwan; NMW: Naturhistorisches Museum, 1. Zoologische Abteilung, Fische, Wien, Austria; NSMT: National Museum of Nature and Science, Zoology Department, Division of Fishes, Tsukuba, Japan; NTM: Northern Territory Museum of Arts and 
Sciences, Ichthyology, Northern Territory Government, Darwin, Northern Territory, Australia; OMMSFC: Oman Marine Science and Fisheries Centre, Muscat, Oman; PKU: Department of Marine Biology, Pukyong National University, Busan, Korea; QM: Queensland Museum, Centre for Biodiversity, Fishes, Brisbane, Queensland, Australia; RMNH: Rijksmuseum van Natuurlijke Histoire, Leiden, Netherlands; RUSI: Rhodes University, J. L. B. Smith Institute of Ichthyology; SAIAB: South African Institute for Aquatic Biodiversity, National Research Foundation, Grahamstown, South Africa; SAM: South African Museum, Cape Town, South Africa; SFC: Laboratory of Fishes, Shanghai Fisheries University, Shanghai, China; SIO: Scripps Institution of Oceanography, Marine Vertebrate Collection, La Jolla, California, U.S.A.; SMF: Senckenberg Forschungsinstitut und Naturmuseum, Abteilung Marine Zoologie, Sektion Ichthyologie, Frankfurt am Main, Hessen, Germany; SMNS: Staatliches Museum für Naturkunde in Stuttgart, Stuttgart, BadenWürttemberg, Germany; SSCN: Museum of the Biological Laboratory, Chinese Academy of Sciences, Nanjing, China; TABL: Tropical Atlantic Biological Laboratory, National Marine Fisheries Service, Virginia Key, Miami, Florida, U.S.A.; UCLA: University of California at Los Angeles, Department of Ecology and Evolutionary Biology, Los Angeles, California; UF: University of Florida, Florida Museum of Natural History, Gainesville, Florida, U.S.A.; UMML: University of Miami, Dorothy H. and Lewis Rosenstiel School of Marine and Atmospheric Science, Division of Marine Biology and Fisheries, Miami, Florida, U.S.A.; UMMZ: University of Michigan Museum of Zoology, Ann Arbor, Michigan, U.S.A.; UMTF: Universiti Malaysia Terengganu, Malaysia; UNAM: Colección Nacional de Peces, Instituto de Biología, Facultad de Ciencias, Universidad Nacional Autónoma de México, Mexico City, Mexico; UNEFM: Universidad Nacional Experimental Francisco de Miranda, Falcón, Venezuela; UNMDP: Universidad Nacional de Mar del Plata, Funes 3350, Argentina; UPR: University of Puerto Rico Mayagüez; USNM: Smithsonian Institution National Museum of Natural History, Department of Vertebrate Zoology, Division of Fishes, Washington D.C., U.S.A.; VMM: Suffolk County Vanderbilt Museum, Centerport, New York, U.S.A.; WAM: Western Australian Museum, Department of Aquatic Zoology, Ichthyology, Perth, Western Australia, Australia; ZIN: Laboratory of Ichthyology, Zoological Institute, Russian Academy of Sciences, St. Petersburg, Russia; ZMB: Museum für Naturkunde, Leibniz-Institut für Evolutions- und Biodiversitätsforschung, Berlin, Germany; ZMUA: Zoological Museum, Department of Biology, College of Life Science, Xiamen University, Xiamen, Fujian, China; ZMUC: Københavns Universitet, Zoologisk Museum, Vertebrater, Fiskesamlingen, Copenhagen, Denmark; ZSI: Zoological Survey of India, Kolkata, India; ZUMT: Department of Zoology, University Museum, University of Tokyo, Tokyo, Japan. 